\title{
Sequential procedures for poverty gap dominance
}

\author{
Claudio Zoli* \\ Peter J. Lambert ${ }^{\dagger}$ \\ University of Bologna, Italy; \\ University of Oregon, USA. \\ University of Nottingham, UK.
}

December, 2004

\begin{abstract}
Poverty evaluations differ from welfare evaluations in one significant aspect, the existence of a threshold or reference point, the poverty line. It is therefore possible to build up normative evaluation models in which comparisons are made taking distances from this reference point and not only from the origin to be ethically relevant. This is the case in our model of poverty comparisons over heterogeneous populations, which focuses upon poverty gaps and not incomes. When poverty lines differ for the different groups in the population we show that choosing poverty gaps instead of incomes as the relevant indicator brings in normatively appealing classes of poverty indices not previously accommodated. For these indices poverty comparisons over heterogeneous populations are implemented through sequential poverty gap curves (or poverty gap distributions) dominance. These novel conditions are logically related to those suggested in Atkinson and Bourguignon (1987) for welfare comparisons, and can also be grounded firmly upon those of Bourguignon (1989). The proportion of poor individuals in the society or their average poverty gap play a role in our comparisons that was neglected in the existing poverty dominance criteria for heterogeneous populations. Various intermediate poverty dominance conditions and a generalization of the poverty gap approach are also investigated.
\end{abstract}

Keywords: poverty measurement, poverty gap, heterogeneous population, sequential dominance.

\footnotetext{
*Dipartimento di Scienze Economiche, Università di Bologna Italy and School of Economics, University of Nottingham, UK. Address: Dipartimento di Scienze Economiche, Università di Bologna, Piazza Scaravilli 2, 40126 Bologna Italy. E-mail: zoli@economia.unibo.it

${ }^{\dagger}$ Department of Economics, University of Oregon, USA. Address: Economics Department, 1285 University of Oregon, Eugene, OR 97403-1285, USA. E-mail: plambert@uoregon.edu
} 


\section{Introduction}

Consider two "poor" income units (e.g. households) with different ethically relevant non-income characteristics (e.g. needs). Now consider the two following statements:

(A) The social marginal utility of income is higher for the needier unit if experiencing the same, or a lower, income level than the less needy unit.

(B) The social marginal utility of income is higher for the needier unit if experiencing the same, or a higher, poverty gap than the less needy unit.

Both statements seem reasonable, and turn out to be equivalent if the poverty line is independent from the ethically relevant non-income characteristics, or if trivially we consider households which are homogenous in non-income characteristics. But this is not usually the case. Poverty lines are often implicitly set in order to take into account non-income characteristics; in particular, higher poverty lines are typically associated with higher levels of need. Then statements A and B turn out not to be equivalent any more. Shall we look at income levels, or at shortfalls from relevant reference points (e.g. poverty lines), in order to make normative statements (e.g. poverty evaluations)?

Our treatment of poverty comparisons over heterogeneous populations in this paper highlights some implications of following these different statements. The existing results in the poverty measurement literature within the heterogeneous setting are derived, without exception, by following approach A; we concentrate here on approach $\mathrm{B}$, making comparisons based on shortfalls from reference levels (i.e. poverty gap levels). Once this new perspective is adopted, the ensuing dominance conditions differ significantly from any of those already presented in the literature. Not least, for a fixed set of poverty lines, the proportion of poor in the society, and their average poverty gap, both play roles in our dominance comparisons. Surprisingly, this is not the case for any of the existing poverty dominance criteria in the heterogeneous case.

In order to evaluate aggregate poverty we consider additively decomposable poverty measures, defined over a population comprising subgroups that are homogeneous in terms of needs and can be ranked (in decreasing order) according to them. Individual poverty contributions are measured as the feeling of deprivation felt by income units under the poverty line when they compare their situation to the one of being non poor, that is, of having an income level at or above the poverty line. In order to take into account the differences in needs, instead of adopting cardinal equivalence scales, we follow the approach pioneered by Atkinson and Bourguignon (1987) for welfare comparisons and Atkinson (1992) for poverty comparisons. Namely, we consider distributions of non-equivalized (money) income, and appropriate poverty lines, and then identify restrictions on evaluation functions that express normative judgements about the relative impact of income changes for differences in income units' non-income characteristics. 
In the paper, we also allow some flexibility in the assumed poverty lines. In this, we adhere to the traditions of the existing poverty literature. First, in the homogeneous case, Atkinson (1987), Foster and Shorrocks (1988a,b,c), Jenkins and Lambert (1997) and Zheng (1999) all allow latitude in the poverty line to be set. Second, in the needs-based studies of Atkinson (1992), Jenkins and Lambert (1993), Chambaz and Maurin (1998) and Duclos and Makdissi (1999), poverty orderings are provided that are consistent with a variety of views about the respective poverty lines for different needs groups. But, we contend, there are three questions facing the poverty analyst, not just two, in case of social heterogeneity:

1. What is the level to assign to the poverty line $z$ ? For this purpose we can think of defining a basic level of standard of living, say $\alpha$, and allow for the setting of an income value which, given the characteristics of the income units, enables them to reach this basic level. Of course people's opinions could conflict about both the definition of standard of living and the procedure to follow in order to measure it.

2. How do poverty lines $z_{i}$ change across needs groups, call these $i=1,2, . . n$ ? This follows on from the previous point. Even if agreement is reached in respect of the minimum level of standard of living $\alpha$, then disagreement could occur when we consider the procedure to follow to assign to it an income level $z_{i}$ conditional on needs $i$. It is natural to think that higher needs require higher incomes in order to lead to similar standards of living, i.e. $z_{i} \geq z_{i+1}$ if we assume that groups are ranked in decreasing order w.r.t. needs.

3. How does deprivation, which everyone agrees should be measured in terms of shortfalls from the poverty line $z_{i}-x$ (where $x$ is the income of a poor income unit), differ across groups? Indeed, how should we compare shortfalls between income units belonging to different groups?

The emphasis on shortfalls, as in question 3, has not to our knowledge been addressed before for heterogeneous populations (though it is common enough in the homogeneous case: just consider Sen (1976) and Foster et al. (1984)). The approach we follow is very much in line with that suggested by Atkinson and Bourguignon (1987), as we explain shortly, but there is a crucial difference: our marginal utility comparisons across groups are made at fixed absolute poverty gap levels rather than fixed income levels, as there.

As a consequence of this difference in focus, the sequential poverty dominance results we obtain are different from those in Atkinson (1992), Jenkins and Lambert (1993), Chambaz and Maurin (1998), Zoli (2000) and Duclos et al. (2003). They are expressed in terms of distributions of poverty gaps and of absolute poverty gap profile (APGP) curves, introduced by Spencer and Fisher (1992), Jenkins and 
Lambert (1997) and Shorrocks $(1995,1998) .{ }^{1} \quad$ Nevertheless, the procedure is very similar in spirit to the one for checking welfare dominance, advocated by Atkinson and Bourguignon (1987) using generalized Lorenz curves. Our paper in fact provides a corresponding rationale for using APGP curves in sequential analysis.

Before moving to the analysis of our dual view, we remark upon the issue which goes to the heart of the distinction between ours and all the other approaches. Who should be the "equals" for poverty analysis? In what situation are two income units with different needs "equally poor"? Not until this question is answered, surely, can we say which income units of a needier type are socially more deserving (as in statements A and B, with which we began).

Consider briefly what would be implied for poverty analysis by the adoption of a specific set of relative equivalence scales, call them $e_{1}, e_{2}, \ldots . e_{n}$ (where, say, $e_{n}=1$, taking the least needy type $n$ as the reference type), and a minimum standard of living (equivalent income) $\alpha$. Then $z_{i}=\alpha e_{i}$ and in equivalent income space there is a common poverty line for all needs groups of $\alpha$. The normative judgement for a relative equivalence scale is that those with the same equivalent income are the equals. If amounts of real income $d x_{i}$ and $d x_{j}$ were to be given to two equals, one of type $i$ and the other of type $j>i$, the impact on poverty would be larger (smaller) for the needier income unit if $d x_{i} / z_{i}>(<) d x_{j} / z_{j}$, and would be the same if $d x_{i} / z_{i}=d x_{j} / z_{j}$. In equivalent income space, then, needs are fully taken care of by equivalizing; in that space, a common increment to the income of any member of an equals group has the same effect; there is no distinction between A and B - indeed, no call for a heterogeneous machinery.

Statements A and B both concern the relative impacts of a transfer of the same amount, but they differ in identifying the comparable sets of income units across groups. Statement A considers as comparable two units experiencing the same absolute income level, while for statement $\mathrm{B}$, the comparable units have the same absolute poverty gap. The fundamental normative judgement underpinning $B$ is that the differential poverty impact of a real income transfer should be (weakly) larger when applied to a needier household for a fixed poverty gap. If we add the assumption that the impact of a transfer on poverty deprivation decreases as income increases, for any given type, then a fortiori the differential poverty impact of a real income transfer is larger when applied to a needier household with a bigger poverty gap, too: we arrive at the full statement in B. A similar argument works with A.

Consider Figure 1, in which for the case $n=2$, an income $x_{2}$ is labelled, of a poor household belonging to the less needy group. What households in group 1 might be included in the comparability set for $x_{2}$ ? For approach $\mathrm{A}$, the comparable households have income $x_{1}^{\prime}=x_{2}$, whilst for approach $\mathrm{B}$, the comparable income is $x_{1}$, the one that yields the same poverty gap as $x_{2}$. Assuming that the impact of a

\footnotetext{
${ }^{1}$ Davidson and Duclos (2000, pp. 1439-1440) also present a procedure based on comparisons of absolute poverty gaps evaluated w.r.t. different poverty lines but considering homogeneous populations.
} 


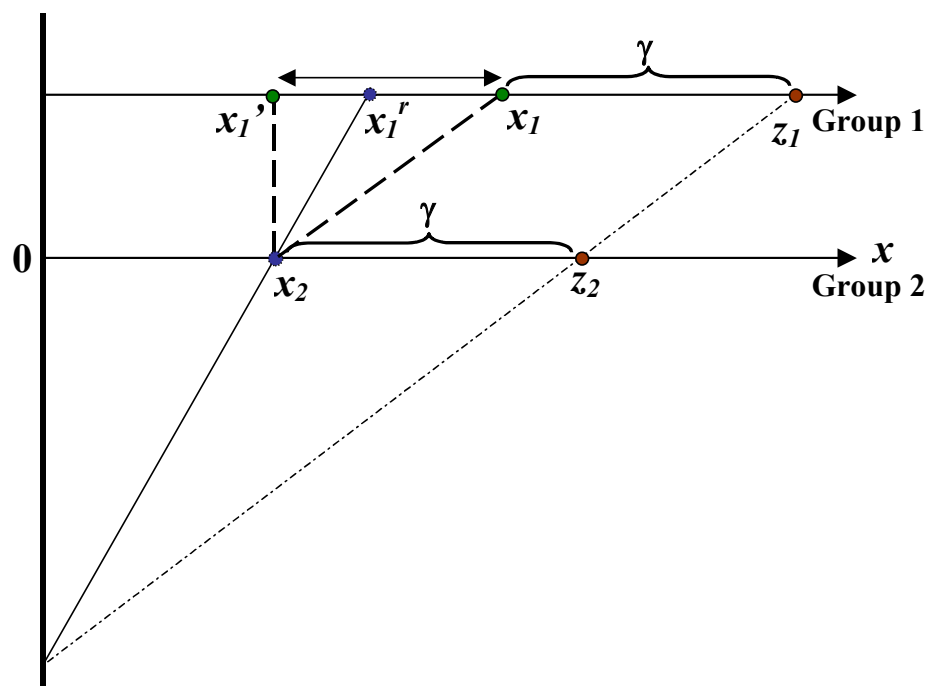

Figure 1: Comparability Sets for 2 Groups

transfer on poverty deprivation decreases as income increases, type 1 income units with less than $x_{1}^{\prime}$ (respectively, $x_{1}$ ) would a fortiori be regarded as more deserving of additional resources than $x_{2}$; those with more than $x_{1}^{\prime}$ (respectively, $x_{1}$ ), less so. One can see that identifying the "poverty equals" of $x_{2}$ as those with $x_{1}^{\prime}$ (approach A) or $x_{1}$ (approach B) presents merely two polar extremes of a whole plethora of possible value judgements about the equals; any income in the interval $\left[x_{1}^{\prime}, x_{1}\right]$ could serve; in particular, the income marked in Figure 1 as $x_{1}^{r}$ could, which has the same relative poverty gap as the original $x_{2}$. We return to this issue at the end of the paper.

The structure of the paper is as follows. In Section 2, we lay out the notation and technical preliminaries in terms of which the analysis will proceed. In Section 3, we obtain our main theorems, linking poverty dominance for defined classes of poverty indices with sequential poverty gap dominance conditions. Here we also discuss the issue of checking dominance when poverty lines $z_{i}$ vary within ranges, indicating algorithms that allow comparisons to be made for a variety of such cases. In Section 4, we make connections with existing poverty and welfare literature, showing in particular that when poverty lines are allowed to change without restrictions on upper and lower bounds, the poverty gap dominance conditions boil down to the one presented in Bourguignon (1989). We also explain here a specific sense in which our dominance criteria may be considered "more conservative" - that is, to give higher relevance to needs - than those of Atkinson (1992). Finally, in Section 5 we show how corresponding results may be obtained by extending the logic from absolute to relative poverty gaps. Section 6 concludes. 


\section{Preliminaries and notation}

We will consider poverty comparisons between income distributions over heterogeneous populations partitioned into groups of individuals homogeneous in non-income characteristics.

The discrete population is composed of $m$ income units and is partitioned into $n$ non overlapping and exhaustive population subgroups $i=1,2, . . n$ ranked in decreasing order in terms of needs. Each subgroup $i$ consists of $m_{i}>0$ income units, i.e. $\sum_{i} m_{i}=m$. The income profile of group $i$ is represented by the $m_{i}$-dimensional vector $\mathbf{x}^{i}=\left(x_{1}^{i}, x_{2}^{i}, x_{l}^{i}, . x_{m_{i}}^{i}\right)$ where $x_{l}^{i} \geq 0$ denotes the income level of income unit $l=1,2, . . m_{i}$ belonging to group $i$. The profile of the overall population is represented by the $m$-dimensional vector $\mathbf{x}=\left(\mathbf{x}^{1}, \mathbf{x}^{2}, \ldots \mathbf{x}^{i}, . . \mathbf{x}^{n}\right)$. The set of all these $m$ dimensional vectors is denoted by $X^{m}$, while $X:=\cup_{m>1} X^{m}$ denotes the union of the sets of all vectors in $X^{m}$ for all $m>1$.

Denote by $F_{i}(x)$ the cumulative income distribution of subgroup $i$ of population $F$ for $i=1,2, . . n$. The population share of individuals belonging to group $i$ is denoted by $q_{i}^{F}$. It follows that $F(x)=\sum_{i=1}^{n} q_{i}^{F} F_{i}(x)$ where $F(x)$ is the cumulative income distribution function of an income profile with support $[0,+\infty)$ and finite mean $\mu(F)=\int_{0}^{+\infty} x d F(x)$. Let $\mathcal{F}$ be the set of all such cumulative distributions. ${ }^{2}$

The poverty indices we consider are monotonic increasing transformations of the class of additively decomposable poverty indices

$$
P(F)=\sum_{i=1}^{n} q_{i}^{F} \cdot P\left(F_{i}\right)=\sum_{i=1}^{n} q_{i}^{F} \int_{0}^{z_{i}} p_{i}\left(x, z_{i}\right) d F_{i}(x) .
$$

where $P(F)\left[P\left(F_{i}\right)\right]$ measures the aggregate poverty deprivation in distribution $F$ $\left[F_{i}\right]$, while $p_{i}\left(x, z_{i}\right)$ is the individual poverty deprivation function for income units with income $x$ in group $i$ whose poverty line is $z_{i}>0$. Note that since $P(F)$ is defined over distribution functions it is within-group anonymous and population replication invariant. That is, poverty evaluation within each subgroup depends only on the income distribution and on the group's poverty line and is not affected by the identities of the income units within the subgroup i.e. is invariant w.r.t. permutations of the income profiles $\mathbf{x}^{i}=\left(x_{1}^{i}, x_{2}^{i}, \ldots x_{l}^{i}, \ldots x_{m_{i}}^{i}\right)$. Furthermore the poverty evaluation is invariant w.r.t. replications of the income profile $\mathbf{x}$, where to each individual is associated a finite number of "clones".

Let $F_{i}^{-1}(p):=\inf \left\{x: F_{i}(x) \geq p\right\}$ with $p \in[0,1]$ be the left continuous inverse of $F_{i}(x)$ showing the income of an individual at the $p$ population quantile of the distribution of group $i$. Correspondingly $F^{-1}(p)$ will represent the left continuous

\footnotetext{
${ }^{2}$ The results we will present can be extended [with a slight change in notation] to income distribution domains including bounded negative incomes, i.e. considering income profiles with support $[\underline{x},+\infty)$ for $\underline{x} \leq 0$.
} 
inverse of $F(x)$. Let $\gamma^{F}(p, z)$ be the "absolute poverty gap profile" of the censored distribution $F$ evaluated at the $p$ quantile of the income distribution of the total population, for a given poverty line $z$. That is

$$
\gamma^{F}(p, z)= \begin{cases}z-F^{-1}(p) & \text { if } F^{-1}(p) \leq z \\ 0 & \text { otherwise }\end{cases}
$$

Note that $\gamma^{F}(p, z)$ is non-increasing in $p$ and $\gamma^{F}(p, z)=0$ if $p \geq F(z)$. For a discrete distribution $\gamma^{F}(j / m, z)=z-x_{j}^{*}$ where $m$ is the size of the whole population, and $x_{j}^{*}$ is a generic element of the vector $\mathbf{x}^{*}$ which ranks incomes, censored at level $z$, in decreasing order (i.e. $x_{j}^{*}=x_{j}$ if $z>x_{j}$ and $x_{i}^{*}=z$ if $z \leq x_{i}$, so that $x_{j}^{*} \geq x_{j-1}^{*}$ ). If we denote by $\pi$ the proportion of poor individuals in the population (i.e. $\pi=F(z)$ ) it will follow that $\gamma^{F}(j / m, z)=0$ for all $j / m>\pi$.

The absolute poverty gap profile (APGP) curve is the cumulated curve of the absolute poverty gaps:

\section{Definition 1 (APGP Curve)}

$$
\mathcal{P} \mathcal{G}_{F}(p, z)=\int_{0}^{p} \gamma^{F}(q, z) d q
$$

This curve (in its relative formulation, taking into account relative poverty gaps) has been introduced in poverty analysis by Spencer and Fisher (1992), Jenkins and Lambert (1997) and Shorrocks $(1995,1998)$.

Note that $\mathcal{P G}_{F}(t, z)=\mathcal{P} \mathcal{G}_{F}(F(z), z)=H(F, z) I(F, z)$ for all $t \geq F(z)$, where $H(F, z)$ is the headcount ratio of distribution $F$ and $I(F, z)$ is its average absolute income gap.

In order to compare our results to those existing in the literature we present here the classical Sequential Poverty Dominance (SPD) conditions suggested in Atkinson (1992), Jenkins and Lambert (1993) and Chambaz and Maurin (1998). Given the ranking of needs between groups, we suppose that the vector $\mathbf{z} \in \mathbb{R}_{++}^{n}$ of poverty lines is ranked in non-increasing order, i.e. $z_{i} \geq z_{i+1}$. The set of all these vectors of poverty lines is denoted with $Z^{n}$. We will write $F \succcurlyeq_{S P D(j)[\mathbf{z}]} G$ to denote that distribution $F$ dominates $G$ according to the (SPD) condition of order $j=1,2$. These comparisons are made for a given vector of ordered poverty lines $\mathbf{z} \in Z^{n}$. The definition for SPD is presented here for the general case where marginal distributions of needs may differ.

Definition 2 (Sequential Poverty Dominance ) For $\mathbf{z} \in Z^{n}$ and $F, G \in \mathcal{F}$ :

(1) $F \succcurlyeq_{S P D(1)[\mathbf{z}]} G \Longleftrightarrow \sum_{i=1}^{k}\left[q_{i}^{F} F_{i}(x)-q_{i}^{G} G_{i}(x)\right] \leq 0$ for all $x \leq z_{k}$, for all $k=1,2, \ldots n$,

(2) $F \succcurlyeq_{S P D(2)[\mathbf{z}]} G \Longleftrightarrow \sum_{i=1}^{k} \int_{0}^{x}\left[q_{i}^{F} F_{i}(t)-q_{i}^{G} G_{i}(t)\right] d t \leq 0$ for all $x \leq z_{k}$, for all $k=1,2, \ldots n$. 
These are sequential stochastic dominance conditions of the type suggested in Atkinson and Bourguignon (1987) where comparisons at each stage $k$ are restricted to income levels below the poverty line $z_{k}$. For example, take the case $k=2$, poor individuals in the neediest group with incomes within the range $\left(z_{2}, z_{1}\right]$ are not considered. They are, of course, considered at the first stage of comparison. That is, only individuals in group $j$ with sufficiently low income are considered at the stage $k>j$.

According to the new criterion we shall introduce, by contrast, at each stage $k$ of comparison all of the poor individuals in groups $j \leq k$ play a role. The following example will illustrate a situation where SPD conditions lead to a counter-intuitive result.

Example 1 Consider the income profiles $\mathbf{x}$ and $\mathbf{y}$, each defined over the same population comprising two subgroups with different needs. Let the respective distribution functions be $F$ and $G$, and let the incomes be as follows (denoting subgroups by superscripts 1 and 2):

$$
\begin{aligned}
& \mathbf{x}^{1}=(1,3,6), \mathbf{x}^{2}=(0,4,4) \\
& \mathbf{y}^{1}=(0,2,4), \mathbf{y}^{2}=(1,4,6) .
\end{aligned}
$$

Further suppose that the poverty lines are $z_{1}=7$ and $z_{2}=5$. Note in respect of the first subgroup that $(1,3,6)$ first order stochastically dominates $(0,2,4)$. Note also that, merging all the individuals in subgroups 1 and 2 with incomes at most $5=z_{2}$, $(0,1,3,4,4)$ first order stochastically dominates $(0,1,2,4,4)$. From these two observations it follows that $F \succcurlyeq_{S P D(1)[7,5]} G$. However $5 / 6$ of the population in $\mathbf{y}$ is poor while in $\mathbf{x}$ all individuals are poor.

From the example is clear that although $\mathbf{x}$ dominates $\mathbf{y}$ according to $S P D(1)$ it may include also a proportionally larger number of poor individuals. The head-count ratio is the most common crude poverty indicator. It is surprising that the partial order $S P D(1)$ devised to accommodate a variety of views about poverty without imposing any concern for "poverty-intensity" and "poverty-inequality" turns out not to be consistent with the headcount ratio. ${ }^{3}$

The following example provides a similar result where $\operatorname{SPD}(2)$ conflicts with the average poverty gap indicator evaluated over the entire population.

\footnotetext{
${ }^{3}$ The inconsistency between $\operatorname{SPD}(1)$ and the head-count ratio has been pointed out by Atkinson (1992, p.8) (see also Duclos and Makdissi, 1999). According to Atkinson the head-count ratio is ruled out from the set of poverty measures considered by the assumption of continuity of $p_{i}\left(x, z_{i}\right)$ at $x=z_{i}$, and therefore it should not play a role in our dominance conditions. We will show that even if $p_{i}\left(x, z_{i}\right)$ is continuous at $x=z_{i}$, the headcount ratio (or its version considering the proportion of strictly poor individuals) will play a role in the dominance conditions. The result is due to the change in perspective to comparisons made at poverty gap levels instead of income levels.
} 
Example 2 Following the same notation introduced in the previous example, consider the income profiles $\mathbf{x}$ and $\mathbf{y}$ (with distribution functions respectively $F$, and $G$ ) where:

$$
\begin{aligned}
& \mathbf{x}^{1}=(1,2,6), \mathbf{x}^{2}=(0,4,4) \\
& \mathbf{y}^{1}=(0,2,4), \mathbf{y}^{2}=(1,4,8)
\end{aligned}
$$

Suppose that the subgroups poverty lines are $z_{1}=9$ and $z_{2}=7$. Then $F \succcurlyeq_{S P D(2)[9,7]} G$ but the average poverty gap in $\mathbf{y}$ is $5=(21+9) / 6$ while in $\mathbf{x}$ it is $31 / 6=(18+13) / 6$ which is larger.

\section{The main results}

As a starting point we assume that it is possible to specify a profile of poverty lines $\mathbf{z}=\left(z_{1}, z_{2}, . . z_{i}, . . z_{n}\right) \in Z^{n}$ ranked in non-increasing order such that poverty deprivation is eliminated for all individuals with income levels not below the poverty line and for higher level of needs not less income is required to achieve a minimum standard of living.

For a given poverty line $z_{i}$ and income level $x$ the individual deprivation function $p_{i}\left(x, z_{i}\right)$ can be expressed in terms of absolute poverty gaps $z_{i}-x^{*}$, where $x^{*}$ denotes income censored at level $z_{i}$. That is

$$
p_{i}\left(x, z_{i}\right)= \begin{cases}0 & \text { if } z_{i}<x \\ u_{i}\left(z_{i}-x\right) & \text { if } z_{i} \geq x\end{cases}
$$

where $u_{i}($.$) is such that u_{i}(0)=0$ for all $i$. For a poverty gap $\gamma$ such that

$$
\gamma= \begin{cases}0 & \text { if } z_{i}<x \\ z_{i}-x & \text { if } z_{i} \geq x\end{cases}
$$

the function $u_{i}(\gamma)$ may depend also on $z_{i}$. If $u_{i}(\gamma)$ is independent from $z_{i}$, this generates the class of additively decomposable poverty indices expressed in terms of poverty gaps. This is a subset of the class of poverty indices considered in Spencer and Fisher (1992), and Chakravarty $(1983,1983 a)$. Indices included in this group are those of Foster et al. (1984), obtained for $u(z-x)=(z-x)^{\alpha}$ when homogeneous populations are considered and poverty is evaluated in absolute terms. ${ }^{4}$ The headcount ratio, i.e. the proportion of poor individuals in the society, is not included in the class of indices considered. We would require $u_{i}(0)=1$ for all $i$ so that for this index $p_{i}\left(x, z_{i}\right)$ is discontinuous at $x=z_{i}$.

\footnotetext{
${ }^{4}$ An heterogeneous populations class of poverty indices generalizing the FGT class can be obtained letting $u_{i}\left(z_{i}-x\right)=\left(z_{i}-x\right)^{\alpha_{i}}$ where the coefficients $\alpha_{i}$ may depend on the groups.
} 


\subsection{Characterization of Poverty Indices}

Policy makers may have different opinions about $\mathbf{z} \in Z^{n}$ (see points 1 and 2 in the introduction) and/or the function $u_{i}($.$) (see point 3). We will first consider a fixed$ vector $\mathbf{z}$ and derive dominance conditions based on reasonable assumptions on $u_{i}(.){ }^{5}$ Then, we will extend the analysis in order to allow for possible changes in $\mathbf{z}$, and we will show that if the changes in poverty lines satisfy some restrictive assumptions it is possible to implement simplified procedures that allow to extend the poverty comparisons for a fixed poverty lines profile to the case where poverty lines may change.

The following assumptions make clear the normative value judgements we apply in order to allow partial comparisons of deprivation felt by individuals belonging to different groups, making use of ordinal information on needs. We consider functions $u_{i}(\gamma)$ that are continuous and twice differentiable.

Property $\mathbf{A} u_{i}(\gamma) \geq 0$ for all $\gamma>0$, and $u_{i}(0)=0$, for all $i=1,2, . . n$.

This property requires that perceiving a positive shortfall $\gamma$ w.r.t. the poverty line is not beneficial for an individual's well-being, thereby inducing a positive level of individual poverty. The ensuing properties introduce the assumptions imposed on between-group comparisons of changes in poverty gaps.

Property A1 $u_{i}^{\prime}(\gamma) \geq u_{i+1}^{\prime}(\gamma) \geq 0$ for all $\gamma>0$, and all $i=1,2, . . n-1$.

Changes in income show a greater effect on the well-being of an individual with a given poverty gap the higher is the level of needs. The needier is an individual, the higher is the positive impact on his/her deprivation of a loss in income for a fixed level of poverty gap.

We introduce two axioms here, in respect of a poverty measure $P($.$) , defined as in$ (1), which correspond to property A1. When between groups comparisons are taken into account we impose the restriction of identical marginal distributions of needs in order to separate the issue of group importance (in terms of needs) from the one of group size.

Axiom 1 (WM Weak Monotonicity) For all distributions $F, G \in \mathcal{F}$ such that $F$ is obtained from $G$ by reducing the income of a poor individual, $P(F) \geq P(G)$.

\footnotetext{
${ }^{5}$ The standard assumption on $u_{i}($.$) when poverty is evaluated over homogeneous populations is$ that $u_{i}($.$) is non-decreasing and convex in the poverty gap level, equivalently poverty satisfies re-$ spectively the weak monotonicity property requiring that a decrease in income of a poor individual should not reduce poverty, and the weak Principle of Transfers requiring that as a result of a progressive income transfer poverty should not increase. That is, for $u_{i}(\gamma)$ assumed twice differentiable, $u_{i}^{\prime}(\gamma) \geq 0$, and $u_{i}^{\prime \prime}(\gamma) \geq 0$ for all $\gamma \geq 0$, all $z_{i}>0$, and all $i=1,2, . . n$.
} 
Axiom 2 (BGP Between Group Priority) For all $\gamma>0, \delta>0, j>i$ and all distributions $F, F^{\prime}, G \in \mathcal{F}$ where $q_{i}^{G}=q_{j}^{G}=q$, such that $F$ (respectively $F^{\prime}$ ) is obtained from $G$ through a reduction $\delta>0$ of the income of an individual at poverty gap $\gamma>0$ in group $i$ (resp. $j$ ) $P(F) \geq P\left(F^{\prime}\right)$.

$P($.$) satisfies Axioms \mathrm{WM}$ and BGP if and only if $u_{i}(\gamma)$ satisfies property A1. See the Appendix for the demonstration of this and all subsequent mathematical assertions, which shall be suppressed from the main text for ease of presentation.

The next property allows to compare, between individuals in different groups, the marginal effect of transfers at fixed poverty gap levels.

Property A2 $u_{i}^{\prime \prime}(\gamma) \geq u_{i+1}^{\prime \prime}(\gamma) \geq 0$ for all $\gamma>0$, and all $i=1,2, . . n-1$.

The differential impact of increases in poverty gaps between individuals with different needs increases as their shortfalls w.r.t. the poverty line increase. Consider a progressive transfer of $\delta>0$ occurring between individuals in group $i$ where the donor experiences the poverty gap $\gamma$ and the receiver has poverty gap $\gamma+\varepsilon$ where $\varepsilon>0$, and a similar transfer involving individuals in group $j>i$. Requiring that both transfers have a non increasing effect on the groups' poverty and that the poverty reduction effect cannot be lower for the transfer occurring in the needier group we get A2. As for A1, we can couch these properties axiomatically.

Axiom 3 (WPT: Weak Principle of Transfers) For all distributions $F, G \in \mathcal{F}$ such that $F$ is obtained from $G$ through a regressive transfer involving poor income units, $P(F) \geq P(G)$.

Axiom 4 (BGTP: Between Group Transfer Priority) For all $\gamma>0, \varepsilon>0$, $\delta>0, j>i$ and all distributions $F, F^{\prime}, G \in \mathcal{F}$ where $q_{i}^{G}=q_{j}^{G}=q$, such that $F$ (resp. $\left.F^{\prime}\right)$ is obtained from $G$ through a regressive transfer $\delta>0$ from an individual at poverty gap $\gamma+\varepsilon>0$ to an individual at poverty gap $\gamma>0$ both in group $i$ (resp. j) $P(F) \geq P\left(F^{\prime}\right)$.

$P($.$) satisfies Axiom WPT and BGTP if and only if u_{i}(\gamma)$ satisfies property A2. ${ }^{6}$

Note that properties A and A1 imply that $u_{i}(\gamma) \geq u_{i+1}(\gamma)$ for all $\gamma \geq 0$. Moreover, if we add to these properties the requirement of WPT, i.e. if $u_{i}^{\prime \prime}(\gamma) \geq 0$, then if poverty lines are set such that $z_{i} \geq z_{i+1}$ it will follow that for all $i=1,2, . . n-1$ and all $x \in \mathbb{R}, p_{i}^{\prime}\left(x, z_{i}\right) \leq p_{i+1}^{\prime}\left(x, z_{i+1}\right) \leq 0$ where $p_{i}^{\prime}\left(x, z_{i}\right)$ denotes the partial derivative of the individual poverty deprivation function $p_{i}\left(x, z_{i}\right)$ w.r.t. $x$.

As a result dominance for all poverty indices satisfying A, A1 and WPT will be implied by dominance for all indices satisfying $p_{i}\left(x, z_{i}\right) \geq 0$ if $x \leq z_{i}$ otherwise $p_{i}\left(x, z_{i}\right)=0$ and $p_{i}^{\prime}\left(x, z_{i}\right) \leq p_{i+1}^{\prime}\left(x, z_{i+1}\right) \leq 0$ for all $x \in \mathbb{R}$. This class of indices is

\footnotetext{
${ }^{6}$ The class of indices obtained letting $u_{i}\left(z_{i}-x\right)=\left(z_{i}-x+1\right)^{\alpha_{i}}-1$ where $\alpha_{i} \geq \alpha_{i+1} \geq 0$ satisfy $\mathrm{A}$ and $\mathrm{A} 1$. If in addition is required $\alpha_{i} \geq \alpha_{i+1} \geq 1$ then also A2 is satisfied.
} 
considered in Atkinson (1992). As will emerge, it is larger than the class of those satisfying A, A1 and WPT in that it is possible when $z_{i}>z_{i+1}$ to construct individual deprivation functions that satisfy the previous condition but not A1 (see Appendix). As a result the dominance conditions SPD(1) derived in Atkinson (1992), Jenkins and Lambert (1993) and Chambaz and Maurin (1998) will imply those obtained for poverty indices satisfying A, A1 and WPT and therefore also those satisfying A, A1 and A2.

\subsection{Aggregate Poverty Comparisons}

The poverty deprivation content of distribution $F$ is $P(F)$ where

$$
P(F)=\sum_{i=1}^{n} q_{i}^{F} \int_{0}^{z_{i}} u_{i}\left(z_{i}-x\right) d F_{i}(x)
$$

(compare (1)). This measure can be interpreted as the average well-being shortfall from a minimum standard of living. The poverty deprivation differential between income distributions $F$ and $G$ is given by $\Delta P$ :

$$
\begin{aligned}
\Delta P & =\sum_{i=1}^{n} q_{i}^{F} \int_{0}^{z_{i}} u_{i}\left(z_{i}-x\right) d F_{i}(x)-\sum_{i=1}^{n} q_{i}^{G} \int_{0}^{z_{i}} u_{i}\left(z_{i}-x\right) d G_{i}(x) . \\
& =\sum_{i=1}^{n} \int_{0}^{z_{i}} u_{i}\left(z_{i}-x\right)\left[q_{i}^{F} d F_{i}(x)-q_{i}^{G} d G_{i}(x)\right] .
\end{aligned}
$$

We can now set out the first proposition, specifying the conditions for first degree sequential poverty deprivation dominance. This is a reference-based dominance concept. We use this terminology throughout, whenever the conditions we derive resemble standard stochastic dominance criteria but instead of checking dominance at each income level for all groups, they check for dominance at income levels associated with the same poverty gap in each group. When comparisons are made over subgroups under the assumption of the same poverty line in each group, the conditions are the same as for standard stochastic dominance, but when poverty lines differ the conditions are modified: instead of comparing subgroup distributions at each income level we compare distributions $F_{i}\left(z_{i}-\gamma\right)$ at each poverty gap level $\gamma \geq 0$. If marginal distributions of needs differ between income distributions then the distribution functions considered will be weighted according to the population share of the group. Formally, let

$$
\phi_{i}^{F}\left(\gamma, z_{i}\right):=\left\{\begin{array}{cl}
q_{i}^{F} \cdot F_{i}\left(z_{i}-\gamma\right) & \text { if } z_{i} \geq \gamma \\
0 & \text { if } z_{i}<\gamma
\end{array}\right.
$$

denote the proportion, over the entire population in $F$, of individuals belonging to group $i$ whose poverty gap is not-lower than $\gamma$, and similarly for $G$. Furthermore let $\gamma^{*}$ denote the highest poverty gap in any subgroup. Given that incomes are non-negative and $z_{i} \geq z_{i+1}>0$, it follows that $\gamma^{*} \in\left(0, z_{1}\right]$. 
Definition 3 (RBD: Reference Based SPD) For $\mathbf{z} \in Z^{n}$, and $F, G \in \mathcal{F}$ :

(i) $F \succcurlyeq_{R B D(1)[\mathbf{z}]} G \Longleftrightarrow \sum_{i=1}^{k}\left[\phi_{i}^{F}\left(\gamma, z_{j}\right)-\phi_{i}^{G}\left(\gamma, z_{j}\right)\right] \leq 0$ for all $\gamma>0$, all $k=$ $1,2, \ldots n$,

(ii) $F \succcurlyeq_{R B D(2)[\mathbf{z}]} G \Longleftrightarrow \sum_{i=1}^{k}\left(\int_{\gamma}^{\gamma^{*}}\left[\phi_{i}^{F}\left(t, z_{i}\right)-\phi_{i}^{G}\left(t, z_{i}\right)\right] d t\right) \leq 0$ for all $\gamma>0$, all $k=1,2, \ldots n$.

The condition $R B D(1)$ is a first-degree stochastic dominance condition where comparisons are made at levels of poverty gaps. As long as we consider only the first group $(k=1)$ the dominance condition corresponds to $q_{1}^{F} F_{1}(x) \leq q_{1}^{G} G_{1}(x)$ for all $x \in\left[0, z_{1}\right)$. Therefore the first stage of the "reference-based" dominance coincides with the standard first degree Sequential Poverty Dominance condition. Once we consider multiple groups the equivalence with $\operatorname{SPD}(1)$ is lost. The reason is that comparisons are made at the same level of poverty gap between different groups and not at income levels. Only if $z_{i}=z$ for all $i$ does the new reference-based criterion correspond to the standard first degree sequential poverty dominance condition.

A practical way of implementing the set of dominance comparisons is to consider as reference point the poverty line $z_{1}$ which is the highest, then shift to the right the distribution functions of each group $i$ by $\theta_{i}=z_{1}-z_{i}$, such that all the poverty lines now coincide, then apply the first order sequential dominance condition to the new shifted distributions. In the Appendix, we provide an example to show a set of comparisons where first degree reference-based poverty dominance is satisfied but first degree standard sequential poverty dominance is not.

We now consider the second order of reference-based sequential poverty dominance, which we shall link with dominance in terms of absolute poverty gap profile (APGP) curves in the next sub-section.

Consider for simplicity of exposition the case of two groups. The dominance condition becomes: $\int_{\gamma}^{\gamma^{*}}\left[\phi_{1}^{F}\left(t, z_{1}\right)-\phi_{1}^{G}\left(t, z_{1}\right)\right] d t \leq 0$ for all $\gamma>0$ at the first stage, and $\int_{\gamma}^{\gamma^{*}}\left[\phi_{1}^{F}\left(t, z_{1}\right)+\phi_{2}^{F}\left(t, z_{2}\right)-\phi_{1}^{G}\left(t, z_{1}\right)-\phi_{2}^{G}\left(t, z_{2}\right)\right] d t \leq 0$ for all $\gamma>0$ for the whole population. Note that $\phi_{1}^{F}\left(t, z_{1}\right)+\phi_{2}^{F}\left(t, z_{2}\right)$ gives the proportion of individuals (in the whole population) with income such that their poverty gap is at least $t$, irrespective of their group.

In general $\sum_{i=1}^{k} \phi_{i}^{F}\left(\gamma, z_{i}\right)$ provides the distribution of the poverty gaps in the first $k$ groups, and the condition $\sum_{i=1}^{k}\left(\int_{\gamma}^{\gamma^{*}}\left[\phi_{i}^{F}\left(t, z_{i}\right)-\phi_{i}^{G}\left(t, z_{i}\right)\right] d t\right) \leq 0$ for all $\gamma>0$ can be considered as a second degree stochastic dominance condition over homogeneous populations, obtained by shifting incomes in order that poverty lines for all groups coincide and then making comparisons at all shifted income levels that by construction will be associated with the same absolute poverty gap in each subgroup. For a graphical illustration see Example 4 in the Appendix.

The following proposition will highlight the link between the RBD conditions and the poverty dominance conditions associated with the poverty indices $P($.$) .$ 
Proposition 1 For a fixed $\mathbf{z} \in Z^{n}$,

(i) $P(F) \leq P(G)$ for all $u_{i}$ satisfying $A$ and $A 1 \Longleftrightarrow F \succcurlyeq_{R B D(1)[\mathbf{z}]} G$.

(ii) $P(F) \leq P(G)$ for all $u_{i}$ satisfying $A, A 1$ and $A \mathscr{2} \Longleftrightarrow F \succcurlyeq_{R B D(2)[\mathbf{z}]} G$.

It is important to point out a minor difference between $\operatorname{RBD}(1)$ and $\operatorname{SPD}(1)$. The $\operatorname{RBD}(1)$ condition is required to hold only for $\gamma>0$, that is individuals exactly at the poverty line are not considered. Therefore comparisons of head-count ratios are not required, instead for $\gamma \rightarrow 0 \mathrm{RBD}(1)$ requires that $\sum_{i=1}^{k} q_{i}^{F} \cdot H^{-}\left(F_{i}, z_{i}\right) \leq$ $\sum_{i=1}^{k} q_{i}^{G} \cdot H^{-}\left(G_{i}, z_{i}\right)$ where $H^{-}$denotes the head-count ratio evaluated making use of the "strict" definition of poverty, i.e. considering as "poor" only incomes strictly below the poverty line. This modification is due to the fact that we consider only discrete income distributions. It is possible to extend the results in Proposition 1 (part i) to comparisons between continuous distributions, in this case $H^{-}$and $H$ will coincide. $^{7}$

\subsection{Sequential Poverty Gap Dominance Conditions}

As shown in Foster and Shorrocks (1988a,b,c), Spencer and Fisher (1992), Jenkins and Lambert (1997) and Shorrocks $(1995,1998)$ when poverty comparisons are made over homogeneous populations first and second order stochastic dominance for censored income distributions are equivalent to respectively rank dominance of absolute poverty gaps distributions and APGP curve dominance. An analogous relation exists between the reference-based sequential poverty conditions in the previous propositions and appropriate sequential dominance conditions for heterogeneous populations expressed in terms of poverty gaps. We introduce these Sequential Poverty Gap Dominance (SPGD) conditions here and prove the equivalence with the reference-based poverty dominance conditions characterized in Proposition 1.

Let $\mathbf{z}^{k}=\left(z_{1}, \ldots z_{i}, . . z_{k}\right)$ be the vector of poverty lines associated with the first $k$ groups, and let $z^{*}:=\max \left\{z_{1}, z_{2}, \ldots, z_{i}, . ., z_{n}\right\}$; given that poverty lines are ranked in decreasing order $z^{*}=z_{1}$. If we let

$$
F^{(k)}\left(t, \mathbf{z}^{k}\right):=\frac{\sum_{i=1}^{k} q_{i}^{F} F_{i}\left(t-z^{*}+z_{i}\right)}{\sum_{i=1}^{k} q_{i}^{F}} \text { for all } t \in\left[0, z^{*}\right]
$$

where $F_{i}(y):=0$ if $y<0$, we obtain the distribution that, for any "poor" income level $t$ in the group with the highest poverty line, identifies the proportion of income units in the first $k$ subgroups that experience an income gap of at least $z^{*}-t$.

The poverty gap of the individual ranked at the $p^{\text {th }}$ population quantile of the income distribution $F^{(k)}$, when the poverty lines are set at $\mathbf{z}^{k}$, can be written as

$$
\gamma^{F^{(k)}}\left(p, \mathbf{z}^{k}\right)=\left\{\begin{array}{cc}
z^{*}-F^{(k)-1}\left(p, \mathbf{z}^{k}\right) & \text { if } F^{(k)-1}\left(p, \mathbf{z}^{k}\right) \leq z^{*} \\
0 & \text { otherwise }
\end{array}\right.
$$

\footnotetext{
${ }^{7}$ If (3) is modified s.t. $u_{i}(0)=a_{i} \geq 0$ if $x=z_{i}$ in order to quantify the "stigma effect" of being poor, and furthermore it is assumed that $a_{i} \geq a_{i+1}$ for all $i=1,2, . . n-1$, then the dominance condition in Proposition 1 (part 1) has to consider also $\gamma=0$.
} 
where $F^{(k)-1}\left(p, \mathbf{z}^{k}\right)$ is the left continuous inverse function of $F^{(k)}\left(t, \mathbf{z}^{k}\right)$. It follows that $\mathcal{P} \mathcal{G}_{F}^{k}\left(p, \mathbf{z}^{k}\right)=\int_{0}^{p} \gamma^{F^{(k)}}\left(t, \mathbf{z}^{k}\right) d t$ where $\mathcal{P} \mathcal{G}_{F}^{k}\left(p, \mathbf{z}^{k}\right)$ denotes the absolute poverty gap curve of the first $k$ groups of population $F$, where poverty gaps in each group $i$ are evaluated w.r.t. the group poverty line $z_{i}$. Note that $\mathcal{P} \mathcal{G}_{F}^{k}(1)=\sum_{i=1}^{k} q_{i}^{F} H\left(F_{i}, z_{i}\right)$. $I\left(F_{i}, z_{i}\right) / \sum_{i=1}^{k} q_{i}^{F}$ is the average absolute income gap [averaged w.r.t. the entire population] where the first $k$ groups are merged and individuals in each group are considered poor if their income is not above the group poverty line.

We present new poverty dominance conditions for heterogeneous populations expressed in terms of the sequential absolute poverty-gap distributions $\gamma^{F^{(k)}}\left(p, \mathbf{z}^{k}\right)$. These conditions correspond to rank-dominance of the poverty gap distributions $\gamma^{F^{(k)}}\left(p, \mathbf{z}^{k}\right)$ [first order of dominance] and to sequential dominance in terms of the poverty-gap curves $\mathcal{P} \mathcal{G}_{F}^{k}\left(p, \mathbf{z}^{k}\right)$ [second order of dominance]. We will write $F \succcurlyeq_{S P G D(j)}$ $G$ to denote that distribution $F$ dominates $G$ according to the Sequential Poverty Gap Dominance (SPGD) condition of order $j=1,2$. We first state the conditions under the simplifying assumption of common marginal distributions of needs.

Definition 4 (SPGD: Sequential Poverty Gap Dominance ) For a fixed $\mathbf{z} \in$ $Z^{n}$ and $F, G \in \mathcal{F}$ s.t. $q_{i}^{F}=q_{i}^{G}$ for all $i$ :

(i) $F \succcurlyeq_{S P G D(1)[\mathbf{z}]} G \Longleftrightarrow \gamma^{F^{(k)}}\left(p, \mathbf{z}^{k}\right) \leq \gamma^{G^{(k)}}\left(p, \mathbf{z}^{k}\right)$ for all $p \in[0,1]$, for all $k=1,2, \ldots n$,

(ii) $F \succcurlyeq_{S P G D(2)[\mathbf{z}]} G \Longleftrightarrow \mathcal{P} \mathcal{G}_{F}^{k}\left(p, \mathbf{z}^{k}\right)=\int_{0}^{p} \gamma^{F^{(k)}}\left(p, \mathbf{z}^{k}\right) \leq \int_{0}^{p} \gamma^{G^{(k)}}\left(p, \mathbf{z}^{k}\right)=\mathcal{P} \mathcal{G}_{G}^{k}\left(p, \mathbf{z}^{k}\right)$, for all $p \in[0,1]$, for all $k=1,2, \ldots n$.

In the next two propositions we show the equivalence between the reference-based dominance conditions and the general version of the SPGD conditions. Let $Q_{k}^{F}=$ $\sum_{i=1}^{k} q_{i}^{F}$ denote the population share of the first $k$ groups.

Proposition 2 Let $k=1,2, . . n$. The following conditions are equivalent:

(i) $F \succcurlyeq_{R B D(1)[\mathbf{z}]} G$

(ii) (a) $\gamma^{F^{(k)}}\left(p / Q_{k}^{F}, \mathbf{z}^{k}\right) \leq \gamma^{G^{(k)}}\left(p / Q_{k}^{G}, \mathbf{z}^{k}\right)$ for all $p \in\left[0, \min \left\{Q_{k}^{F}, Q_{k}^{G}\right\}\right]$ and (b) $\sum_{i=1}^{k} q_{i}^{F} \cdot H\left(F_{i}, z_{i}\right) \leq Q_{k}^{G}$.

Condition (iia) adjusts the procedure for poverty gaps rank dominance in order to take into account that sequences of subgroups may cover different population shares, ensuring that the relative ranks considered are those evaluated with respect to the entire population. Condition (iib) simply requires that the proportion of poor individuals in the $k$ neediest groups of population $F$ is lower than the proportion of individuals $\mathrm{i}$ the same groups of population $G$. Note that when all groups are considered condition (iia) implies (iib) and requires rank dominance of the whole population distributions of poverty-gaps. Note also that when $Q_{k}^{F}=Q_{k}^{G}$ conditions (iia) and (iib) boil down into those for SPGD(1) evaluated at stage $k$.

The dominance condition in Proposition 1 (part ii) can be equivalently expressed making use of the poverty gap curves $\mathcal{P} \mathcal{G}^{k}(p)$. 
Proposition 3 Let $k=1,2, . . n$. The following conditions are equivalent:

(i) $F \succcurlyeq_{R B D(2)[\mathbf{z}]} G$

(ii) $Q_{k}^{F} \cdot \mathcal{P} \mathcal{G}_{F}^{k}\left(p / Q_{k}^{F}\right) \leq Q_{k}^{G} \cdot \mathcal{P} \mathcal{G}_{G}^{k}\left(p / Q_{k}^{G}\right)$ for all $p \in\left[0, \min \left\{Q_{k}^{F}, Q_{k}^{G}\right\}\right]$

and $\sum_{i=1}^{k} q_{i}^{F} H\left(F_{i}, z_{i}\right) \cdot I\left(F_{i}, z_{i}\right) \leq \sum_{i=1}^{k} q_{i}^{G} H\left(G_{i}, z_{i}\right) \cdot I\left(G_{i}, z_{i}\right)$.

Note that when dominance is evaluated at the last stage, condition (ii) becomes $\mathcal{P G}_{F}(p) \leq \mathcal{P} \mathcal{G}_{G}(p)$ for all $p \in[0,1]$. That is, we recover the standard poverty dominance condition applied to homogeneous populations. This condition is not obtained for poverty comparisons over heterogeneous distributions, either in the standard approach pioneered by Atkinson (1992) or in the dual approach to sequential poverty dominance suggested in Zoli (2000). In the former case, at the final stage of comparisons, second degree poverty dominance coincides with generalized Lorenz dominance for all incomes below the lowest poverty line (that is the poverty line $z_{n}$ of the least needy group). This condition turns out to be equivalent to APGP curve dominance for the unique poverty line $z_{n}$. In the latter case the second degree poverty dominance is obtained through weighted averages of the APGP curves of each group evaluated w.r.t. the group poverty line.

Moreover, if the marginal distribution of needs is fixed, as in Atkinson and Bourguignon (1987) and Atkinson (1992), then the previous conditions require that at each stage $k$ the poverty gap curves of the neediest $k$ groups are compared. That is, if $q_{i}^{F}=q_{i}^{G}$ for all $i$, so that $Q_{k}^{F}=Q_{k}^{G}$ for all $k$, and noting that $\mathcal{P G}_{F}^{k}(1)=$ $\sum_{i=1}^{k} q_{i}^{F} H\left(F_{i}, z_{i}\right) \cdot I\left(F_{i}, z_{i}\right)$, condition (ii) in Proposition 3 becomes equivalent to $\mathcal{P} \mathcal{G}_{F}^{k}(p) \leq \mathcal{P} \mathcal{G}_{G}^{k}(p)$ for all $p \in[0,1]$.

Corollary 1 If $q_{i}^{F}=q_{i}^{G}$ for all $i$, then $R B D(j) \Longleftrightarrow S P G D(j)$ for $j=1,2$.

Similar results can be found for relative poverty comparisons, just changing the perspective from absolute to relative poverty gaps. In that case, the standard relative poverty gap profile curves (where poverty gaps are normalized by the poverty line) have to be applied in order to check dominance. If relative poverty comparisons are applied, the appropriate reference level at which to make comparisons is the normalized income $x / z_{i}$. In this case since the upper bound of the range of $x / z_{i}$ is 1 in all groups, the standard approach and the one suggested here both provide the same criteria. It should however be pointed out that in order to establish these criteria in the relative case, the characterization of the individual's deprivation functions must involve comparisons of "normalized transfers" (of amounts $\delta / z_{i}$ depending on the group poverty line $z_{i}$ ). As also pointed out in Atkinson (1992, p. 7) however, looking at absolute poverty gap (and income) levels provides the most natural perspective to approach the issue of poverty measurement in an heterogenous population framework.

Given the equivalence between $R B D$ and $S P G D$ even when marginal distributions of needs differ, we will interchangeably use their notations in the remaining part of the paper. 


\subsection{Variable poverty lines}

Our previous results depend on the setting of an appropriate group reference level (i.e. poverty line). Although our procedure is less demanding than identifying cardinal equivalence scales to implement between groups poverty comparisons, the setting of the poverty lines may turn out to be a source of disagreement between policy makers. When agreement is not reached on the appropriate set of poverty lines, a possible solution is to make comparisons considering vectors of ordered poverty lines, each one of them defined within a range.

In general this type of comparison turns out to be problematic for the approach we follow. This is hardly surprising. The logic of our approach is based on the definition of a reference point, the poverty line, within each group, therefore once uncertainty about the appropriate value of the reference point is introduced then also the between-groups comparisons are affected. In particular, since individuals are compared between groups for a given absolute poverty gap, if a poverty line changes then the income levels that are pairwise comparable between groups change, thereby affecting all the dominance conditions! However, if uncertainty about the poverty lines takes a particular specification then it is possible to provide a clearcut result. If the poverty lines all change by the same amount, the between-groups pairwise comparisons are still made at the same income levels, the only additional implication being that potentially more individuals become poor as poverty lines increase; therefore new portions of the income distributions have to be taken into account in the evaluation.

Suppose that every poverty line $z_{i}$ can be decomposed into two components, a fixed minimum level $\tilde{z}_{i}$ which is defined and is such that $\tilde{z}_{i} \geq \tilde{z}_{i+1}$ and a positive additive element $\beta$ which is common to the evaluation of all poverty lines, that is $z_{i}=\tilde{z}_{i}+\beta$. It follows that $z_{i}-z_{j}=\tilde{z}_{i}-\tilde{z}_{j}$, that is, there is no disagreement on the gap between the various poverty lines of different groups but only on their absolute values.

In order to analyze the implications that changes in $\beta$ could have on the final ranking, we first discuss the case of a homogeneous population. Consider the condition $\int_{\gamma}^{z_{i}}\left[\phi_{i}^{F}\left(t, z_{i}\right)-\phi_{i}^{G}\left(t, z_{i}\right)\right] d t \leq 0$ associated with $\operatorname{RBD}(1)$. In the case of a homogeneous population $q_{i}^{F}=1$, so that $\phi_{i}^{F}\left(\gamma, z_{i}\right)=F_{i}\left(z_{i}-\gamma\right)$. If the poverty line increases by $\beta>0$ then the previous condition could be shifted and we can check that

$$
\int_{\gamma}^{z_{i}+\beta}\left[\phi_{i}^{F}\left(t, z_{i}+\beta\right)-\phi_{i}^{G}\left(t, z_{i}+\beta\right)\right] d t=\int_{\gamma-\beta}^{z_{i}}\left[\phi_{i}^{F}\left(t, z_{i}\right)-\phi_{i}^{G}\left(t, z_{i}\right)\right] d t
$$

for all $\gamma \geq \beta$. It follows that checking for poverty dominance of $F_{i}$ over $G_{i}$ evaluated for the poverty line $z_{i}+\beta$ carries with it dominance also for the poverty line $z_{i}$. Once we move to an heterogeneous population comparison, if all poverty lines are shifted by the same amount the same line of reasoning can be extended. We then have that $\int_{\gamma}^{z_{i}+\beta} \Delta \phi_{i}\left(t, z_{i}+\beta\right)=\int_{\gamma-\beta}^{z_{i}} \Delta \phi_{i}\left(t, z_{i}\right) d t$ for all $i=1,2,3, . . n$ and for all $\gamma \geq \beta$, where 
$\Delta \phi_{i}\left(\gamma, z_{i}\right)=\phi_{i}^{F}\left(\gamma, z_{i}\right)-\phi_{i}^{G}\left(\gamma, z_{i}\right)$. It follows that $\sum_{i=1}^{k} \int_{\gamma}^{\gamma^{*}} \Delta \phi_{i}\left(t, z_{i}+\beta\right) d t \leq 0$ for all $k=1,2, . . n$ and all $\gamma \geq 0$ implies that $\sum_{i=1}^{k} \int_{\gamma}^{\gamma^{*}} \Delta \phi_{i}\left(t, z_{i}\right) d t \leq 0$ for all $k=1,2, . . n$ and all $\gamma \geq 0$. Thus it is sufficient to check for dominance for the extreme value of the poverty line associated with the highest value of $\beta$ in order to have unanimous dominance for all the intermediate values of $\beta$, as formalized in the following remark.

Remark $1 P(F) \leq P(G)$ for all $u_{i}$ satisfying properties $A, A 1$ and A2 and for all $z_{i}=\tilde{z}_{i}+\beta$ where $\beta \in[0, \bar{\beta}]$ and $\tilde{z}_{i} \geq \tilde{z}_{i+1}$ if and only if $\sum_{i=1}^{k} \int_{\gamma}^{\gamma^{*}} \Delta \phi_{i}\left(t, \tilde{z}_{i}+\bar{\beta}\right) d t \leq 0$ for all $k=1,2, . . n$, and for all $\gamma>0$.

If the same assumptions on the poverty lines hold, it is possible to obtain a corresponding result for properties A and A1, i.e. under the conditions in Proposition 1. In this case the dominance condition will require: $\sum_{i=1}^{k} \Delta \phi_{i}\left(\gamma, \tilde{z}_{i}+\bar{\beta}\right) \leq 0$ for all $k=1,2, . . n$ and for all $\gamma>0$.

These results do not hold if the distances between the poverty lines are not maintained at fixed values.

\subsubsection{Poverty lines changing within ranges}

We show some further implications deriving from changing poverty lines when a distribution partitioned into only two groups is considered and then extend the comparisons to the general $n$ groups case.

Suppose that $z_{i} \in\left[z_{i}^{-}, z_{i}^{+}\right]$and $z_{i} \geq z_{i+1} \geq 0$, implying that $z_{i}^{-} \geq z_{i+1}^{-}$and $z_{i}^{+} \geq z_{i+1}^{+}$for all $i=1,2, . . n-1$. Let $Z^{n}\left(\mathbf{z}^{-}, \mathbf{z}^{+}\right)$denote the set of all ordered poverty lines $z_{1} \geq z_{2} \geq . . \geq z_{n} \geq 0$ satisfying such conditions for $n$ groups. If poverty dominance is required to be consistent with all possible $\mathbf{z} \in Z^{n}\left(\mathbf{z}^{-}, \mathbf{z}^{+}\right)$, then making use of Remark 1 it is possible to restrict the set of comparisons required.

In particular, if we suppose that $n=2$, at the first stage of the sequential comparison, it is sufficient to check for poverty dominance of the needier group at the higher poverty line $z_{1}^{+}$; while at the second stage it is sufficient to fix $z_{1}=z_{1}^{+}$and check for dominance for all $z_{2} \in\left[z_{2}^{-}, z_{2}^{+}\right]$and also to fix $z_{2}=z_{2}^{+}$and check for poverty dominance when $z_{1} \in\left[z_{2}^{+}, z_{1}^{+}\right]$. All the other comparisons associated with the remaining vectors in $Z^{2}\left(\mathbf{z}^{-}, \mathbf{z}^{+}\right)$will be implied by these. The conditions for robust poverty comparisons valid for all $\mathbf{z} \in Z^{2}\left(\mathbf{z}^{-}, \mathbf{z}^{+}\right)$unfortunately become more complicated as the number of groups increases.

Proposition 4 If $n=2, P(F) \leq P(G)$ for all $u_{i}$ satisfying properties $A, A 1$ and $A 2$ and for all $\mathbf{z} \in Z^{2}\left(\mathbf{z}^{-}, \mathbf{z}^{+}\right)$if and only if:

(i) $\int_{\gamma}^{z_{1}^{+}} \Delta \phi_{1}\left(t, z_{1}^{+}\right) d t \leq 0$ for all $\gamma>0$, and and

(iia) $\int_{\gamma}^{z_{1}^{+}} \Delta \phi_{1}\left(t, z_{1}^{+}\right) d t+\int_{\gamma}^{z_{1}^{+}} \Delta \phi_{2}\left(t, z_{2}\right) d t \leq 0$ for all $\gamma>0$ and all $z_{2} \in\left[z_{2}^{-}, z_{2}^{+}\right]$,

(iib) $\int_{\gamma}^{z_{1}} \Delta \phi_{1}\left(t, z_{1}\right) d t+\int_{\gamma}^{z_{1}} \Delta \phi_{2}\left(t, z_{2}^{+}\right) d t \leq 0$ for all $\gamma>0$ and all $z_{1} \in\left[\min \left\{z_{1}^{-}, z_{2}^{+}\right\}, z_{1}^{+}\right]$. 
Even for the simplest $n=2$ case we thus have an infinity of dominance conditions to check associated with the values of $z_{2} \in\left[z_{2}^{-}, z_{2}^{+}\right]$in (iia), and $z_{1} \in$ $\left[\min \left\{z_{1}^{-}, z_{2}^{+}\right\}, z_{1}^{+}\right]$in (iib). It is however possible to derive an algorithm that will allow to check all these conditions in a finite number of steps both for 1st and 2nd degree sequential reference-based dominance (SPGD). We illustrate first the algorithm for the $n=2$ case, then we provide its formula for the generic case proved in the Appendix. The algorithm suggested in Bourguignon (1989) for welfare dominance can be obtained as a special case of ours.

Let $\Delta_{i}^{[1]}(x):=q_{i}^{F} F_{i}(x)-q_{i}^{G} G_{i}(x)$, and $\Delta_{i}^{[2]}(x):=\int_{0}^{x} \Delta_{i}^{[1]}(t) d t$ for groups $i=1,2$. For the first group we consider the following transformation of $\Delta_{1}^{[j]}(x)$ for $j=1,2$ :

$$
\hat{\Delta}_{1}^{[j]}\left(x ; \mathbf{z}^{-}, \mathbf{z}^{+}\right):=\max _{t \in\left[x+\max \left\{0 ; z_{1}^{-}-z_{2}^{+}\right\}, z_{1}^{+}+\min \left\{0 ; x-z_{2}^{-}\right\}\right], t \neq z_{1}^{+}}\left\{\Delta_{1}^{[j]}(t)\right\} .
$$

At the second stage of the reference-based dominance of order $j=1,2$, the comparisons for a given poverty gap $\gamma>0$ require adding $\Delta_{1}^{[j]}\left(z_{1}-\gamma\right)$ to $\Delta_{2}^{[j]}\left(z_{2}-\gamma\right)$. Letting $\gamma=z_{2}-x$ for $x \in\left[0, z_{2}^{+}\right)$, and rewriting the condition, we get $\Delta_{1}^{[j]}\left(x+z_{1}-z_{2}\right)+\Delta_{2}^{[j]}(x)$. Given that $z_{1} \geq z_{2}$, and $z_{2} \in\left[z_{2}^{-}, z_{2}^{+}\right]$, and $z_{1} \in\left[z_{2}^{+}, z_{1}^{+}\right]$the algorithm requires to compare for any admissible $x$ in the argument of $\Delta_{2}^{[j]}$ the set of values of $\Delta_{1}^{[j]}$ in the interval $\left[x+\max \left\{0 ; z_{1}^{-}-z_{2}^{+}\right\}, z_{1}^{+}+\min \left\{0 ; x-z_{2}^{-}\right\}\right]$excluding the case of $x=z_{1}^{+}$ given that this corresponds to $\gamma=0$. For a given value of $x \in\left[0, z_{2}^{+}\right)$, the dominance condition necessarily has to be satisfied for the maximum of $\Delta_{1}^{[j]}$ in the interval, and therefore is also satisfied for all the values in the interval.

Proposition 5 Let $n=2$, and $j=1,2 . F \succcurlyeq_{S P G D(j)[\mathbf{z}]} G$ for all $\mathbf{z} \in Z^{2}\left(\mathbf{z}^{-}, \mathbf{z}^{+}\right)$iff:

(i) $\Delta_{1}^{[j]}(x) \leq 0$ for all $x \in\left[0, z_{1}^{+}\right)$;

(ii) $\Delta_{2}^{[j]}(x)+\hat{\Delta}_{1}^{[j]}\left(x ; \mathbf{z}^{-}, \mathbf{z}^{+}\right) \leq 0$ for all $x \in\left[0, z_{2}^{+}\right)$.

Note that if the ranges of poverty lines overlap, i.e. $z_{1}^{-} \leq z_{2}^{+}$, then the value of $z_{1}^{-}$does not play any role in the dominance condition, while $z_{2}^{-}$appears in the definition of the interval for $t$ in (6). The dominance conditions derived are valid for any value $z_{1}^{-}$of the lower bound of poverty lines of group 1 as long as $z_{1}^{-} \geq z_{2}^{-}$. A special case is when $z_{1}^{-}=z_{2}^{-}=0$, for these comparisons the interval in (6) is modified to $\left[x, z_{1}^{+}\right)$. If $\Delta_{1}^{[1]}(x) \leq 0$ for all $x \in\left[0, z_{1}^{+}\right)$but the proportion of individuals strictly below the poverty line is the same within group 1 in both populations then $\max _{t \in\left[x, z_{1}^{+}\right)}\left\{\Delta_{1}^{[1]}(t)\right\}=0$ for all $x \in\left[0, z_{2}^{+}\right)$. It follows that at the second stage of comparison $\Delta_{2}^{[1]}(x) \leq 0$ is required to hold for all $x \in\left[0, z_{2}^{+}\right)$. Similarly if $j=2$ and the average poverty gap is the same within group 1 in both populations, i.e. $\max _{t \in\left[x, z_{1}^{+}\right)}\left\{\Delta_{1}^{[2]}(t)\right\}=0$, the dominance condition at the second stage boils down to $\Delta_{2}^{[2]}(x) \leq 0$ for all $x \in\left[0, z_{2}^{+}\right)$. In both cases the sequential dominance conditions cannot improve the power of the restrictive conditions requiring dominance in each group. 
We illustrate here the algorithm for the generic case with $n$ groups under the assumption that $z_{i}^{-}=0$ for all $i$. This assumption is sufficient to get an easily implementable algorithm for checking dominance in the $n$ groups. A more general algorithm associated with the case where $z_{i}^{-}=z^{-} \geq 0$ for all $i$ is also proved in the Appendix.

Under the assumption $z_{i}^{-}=0$ for all $i$ the algorithm is obtained making use of functions $\hat{\Delta}_{i}^{[j]}$ derived in (6) for $n=2$. For $i=0,1,2, . . n-1$ let

$$
\tilde{\Delta}_{i+1}^{[j]}\left(x ; \mathbf{z}^{-}, \mathbf{z}^{+}\right):=\Delta_{i+1}^{[j]}(x)+\hat{\Delta}_{i}^{[j]}\left(x ; \mathbf{z}^{-}, \mathbf{z}^{+}\right) \text {for all } x \in\left[0, z_{i+1}^{+}\right],
$$

where $\hat{\Delta}_{0}^{[j]}\left(x ; \mathbf{z}^{-}, \mathbf{z}^{+}\right):=0$ for all $x$, while for $i=1,2, . . n$ we define

$$
\hat{\Delta}_{i}^{[j]}\left(x ; \mathbf{z}^{-}, \mathbf{z}^{+}\right):=\max _{t \in\left[x ; z_{i}^{+}\right)}\left\{\tilde{\Delta}_{i}^{[j]}\left(t ; \mathbf{z}^{-}, \mathbf{z}^{+}\right)\right\} .
$$

Proposition 6 Let $j=1,2$, and $z_{i}^{-}=0$ for all $i$. The following statements are equivalent:

(i) $F \succcurlyeq_{S P G D(j)[\mathbf{z}]} G$ for all $\mathbf{z} \in Z^{n}\left(\mathbf{0}, \mathbf{z}^{+}\right)$,

(ii) $\hat{\Delta}_{i}^{[j]}\left(0 ; \mathbf{0}, \mathbf{z}^{+}\right) \leq 0$ for all $i=1,2, \ldots n$.

Note that the condition $\hat{\Delta}_{i}^{[j]}\left(0 ; \mathbf{z}^{-}, \mathbf{z}^{+}\right) \leq 0$ for $i=1,2$ are equivalent to the two conditions in Proposition 5 once $z_{i}^{-}=0$ for all $i$. In the general case where the $z_{i}^{-}$'s are positive and may differ the conditions are less restrictive given that $\hat{\Delta}_{1}^{[j]}\left(x ; \mathbf{z}^{-}, \mathbf{z}^{+}\right)$ does not consider the maximum for values of $t \in\left[x, x+z_{1}^{-}-z_{2}^{+}\right)$if $z_{1}^{-}>z_{2}^{+}$and/or for $t \in\left(z_{1}^{+}+x-z_{2}^{-}, z_{1}^{+}\right)$if $x<z_{2}^{-}$. Both sets of values for $\Delta_{1}^{[j]}(x)$ are not considered if $x<z_{2}^{-} \leq z_{2}^{+}<z_{1}^{-} \leq z_{1}^{+}$, while if $z_{2}^{-} \leq x \leq z_{2}^{+}<z_{1}^{-} \leq z_{1}^{+}$only the first set of values is ruled out from the procedure at the second stage. If $x<z_{2}^{-} \leq z_{1}^{-} \leq z_{2}^{+} \leq z_{1}^{+}$it is the second set of values of $\Delta_{1}^{[j]}(x)$ that is ruled out. And if $z_{2}^{-} \leq z_{1}^{-} \leq z_{2}^{+} \leq z_{1}^{+}$and $z_{2}^{-} \leq x$ the conditions for dominance at the second stage (for $n=2$ ) coincide with those derived for $z_{i}^{-}=0$ for all $i$.

An interesting corollary can be derived from the previous proposition. Suppose that $\left(\mathbf{z}^{-}, \mathbf{z}^{+}\right)$is such that $z_{i} \geq z_{i+1}, z_{i}^{+}=\bar{z}>0$ for all $i$ (i.e. $\mathbf{z}^{+}=\bar{z} \mathbf{1}$ where $\mathbf{1}$ denotes the $n$-dimensional vector of ones), and $z_{i}^{-}=0$ for all $i$, i.e. $\mathbf{z}^{-}=\mathbf{0}$. It follows that

$$
\hat{\Delta}_{i}^{[j]}(x ; \mathbf{0}, \bar{z} \mathbf{1}):=\max _{t \in[x, \bar{z})}\left\{\tilde{\Delta}_{i}^{[j]}(t)\right\} .
$$

Corollary 2 Let $j=1,2$, and $z_{i}^{+}=\bar{z}>z_{i}^{-}=0$ for all $i$. The following statements are equivalent:

(i) $F \succcurlyeq_{S P G D(j)[\mathbf{z}]} G$ for all $\mathbf{z} \in Z^{n}(\mathbf{0}, \bar{z} \mathbf{1})$,

(ii) $\hat{\Delta}_{i}^{[j]}(0 ; \mathbf{0}, \bar{z} \mathbf{1}) \leq 0$ for all $i=1,2, \ldots n$, where $\tilde{\Delta}_{i}^{[j]}(x)$ is obtained as in (9) making use of (7).

This algorithm corresponds to $S P G D$ of order $j=1,2$ for all ranked poverty lines in $[0, \bar{z}]$. If $j=2$ and $q_{i}=q_{i}^{F}=q_{i}^{G}$ for all $i$ it coincides with the algorithm suggested in Bourguignon (1989) for welfare dominance. 


\section{Relationship to existing welfare literature}

Bourguignon (1989) suggested a general stochastic dominance criterion valid for multivariate welfare comparisons where the population is partitioned into groups ranked according to family size (needs), as in this work. The criterion suggested is applied to comparisons between distributions (bounded at $\bar{x}$ ) with identical marginal distributions of needs, i.e. such that $q_{i}=q_{i}^{F}=q_{i}^{G}$ for all $i$. We first show that our criterion can be considered as a special case of the one suggested by Bourguignon. Most importantly we also show that when poverty lines are allowed to change without any boundaries then $\operatorname{SPGD}(2)$ and Bourguignon Dominance (BD) are equivalent.

We will write $F \succcurlyeq_{B D} G$ to denote that distribution $F$ dominates $G$ according to the Bourguignon Dominance (BD) condition.

Definition 5 (Bourguignon-Dominance) For all $F, G \in \mathcal{F}$ s.t. $q_{i}^{F}=q_{i}^{G}=q_{i}$

$$
F \succcurlyeq_{B D} G \Longleftrightarrow \sum_{i=1}^{n} q_{i} \int_{0}^{x_{i}}\left[F_{i}(t)-G_{i}(t)\right] d t \leq 0
$$

for all $x_{1}, x_{2}, . . x_{n}$ s.t. $\bar{x} \geq x_{1} \geq x_{2} \geq \ldots \geq x_{n} \geq 0$.

In our context, if $q_{i}=q_{i}^{F}=q_{i}^{G}$ for all $i$ then the dominance conditions obtained in Propositions 1 (part ii) and shown to be equivalent to $\operatorname{SPGD}(2)$ in Proposition 3 are also equivalent to a restricted form of Bourguignon-dominance which obtains when considering only those values of $x_{1}, x_{2}, . ., x_{i}, . ., x_{n-1}, x_{n}$ such that, for a given vector of ordered poverty lines $\mathbf{z}$ :

$$
z_{i}-x_{i}=c \geq 0, \quad x_{j}=0 \text { for all } c \geq 0, \text { all } i \leq k, \text { all } j>k, \text { and for all } k=1,2, . . n \text {. }
$$

The standard sequential poverty dominance $[\operatorname{SPD}(2)]$ criteria we have enumerated can also be interpreted as special cases of Bourguignon (1989). For instance the version of the $\operatorname{SPD}(2)$ criterion where $q_{i}=q_{i}^{F}=q_{i}^{G}$ can be obtained, for a given vector of poverty lines $\mathbf{z}$ where $z_{i} \geq z_{i+1}$, by restricting Bourguignon's conditions to hold for vectors of $x_{i}$ such that:

$$
0 \leq x_{i}=x_{j} \leq z_{k}, \quad x_{t}=0 \text { for all } i, j \leq k, \text { all } t>k \text { and for all } k=1,2, . . n .
$$

This result holds even if we consider dominance for all poverty lines below those considered and such that the ranking of the lines is preserved.

When $S P G D(2)$ comparisons are extended in order to take into account any ranked vector of positive poverty lines the connection with BD dominance becomes clear.

Proposition 7 If $q_{i}=q_{i}^{F}=q_{i}^{G}, F \succcurlyeq_{S P G D(2)[\mathbf{z}]} G$ for all $z_{1}, z_{2}, . ., z_{n}$ s.t. $\bar{z} \geq z_{1} \geq$ $z_{2} \geq \ldots \geq z_{n}>0$ if and only if $F \succcurlyeq_{B D} G$. 
Therefore, the numerical algorithm suggested by Bourguignon to test BD will test also $S P G D(2)[\mathbf{z}]$ (when $q_{i}=q_{i}^{F}=q_{i}^{G}$ ) for all ranked poverty line vectors, as also shown in Corollary 2.

It is worth pointing out that this is not the case if the $S P D(2)$ conditions are checked for all $z_{1} \geq z_{2} \geq \ldots \geq z_{n}>0$. It can be shown that $F \succcurlyeq_{B D} G \Longrightarrow F \succcurlyeq_{S P D(2)[\mathbf{z}]}$ $G$ for all $z_{1} \geq z_{2} \geq \ldots \geq z_{n}>0$ but the implication cannot go in the opposite direction, since as a corollary of the previous proposition $F \succcurlyeq_{S P G D(2)[\mathbf{z}]} G \Longrightarrow F \succcurlyeq_{S P D(2)[\mathbf{z}]} G$ when dominance is evaluated for all $z_{1} \geq z_{2} \geq \ldots \geq z_{n}>0$.

Fleurbaey et al. (2003) generalize the analysis in Bourguignon (1989) assuming bounded (relative) equivalence scale relativities $1 \leq \alpha_{i} \leq \beta_{i}$. According to their assumptions the social marginal value of income in group $i$ for an income unit with income $x$ is higher [lower] than that of an income unit in group $i+1$ with income below $x / \alpha_{i}$ [above $x / \beta_{i}$ ]. As pointed out in the paper their dominance criterion requires to compare the average poverty gaps for the whole population, $I(F, \mathbf{z})=\mathcal{P} \mathcal{G}_{F}^{n}(1)=$ $\sum_{i=1}^{n} q_{i}^{F} H\left(F_{i}, z_{i}\right) \cdot I\left(F_{i}, z_{i}\right)$. More precisely $F$ dominates $G$ iff $I(F, \mathbf{z}) \leq I(G, \mathbf{z})$ for all ranked vectors of poverty lines such that $\alpha_{i} z_{i-1} \leq z_{i} \leq \beta_{i} z_{i-1}$. Exploiting the analogy with Fleurbaey et al. (2003), the idea of comparing the social marginal value of income at different income levels of poor individuals exhibiting different needs by looking at the poverty gaps is intimately connected with setting absolute equivalence scales. ${ }^{8}$ We can identify a sequence of values $\tilde{\theta}_{i}$ such that once the poverty line $z_{n}$ of the least needy group is fixed we get $z_{i}=z_{n}+\tilde{\theta}_{i}$. Recalling that we have derived $\theta_{i}:=z_{1}-z_{i}$, we get $\tilde{\theta}_{i}:=\theta_{i}-\theta_{n}$. The sequence of values $\tilde{\theta}_{i}$ identifies the absolute equivalence scales. Property A1 imposes that the social marginal value of income in group $i$ for a poor income unit with income $x$ is higher than that of a poor income unit in group $i+1$ with income below $x+\theta_{i}-\theta_{i+1}$. These conditions are also consistent with considering comparisons of distributions of equivalent incomes obtained making use of absolute equivalence scales that are decreasing in nominal income for incomes below the poverty line. ${ }^{9}$

We can also make an analogy with Bazen and Moyes' (2003) modification of the Atkinson and Bourguignon (1987) and Jenkins and Lambert (1993) results. This requires in our setting to modify (3) and Property A s.t. $u_{i}(0)=a_{i} \geq 0$ in order to quantify the "stigma effect" of being poor, and furthermore to assume that $a_{i} \geq a_{i+1}$ for all $i=1,2, . . n-1$. The dominance condition in Proposition 1 will consequently have to include $\sum_{i=1}^{k} q_{i}^{F} H\left(F_{i}, z_{i}\right) \leq \sum_{i=1}^{k} q_{i}^{G} H\left(G_{i}, z_{i}\right)$ for all $k=1,2, . . n$. This additional requirement has been derived also in Duclos, et al. (2003) and used to supplement the SPD conditions in order to account for dominance criteria that

\footnotetext{
${ }^{8}$ For a recent analysis of the normative implication of using equivalence scales in welfare (and inequality) comparisons of heterogeneous populations see Ebert and Moyes (2003).

${ }^{9}$ We thank Krishna Pendakur for this remark. This class of equivalence scales is consistent with the finding in Donaldson and Pendakur (2004) that relative equivalence scales are decreasing in expenditure levels. However, it is an open question wether for low expenditure levels the decrease in the value of the relative equivalence scales is so sharp as to imply that equivalence scales can be decreasing also in absolute terms.
} 
are consistent with discontinuous poverty indices like the headcount ratio or those introduced in Bourguignon and Fields (1997). Note that this condition is enough, when required to hold for all ordered poverty lines in $[0, \bar{z}]$, to induce the analogue of $\mathrm{BD}$ for comparisons based on first order dominance as obtained in Proposition 6 letting $j=1$, which is equivalent to $\operatorname{SPGD(1)~(and~therefore~will~imply~} \operatorname{SPGD}(2)$ ) checked for all poverty lines considered.

The results presented for SPGD can also be extended to consider higher orders of dominance as done for welfare comparisons in Lambert and Ramos (2002).

\subsection{Intermediate Dominance}

Some intermediate dominance conditions, stronger than SPGD(1) and SPGD(2) respectively, can also be devised. These will be related to conditions obtained analyzing the problem comparing individuals at income levels as in Atkinson (1992) rather than at poverty gap levels as in this paper. The approach is similar in spirit to the one adopted by Bourguignon (1989) to weaken the sequential dominance conditions in Atkinson and Bourguignon (1987).

In order to get the first result we need to introduce the following condition:

Property A0 $u_{i}(\gamma) \geq u_{i+1}(\gamma) \geq 0$ for all $\gamma>0$, and all $i=1,2, . . n-1$.

Note that this condition is implied by A and A1, but once the Weak Monotonicity axiom WM is substituted for A1 then A, WM and A0 are independent. It is precisely the combination of these conditions that we will consider to obtain a stronger dominance condition than $S P G D(1)$. In order to strengthen $\operatorname{SPGD}(2)$ we will instead consider the combination of conditions A, A1 and WPT. The latter property is clearly weaker than the property A2 used in conjunction with A and A1 to characterize $\operatorname{SPGD}(2)$.

To present the results we make use of the notation adopted in Proposition 6 and consider an ordered poverty vector $\mathbf{z} \in Z^{n}$. Recall that $\Delta_{i}^{[1]}\left(x_{i}\right):=q_{i}^{F} F_{i}\left(x_{i}\right)-$ $q_{i}^{G} G_{i}\left(x_{i}\right)$, and $\Delta_{i}^{[2]}\left(x_{i}\right):=\int_{0}^{x_{i}} \Delta_{i}^{[1]}(t) d t$. We consider $j=1,2$ and for $i=0,1,2, . . n-1$ we let

$$
\tilde{\Delta}_{i+1}^{[j]}(x ; \mathbf{z}):=\Delta_{i+1}^{[j]}(x)+\hat{\Delta}_{i}^{[j]}\left(x+\delta_{i} ; \mathbf{z}\right) \text { for all } x \in\left[0, z_{i+1}\right),
$$

where $\hat{\Delta}_{0}^{[j]}(x ; \mathbf{z}):=0$ for all $x, z_{0}:=z_{1}$ and $\delta_{i}:=z_{i}-z_{i+1}$ while for $i=1,2, . . n$ we define

$$
\hat{\Delta}_{i}^{[j]}(x ; \mathbf{z}):=\max _{t \in\left[x ; z_{i}\right)}\left\{\tilde{\Delta}_{i}^{[j]}(t ; \mathbf{z})\right\} .
$$

Note that the condition $\hat{\Delta}_{i}^{[j]}(0 ; \mathbf{z}) \leq 0$ for all $i$ is equivalent to impose that $\tilde{\Delta}_{i}^{[j]}\left(x_{i} ; \mathbf{z}\right) \leq 0$ for all $i$ and for all $x_{i} \in\left[0 ; z_{i}\right)$. These conditions require that between group stochastic dominance comparisons are made pairing an income level in a less needy group with all income levels in needier groups that are associated with the same or a lower poverty gap level. 
Proposition 8 For a fixed $\mathbf{z} \in Z^{n}, P(F) \leq P(G)$ for all $u_{i}$ satisfying properties $A$, $A 0$, and $W M$ if and only if $\hat{\Delta}_{i}^{[1]}(0 ; \mathbf{z}) \leq 0$ for all $i=1,2, \ldots n$.

Proposition 9 For a fixed $\mathbf{z} \in Z^{n}, P(F) \leq P(G)$ for all $u_{i}$ satisfying properties $A$, $A 1$ and WPT if and only if $\hat{\Delta}_{i}^{[2]}(0 ; \mathbf{z}) \leq 0$ for all $i=1,2, \ldots n$.

As is clear from Proposition 6 the above dominance conditions are similar to $F \succcurlyeq_{S P G D(j)[\mathbf{z}]} G$ for all poverty lines in $Z^{n}(\mathbf{0}, \mathbf{z})$, for $j=1$ and $j=2$ respectively. But we have a main difference, the results in Proposition 6 require to make stochastic dominance comparisons between an income level in a less needy group and all higher income levels in needier groups, while the results in Propositions 8 and 9 require that these comparisons are made taking into account all income levels in needier groups that are associated with non higher poverty gap levels. When poverty lines do not coincide between groups then the results in Proposition 6 imply those in Propositions 8 and 9 but the reverse may not be true.

Corollary 3 Consider $u_{i}$ satisfying property $A$,

(i) $P(F) \leq P(G)$ for all $u_{i}$ satisfying $A 1$ and for all $\mathbf{z} \in Z^{n}\left(\mathbf{0}, \mathbf{z}^{+}\right) \Longrightarrow P(F) \leq$ $P(G)$ for all $u_{i}$ satisfying $A 0$ and $W M$ for a fixed set of poverty lines $\mathbf{z}^{+}$.

(ii) $P(F) \leq P(G)$ for all $u_{i}$ satisfying $A 1$ and $A 2$ for all $\mathbf{z} \in Z^{n}\left(\mathbf{0}, \mathbf{z}^{+}\right) \Longrightarrow$ $P(F) \leq P(G)$ for all $u_{i}$ satisfying $A 1$ and WPT for a fixed set of poverty lines $\mathbf{z}^{+}$.

If $\mathbf{z}^{+}=\bar{z} \mathbf{1}$ then the implications in both statements become equivalences.

The results obtained in Propositions 8 and 9 are in spirit similar to the one in Bourguignon (1989), in that the dominance conditions have to be checked for a range of values for the poverty lines, and if we require them to hold for $\mathbf{z}=\bar{z} \mathbf{1}$ then we get an analogue of the result obtained in Corollary 2.

Note that A, WM and WPT can also be equivalently expressed in terms of the poverty deprivation functions $p(x, z)$. Indeed when poverty lines are higher for needier groups then as shown in Claim 3 and Claim 4 in the Appendix properties A, A0, and WM imply $p_{i}\left(x, z_{i}\right) \geq p_{i+1}\left(x, z_{i+1}\right) \geq 0$, while A, A1 and WPT imply $p_{i}^{\prime}\left(x, z_{i}\right) \leq$ $p_{i+1}^{\prime}\left(x, z_{i+1}\right) \leq 0$ for all $i=1,2, . . n-1$ and for all $x$.

Therefore if we move the perspective of the analysis back to comparisons made at income levels, and denote with $\mathrm{A} 0^{*}$ and $\mathrm{A} 1 *$ the version of the above axioms specified in the income based framework then the class of poverty deprivation functions $p_{i}\left(x, z_{i}\right)$ consistent with $\mathrm{A}, \mathrm{WM}$ and $\mathrm{A} 0^{*}$ and with $\mathrm{A}, \mathrm{WPT}$ and $\mathrm{A}{ }^{*}$ are respectively larger than those obtained in the poverty gap framework.

The results in Claims 3 and 4 in the Appendix clarify the crucial distinction between the poverty gap and the income perspectives. According to claim 3 the dominance conditions derived imposing $\mathrm{A}$, WM and A0 applying the poverty gap perspective are implied by those obtained imposing $\mathrm{A}$, WM and $\mathrm{A} 0^{*}$ applying the income perspective. The poverty gap perspective leads to a potentially more decisive set of comparisons in that for a given income level in a lees needy group it rules 
out between groups comparability with levels of income in needier groups that are not lower but associated with higher poverty gap levels. If we strengthen WM with A1, then A0 is implied, but most importantly the two approaches give independent rankings. If we add WPT, Claim 4 shows again that the poverty gap dominance is implied by the one expressed at income levels. Finally if we strengthen the WPT requiring property A2 then the two approaches will lead to independent rankings.

In order to show these results systematically we list the properties derived within the poverty gap approach: A, A0, WM, BGP, WPT and BGTP and in addition to A, WM, and WPT we will consider also corresponding properties A0*, BGP*, and BGTP* defined using the income approach as in Atkinson (1992).

The following implications hold, if we take for granted A :

Remark 2 If $A$ holds then:

(i) $W M+B G P \Longrightarrow A 0$,

(ii) $W M+B G P^{*} \Longrightarrow A 0^{*}$.

We consider all poverty indices satisfying A and compare the two approaches (income vs. poverty gap), for a fixed set of poverty lines $\mathbf{z} \in Z^{n}$, we get the following results :

For fixed $\mathbf{z} \in Z^{n}$

\begin{tabular}{|c|c|c|}
\hline Poverty-Gap & & Income \\
\hline $\begin{array}{c}A 0+W M \\
\Downarrow\end{array}$ & $\Longleftarrow$ & $\begin{array}{c}A 0^{*}+W M \\
\Downarrow\end{array}$ \\
\hline$W M+B G P$ & $\stackrel{\rightleftarrows}{\not}$ & $W M+B G P^{*}$ \\
\hline$\Downarrow$ & & $\Downarrow$ \\
\hline $\begin{array}{c}W M+B G P \\
+W P T \\
\Downarrow\end{array}$ & $\Longleftarrow$ & $\begin{array}{c}W M+B G P^{*} \\
+W P T \\
\Downarrow\end{array}$ \\
\hline $\begin{array}{c}W M+B G P \\
+W P T+B G T P\end{array}$ & $\begin{array}{l}\rightleftarrows \\
\ldots\end{array}$ & $\begin{array}{c}W M+B G P^{*} \\
+W P T+B G T P^{*}\end{array}$ \\
\hline
\end{tabular}

Map A : "income approach" and "poverty gap approach" compared for dominance conditions derived for fixed poverty lines.

Note that once the impact of transfers on poverty deprivation is supposed to decrease as income increases (in accordance with WPT) then requiring a larger poverty reduction for a transfer taking place at the income level $x_{1}$ in group 1 with respect to a transfer taking place at $x_{2}<x_{1}$ in group 2 where both incomes are associated with the same poverty gap level, does imply that the same differential condition applies also if transfers occur at any income below $x_{1}$, therefore also for a level of income in 
group 1 identical to the level $x_{2}$ in group 2. For this reason, as also highlighted in Claim 4 in the Appendix, our perspective can be considered to give higher relevance to needs than the standard one following Atkinson's (1992) work. It should however be noticed that the implication does not hold if WPT is not assumed.

The comparisons in Map A can be extended weakening the previous dominance conditions requiring them to hold when evaluated over sets of alternative ranked poverty lines. In order to make the results comparable to the one of Bourguignon (1989) suppose to consider the set of ranked poverty lines $Z^{n}(\mathbf{0}, \bar{z} \mathbf{1})$. Then making use of Corollary 3 and combining it with Corollary 2 it will be possible to show that $\hat{\Delta}_{i}^{[j]}(0 ; \mathbf{z}) \leq 0$ for all $i$ and for all $\mathbf{z} \in Z^{n}(\mathbf{0}, \bar{z} \mathbf{1})$ if and only if $F \succcurlyeq_{S P G D(j)[\mathbf{z}]} G$ for all $\mathbf{z} \in Z^{n}(\mathbf{0}, \bar{z} \mathbf{1})$. More generally the following proposition holds:

Proposition 10 Let $j=1,2 . \hat{\Delta}_{i}^{[j]}(0 ; \mathbf{z}) \leq 0$ for all $i=1,2, \ldots$ and for all $\mathbf{z} \in$ $Z^{n}\left(\mathbf{0}, \mathbf{z}^{+}\right)$if and only if $F \succcurlyeq_{S P G D(j)[\mathbf{z}]} G$ for all $\mathbf{z} \in Z^{n}\left(\mathbf{0}, \mathbf{z}^{+}\right)$.

If we consider the set of $\mathbf{z} \in Z^{n}\left(\mathbf{0}, \mathbf{z}^{+}\right)$and we move the perspective to comparisons made at income levels then exploiting the analogies with the framework adopted in Bourguignon (1989) it will be possible to show that A, A0* and WM lead to the analogue of Bourguignon dominance for first order stochastic dominance comparisons i.e. we get $S P G D(1)[\mathbf{z}]$ for all $\mathbf{z} \in Z^{n}\left(\mathbf{0}, \mathbf{z}^{+}\right)$, while A, WM, BGP* and WPT lead to the dominance induced by $S P G D(2)[\mathbf{z}]$ for all $\mathbf{z} \in Z^{n}\left(\mathbf{0}, \mathbf{z}^{+}\right)$. Moreover, realizing that $\mathrm{A}, \mathrm{WM}$ and $\mathrm{BGP}^{*}$ lead to $S P D(1)[\mathbf{z}]$, applying dominance for all $\mathbf{z} \in Z^{n}\left(\mathbf{0}, \mathbf{z}^{+}\right)$ amounts, as shown by Atkinson (1992), to check $S P D(1)\left[\mathbf{z}^{+}\right]$. Similarly for A, WM, BGP*, WPT and BGTP* as shown in Jenkins and Lambert (1993) and Chambaz and Maurin (1998), the dominance requires that $S P D(2)\left[\mathbf{z}^{+}\right]$is satisfied. These conditions are clearly implied by $S P G D(j)[\mathbf{z}]$ for all $\mathbf{z} \in Z^{n}\left(\mathbf{0}, \mathbf{z}^{+}\right)$respectively for $j=1,2$.

To summarize we have that:

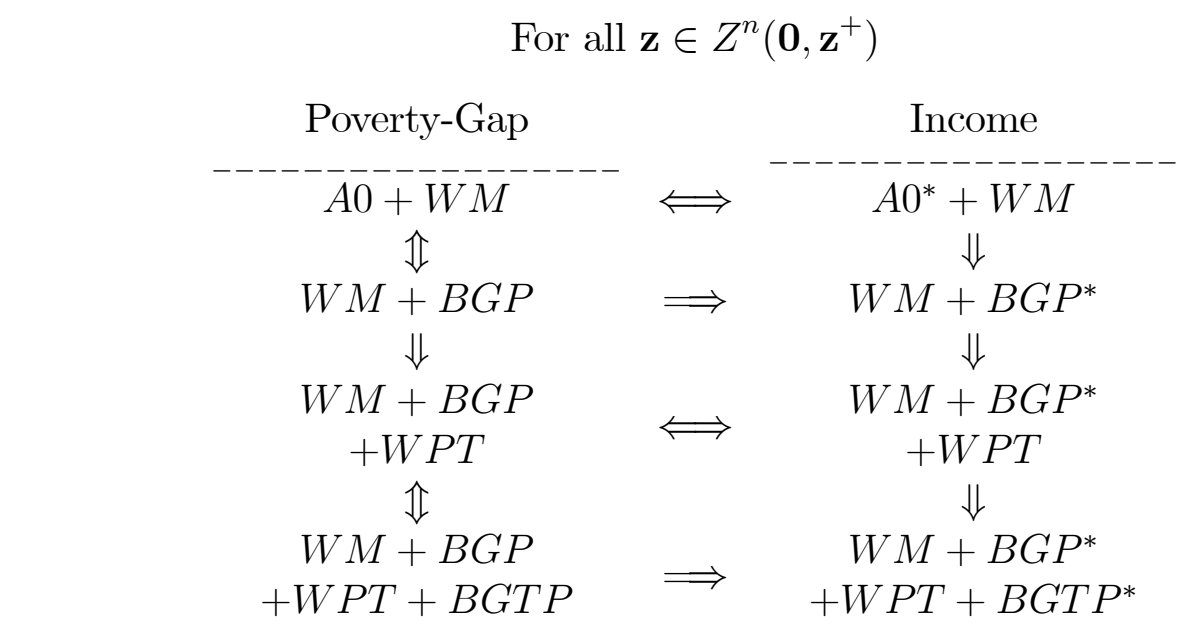

Map B : "income approach" and "poverty gap approach" compared for dominance conditions robust to changes in poverty lines 


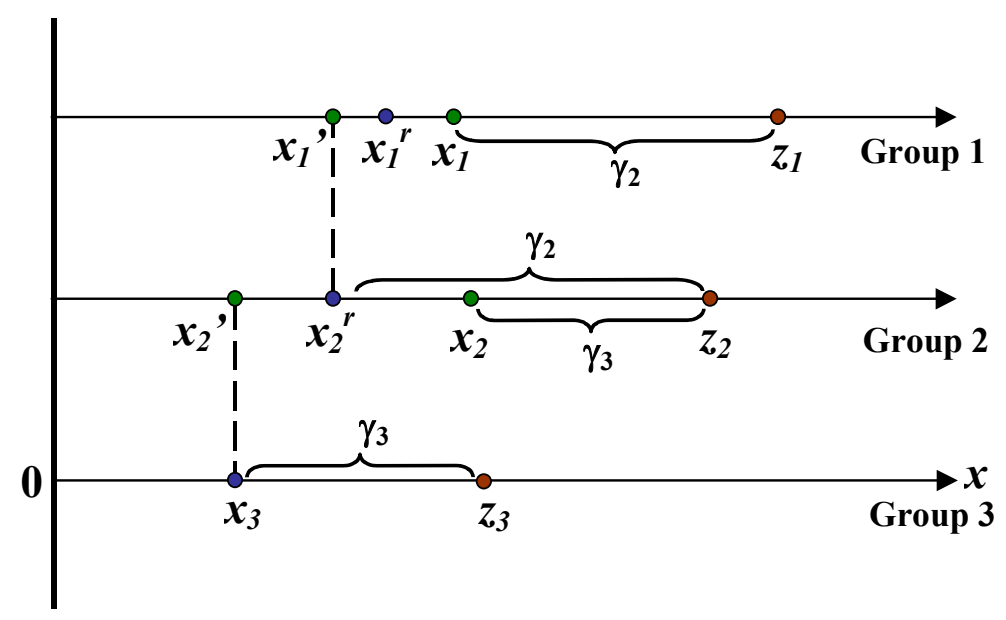

Figure 2: Comparability Sets for 3 Groups

For the special case of poverty lines defined in $Z^{n}\left(\mathbf{0}, \mathbf{z}^{+}\right)$the poverty gap perspective thus provides results that generalize the Bourguignon dominance condition and turn out to be more robust than those obtained in the framework relying on poverty comparability at income levels.

\section{Expanding the framework}

As discussed in the introduction, the approaches focusing on between groups poverty comparisons implemented at fixed income levels or at fixed poverty gap levels are extreme cases of more general type of poverty comparisons. For those comparisons, given a starting income in a less needy group, a comparable income is identified for the needier group adjacent in the ranking. This income is supposed not to be lower in absolute level than the initial one and should be associated with a poverty gap that is at least as large as the one in the initial group. This procedure can be expanded to the multiple groups case taking as starting income the one obtained for the new group and considering the associated income level in the next needier group, etc.. Since incomes are supposed not to be negative, and poverty lines are decreasing according to the group index, then it might well happen that for a needier group there exist low incomes that are not comparable with those in the less needy groups. Figure 2 illustrates the derivation of a set of comparable incomes in the case $n=3$ starting from a given $x_{3}$. The incomes $x_{3}<x_{2}^{r}<x_{1}^{r}$ identified in the figure are all associated with the same relative poverty gap.

Given the structure of the comparison, starting from $x_{3}$ the comparable incomes in group 2 should lie between $x_{2}^{\prime}\left(=x_{3}\right)$ and $x_{2}$ which is associated with the same 
absolute poverty gap $\left(\gamma_{3}\right)$ as $x_{3}$. Once $x_{2}^{r}$ has been selected then the comparable income in group 1 should lie between $x_{1}^{\prime}$ and $x_{1}$ where $x_{1}$ is associated with the same absolute poverty gap $\left(\gamma_{2}\right)$ as $x_{2}^{r}$.

For a given set of poverty lines we obtain in general a set $C$ of profiles of comparable incomes $x_{1} \geq x_{2} \geq \ldots \geq x_{k} \geq . . \geq x_{n *} \geq 0$ in groups $i=1,2, . . n^{*}$ for $n^{*} \leq n$ where each $x_{i}$ is included in $\left[0, z_{i}\right)$ such that there exists a unique profile of comparable incomes containing each $x_{i} \in\left[0, z_{i}\right)$. Moreover, we suppose that if for groups $i$ and $j$ the pair of values $\left(x_{i}, x_{j}\right)$ are comparable and similarly the values $\left(x_{i}^{\prime}, x_{j}^{\prime}\right)$ are comparable where $x_{i}>x_{i}^{\prime}$, then $x_{j}>x_{j}^{\prime}$.

Clearly different value judgements can lead to different sets $C$. For instance the Atkinson (1992) approach considers the set $C$ of all $\mathbf{x}$ such that $x_{1}=x_{2}=. .=x_{k}=$ $. .=x_{n *}=x$ where $x \in\left[0, z_{n^{*}}\right]$, while for our approach the set $C$ includes all $\mathbf{x}$ such that $x_{1} \geq x_{2} \geq \ldots \geq x_{k} \geq . . \geq x_{n *} \geq 0$ where $z_{1}-x_{1}=z_{2}-x_{2}=. .=z_{n^{*}}-x_{n *}$.

For the general approach it is possible to identify dominance conditions that are analogous to those previously derived for fixed poverty lines. Recall that $\Delta_{i}^{[1]}\left(x_{i}\right):=$ $q_{i}^{F} F_{i}\left(x_{i}\right)-q_{i}^{G} G_{i}\left(x_{i}\right)$, and $\Delta_{i}^{[2]}\left(x_{i}\right):=\int_{0}^{x_{i}} \Delta_{i}^{[1]}(t) d t$.

Definition 6 ((C)SD: (C) Sequential Poverty Dominance) For $\mathrm{z} \in Z^{n}$, and $F, G \in \mathcal{F}$ :

$F \succcurlyeq_{(C) S D(j)[\mathbf{z}]} G \Longleftrightarrow \sum_{i=1}^{k} \Delta^{[j]}\left(x_{i}\right) \leq 0$ for all $\mathbf{x} \in C$, all $k=1,2, \ldots n$.

A special case is obtained when relative poverty gaps are considered in order to identify comparable incomes. In this case $C$ includes all $\mathbf{x}$ s.t. $x_{i} / z_{i}=x_{n} / z_{n}$ for all $x_{n} \in\left[0, z_{n}\right]$ where $n^{*}=n$. This type of dominance coincides with sequential poverty gap dominance where relative poverty gaps instead of absolute ones are used.

The normative implications of these conditions can be derived simply by generalizing the approach considered in the previous sections considering evaluations based on the measure $P($.$) in (1) but modifying the axioms BGP and BGTP in order to$ consider between groups comparisons made at income levels identified in $C$. If we denote these modified versions of the axioms as $\operatorname{BGP}(C)$ and $\operatorname{BGTP}(C)$, then the next proposition follows by simple adjustment of the results in the first part of this paper.

Proposition 11 For a fixed $\mathbf{z} \in Z^{n}$ and a given $C$.

(i) $P(F) \leq P(G)$ for all $u_{i}$ satisfying properties $A, W M$ and $B G P(C)$ if and only if $F \succcurlyeq(C) S D(1)[\mathbf{z}] G$.

(ii) $P(F) \leq P(G)$ for all $u_{i}$ satisfying properties $A$, WM, $B G P(C)$, WPT and $B G T P(C)$ if and only if $F \succcurlyeq_{(C) S D(2)[\mathbf{z}]} G$.

It will then be natural to investigate robust poverty dominance conditions that apply, for a fixed $\mathbf{z} \in Z^{n}$, for all $C^{\prime}$ 's within the set $\mathcal{C}$ of admissible profiles.

Let $\mathcal{C}$ denote the set of all ranked incomes $x_{1} \geq x_{2} \geq \ldots \geq x_{k} \geq \ldots \geq x_{n *} \geq 0$ in groups $i=1,2, . . n^{*}$ for $n^{*} \leq n$ where $x_{i} \in\left[0, z_{i}\right)$ such that $x_{i+1} \leq x_{i} \leq x_{i+1}+$ 
$\delta_{i}$ where $\delta_{i}:=z_{i}-z_{i+1}$. The next proposition identifies the algorithm associated with the sequential stochastic dominance conditions ensuring robustness of poverty comparisons for all $C \in \mathcal{C}$ given a fixed $\mathbf{z} \in Z^{n}$. The result is obtained readjusting the algorithms presented in the previous propositions. We illustrate first its construction for the case of two groups. For any income level $x_{2}$ in Group 2 there exists an income level $x_{1} \in\left[x_{2}, x_{2}+\delta_{1}\right]$ in Group 1 that is comparable to it according to a criterion $C \in \mathcal{C}$. Robustness of the poverty dominance condition requires to check that $(C) S D(j)[\mathbf{z}]$ holds (at the 2nd stage of the sequential procedure) for any pair of $x_{2}$ and $x_{1} \in\left[x_{2}, x_{2}+\delta_{1}\right]$ and for all $x_{2} \in\left[0, z_{2}\right)$. Therefore it is necessary and sufficient to combine $\Delta_{2}^{[j]}\left(x_{2}\right)$ with the maximum value obtained by $\Delta_{1}^{[j]}\left(x_{1}\right)$ within the range $x_{1} \in\left[x_{2}, x_{2}+\delta_{1}\right]$ and check that their sum is not positive for all $x_{2} \in\left[0, z_{2}\right)$.

Once a third group is added to the list, then for any $x_{3} \in\left[0, z_{3}\right)$ there will be a range of values for $x_{2}$ that can be considered comparable to it, this will be the case for $x_{2} \in\left[x_{3}, x_{3}+\delta_{2}\right]$. Dominance should be checked to be robust not only for any pair $x_{3}$ and $x_{2}$ but also, given a value for $x_{2}$, for any set of $x_{1} \in\left[x_{2}, x_{2}+\delta_{1}\right]$ identified at the earlier stage.

For the general case we can summarize the algorithm to check as follows: for $i=0,1,2, . . n-1$ let

$$
\tilde{\Delta}_{i+1}^{*[j]}(x ; \mathbf{z}):=\Delta_{i+1}^{[j]}(x)+\hat{\Delta}_{i}^{*[j]}(x ; \mathbf{z}) \text { for all } x \in\left[0, z_{i+1}\right),
$$

where $\hat{\Delta}_{0}^{*[j]}(x ; \mathbf{z}):=0$ for all $x$, while for $i=1,2, . . n$ we define

$$
\hat{\Delta}_{i}^{*[j]}(x ; \mathbf{z}):=\max _{t \in\left[x ; \min \left\{x+\delta_{i}, z_{i}\right\}\right], t \neq z_{i}}\left\{\tilde{\Delta}_{i}^{*[j]}(t ; \mathbf{z})\right\} .
$$

Proposition 12 Let $\mathbf{z} \in Z^{n}$ and $j=1,2$. The following statements are equivalent:

(i) $F \succcurlyeq_{(C) S D(j)[\mathbf{z}]} G$ for all $C \in \mathcal{C}$.

(ii) $\tilde{\Delta}_{i}^{*[j]}(x ; \mathbf{z}) \leq 0$ for all $x \in\left[0, z_{i}\right)$ and all $i=1,2, \ldots n$.

To complete the analysis we move to consider the case where the above dominance condition is made robust also to changes in poverty lines. In order to compare the results with those in Propositions 5 and 6 obtained for PGD we first analyze the case where $n=2$ and $\mathbf{z} \in Z^{2}\left(\mathbf{z}^{-}, \mathbf{z}^{+}\right)$. We will then move to consider the case where $n \geq 2$ and $\mathbf{z} \in Z^{n}\left(\mathbf{0}, \mathbf{z}^{+}\right)$that is we will consider the set of all positive ranked poverty lines with upper bound $\mathbf{z}^{+}$.

We illustrate dominance conditions first for the two groups case, deriving then the general algorithm by induction. Fix $z_{i}^{+}, z_{i}^{-}$for $i=1,2$, then for any income level $x_{2}$ in Group 2 the comparability set for the associated income levels $x_{1}$ in Group 1 is given by $x_{1} \in\left[x_{2}, x_{2}+z_{1}-z_{2}\right]$. The largest possible set is obtained taking $\sup \left\{z_{1}: z_{1} \in\left[z_{1}^{-}, z_{1}^{+}\right]\right\}=z_{1}^{+}$and $\inf \left\{z_{2}: z_{2} \in\left[\max \left\{x_{2}, z_{2}^{-}\right\}, z_{2}^{+}\right]\right\}$which equals $z_{2}^{-}$if $x_{2}<z_{2}^{-}$and equals $x_{2}$ if $x_{2} \geq z_{2}^{-}$. Thus, at the second stage of comparison necessarily the dominance condition has to be satisfied for all $x_{2} \in\left[0, z_{2}^{-}\right)$and all associated $x_{1} \in\left[x_{2}, x_{2}+z_{1}^{+}-z_{2}^{-}\right]$and for all $x_{2} \in\left[z_{2}^{-}, z_{2}^{+}\right)$and all associated $x_{1} \in\left[x_{2}, z_{1}^{+}\right)$. 
In order to simplify the notation we suggest the extension to the $n$-groups case of the algorithm illustrated for the first two groups obtained as follows: let $\delta_{i}^{*}:=$ $z_{i}^{+}-z_{i+1}^{-} \geq 0$, for $i=0,1,2, . . n-1$ and

$$
\tilde{\Delta}_{i+1}^{*[j]}\left(x ; \mathbf{z}^{-}, \mathbf{z}^{+}\right):=\Delta_{i+1}^{[j]}(x)+\hat{\Delta}_{i}^{*[j]}\left(x ; \mathbf{z}^{-}, \mathbf{z}^{+}\right) \text {for all } x \in\left[0, z_{i+1}^{+}\right),
$$

where $\hat{\Delta}_{0}^{*[j]}\left(x ; \mathbf{z}^{-}, \mathbf{z}^{+}\right):=0$ for all $x$, while for $i=1,2, . . n$ we define

$$
\hat{\Delta}_{i}^{*[j]}\left(x ; \mathbf{z}^{-}, \mathbf{z}^{+}\right):=\max _{t \in\left[x ; \min \left\{x+\delta_{i}^{*}, z_{i}^{+}\right\}\right], t \neq z_{i}^{+}}\left\{\tilde{\Delta}_{i}^{*[j]}\left(t ; \mathbf{z}^{-}, \mathbf{z}^{+}\right)\right\} .
$$

Proposition 13 Let $j=1,2$, and $n=2$. The following statements are equivalent:

(i) $F \succcurlyeq_{(C) S D(j)[\mathbf{z}]} G$ for all $C \in \mathcal{C}$ and all $\mathbf{z} \in Z^{2}\left(\mathbf{z}^{-}, \mathbf{z}^{+}\right)$.

(ii) $\tilde{\Delta}_{i}^{*[j]}\left(x ; \mathbf{z}^{-}, \mathbf{z}^{+}\right) \leq 0$ for all $x \in\left[0, z_{i}^{+}\right)$and all $i=1,2$.

If we consider more than 2 groups the algorithm in (14) and (15) provides a sufficient condition for dominance in the general case where $\mathbf{z} \in Z^{n}\left(\mathbf{z}^{-}, \mathbf{z}^{+}\right)$. The reason can be illustrated just considering the three groups case. Once a third group is added then any $x_{3} \in\left[0, z_{3}^{-}\right)$is associated with a value of $x_{2}$ such that $x_{2} \in$ $\left[x_{3}, x_{3}+z_{2}^{+}-z_{3}^{-}\right]$, which in turns according to the algorithm in (14) and (15) is associated with values of $x_{1} \in\left[x_{2} ; \min \left\{x_{2}+z_{1}^{+}-z_{2}^{-}, z_{1}^{+}\right\}\right], x_{1} \neq z_{1}^{+}$. But the choice of $x_{2}$ as an admissible comparable value at the second stage will implicitly constrain the values of the associated admissible poverty lines for the second group. In particular, if $z_{2}^{-}-x_{2}<z_{3}^{-}-x_{3}$ then the lowest possible value for the poverty line $z_{2}$ that can be consistent with $x_{2}$ being an admissible value comparable to $x_{3}$ is given by $z_{2}^{*}=$ $x_{2}+z_{3}^{-}-x_{3}>z_{2}^{-}$. This value is obtained requiring that the poverty gap associated with $x_{2}$ (i.e. $z_{2}-x_{2}$ ) is at least as large as the minimum poverty gap associated with $x_{3}$ (i.e. $z_{3}^{-}-x_{3}$ ). The values of $x_{1}$ that are comparable to this value of $x_{2}>x_{3}+z_{2}^{-}-z_{3}^{-}$ are therefore obtained substituting $z_{2}^{*}$ for $z_{2}^{-}$in $\left[x_{2} ; \min \left\{x_{2}+z_{1}^{+}-z_{2}^{-}, z_{1}^{+}\right\}\right]$, that is are all values $x_{1} \in\left[x_{2} ; \min \left\{x_{3}+z_{1}^{+}-z_{3}^{-}, z_{1}^{+}\right\}\right], x_{1} \neq z_{1}^{+}$. Given the constraint $x_{2}>x_{3}+z_{2}^{-}-z_{3}^{-}$this range of values is narrower than the range identified by $x_{1} \in\left[x_{2} ; \min \left\{x_{2}+z_{1}^{+}-z_{2}^{-}, z_{1}^{+}\right\}\right], x_{1} \neq z_{1}^{+}$as in (15), therefore the algorithm provides more stringent conditions than those needed.

A general algorithm implementing necessary and sufficient conditions for dominance that are valid for all $\mathbf{z} \in Z^{n}\left(\mathbf{z}^{-}, \mathbf{z}^{+}\right)$may turn out to be cumbersome. However notice that when $\mathbf{z}^{-}=\mathbf{0}$ the previous issue does not arise. In that case $x_{3} \in\left[z_{3}^{-}, z_{3}^{+}\right)$, as a result the set of comparable values for $x_{2}$ will be given by all $x_{2} \in\left[x_{3}, z_{2}^{+}\right)$. These values are obtained by the algorithm in (14) and (15) under the assumption $\mathbf{z}^{-}=\mathbf{0}$ which implies that $\min \left\{x+\delta_{i}^{*}, z_{i}^{+}\right\}=z_{i}^{+}$for all $x \geq 0$ in (15). Thus the above algorithm will provide necessary and sufficient conditions for dominance for all ranked poverty line vectors in $Z^{n}\left(\mathbf{0}, \mathbf{z}^{+}\right)$.

Proposition 14 Let $j=1,2$. The following statements are equivalent:

(i) $F \succcurlyeq_{(C) S D(j)[\mathbf{z}]} G$ for all $C \in \mathcal{C}$ and all $\mathbf{z} \in Z^{n}\left(\mathbf{0}, \mathbf{z}^{+}\right)$.

(ii) $\tilde{\Delta}_{i}^{*[j]}\left(x ; \mathbf{0}, \mathbf{z}^{+}\right) \leq 0$ for all $x \in\left[0, z_{i}^{+}\right)$and all $i=1,2, \ldots n$. 
Note that $\hat{\Delta}_{i}^{*[j]}\left(x ; \mathbf{0}, \mathbf{z}^{+}\right)$is obtained as $\max _{t \in\left[x ; z_{i}^{+}\right)}\left\{\tilde{\Delta}_{i}^{*[j]}\left(t ; \mathbf{0}, \mathbf{z}^{+}\right)\right\}$. Thus $\tilde{\Delta}_{i}^{*[j]}\left(x ; \mathbf{0}, \mathbf{z}^{+}\right)$ coincides with $\tilde{\Delta}_{i}^{[j]}\left(x ; \mathbf{0}, \mathbf{z}^{+}\right)$in (7) and (8) leading to the same dominance condition as in Proposition 6 with the obvious implication that the Bourguignon (1989) algorithm follows as a special case once $\mathbf{z}^{+}=\bar{z} \mathbf{1}$.

A comparison with the result in Proposition 6 makes evident that the dominance conditions $(C) S D(j)[\mathbf{z}]$ that are satisfied for all $C \in \mathcal{C}$ and dominance conditions $S P G D(j)[\mathbf{z}]$ are equivalent when are required to hold for all $\mathbf{z} \in Z^{n}\left(\mathbf{0}, \mathbf{z}^{+}\right)$. Note that for a fixed $\mathbf{z} \in Z^{n}$ the conditions $S P G D(j)[\mathbf{z}]$ and $S P D(j)[\mathbf{z}]$ are obtained as extreme cases of $(C) S D(j)[\mathbf{z}]$ when $C$ admits respectively between groups comparability at fixed poverty gap levels or at fixed income levels. When dominance is checked for all $\mathbf{z} \in Z^{n}\left(\mathbf{0}, \mathbf{z}^{+}\right)$then $S P G D(j)[\mathbf{z}]$ is consistent with a larger class of dominance conditions based on different comparability hypothesis including $S P D(j)[\mathbf{z}]$. However as pointed out in the previous sections $S P D(j)[\mathbf{z}]$ for all $\mathbf{z} \in Z^{n}\left(\mathbf{0}, \mathbf{z}^{+}\right)$does not imply $S P G D(j)[\mathbf{z}]$ for all $\mathbf{z} \in Z^{n}\left(\mathbf{0}, \mathbf{z}^{+}\right)$as a result:

Corollary 4 Let $j=1$, 2. $F \succcurlyeq_{S P G D(j)[\mathbf{z}]} G$ for all $\mathbf{z} \in Z^{n}\left(\mathbf{0}, \mathbf{z}^{+}\right) \Longleftrightarrow F \succcurlyeq_{(C) S D(j)[\mathbf{z}]} G$

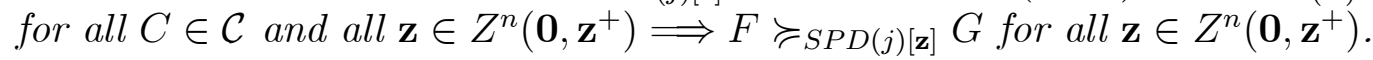

\section{Conclusions}

In what respects do our results differ from the standard sequential dominance conditions derived following the approach suggested in Atkinson (1992)?

Poverty evaluations differ from inequality and welfare evaluations in one significant aspect, the existence of a threshold, the poverty line. While in general the income distributions compared for welfare and inequality evaluations are unbounded, or bounded at an arbitrary maximum, for poverty the distributions are censored at the poverty line. This process creates a reference point, which is an alternative to the origin or zero income point.

It is therefore possible to build up normative evaluation models in which comparisons are made taking distances from this reference point (see statement B in the Introduction) and not only from the origin to be ethically relevant (see statement A in the Introduction). This is the case in our model, which focuses upon poverty gaps and not incomes. When we consider homogeneous populations, once the poverty line is fixed the two perspectives coincide, but for the purpose of making between-group poverty comparisons, when poverty lines could differ for the different groups, then, as we have plainly demonstrated here, choosing incomes or poverty gaps as the relevant indicator makes a difference.

Our results have shown that APGP curves (as well as poverty-gap distributions) have a key role in making sequential poverty comparisons. In particular, the proportion of poor individuals in the society or their average poverty gap play a role in the comparisons that was neglected in the existing poverty dominance criteria for heterogeneous populations. 
Acknowledgment: Earlier versions of this paper has been presented at the conferences: "Income Distribution and Welfare" Bocconi University, Milan, May 2002; Journées d'Economie Publique Louis-André Gérard-Varet, Marseille, May 2003; Public Economic Theory, Duke, June 2003; CHILD workshop, Garda, May 2004. We are grateful to conference participants, Russel Davidson and Krishna Pendakur for helpful comments. Zoli acknowledges hospitality of the Department of Quantitative Methods, at Bocconi University, Milan where he was visiting during part of the revision of the paper.

\section{References}

[1] Asplund, E. and Bungart, L. (1966): A First Course in Integration. New York: Holt, Rinehart and Winston.

[2] Atkinson, A. B. (1970): On the measurement of inequality. Journal of Economic Theory, 2, 244-263.

[3] Atkinson, A. B. (1987): On the measurement of poverty. Econometrica 55, 749764.

[4] Atkinson, A. B. (1992): Measuring poverty and differences in family composition. Economica 59, 1-16.

[5] Atkinson, A. B. and Bourguignon, F. (1987): Income distribution and differences in needs. In Arrow and the Foundations of the Theory of Economic Policy, Feiwel (ed.), 350-370. London: Macmillan.

[6] Bazen, S. and Moyes, P. (2003): International comparisons of income distributions when population structures differ. In Research in Economic Inequality, Volume 9, 85-104.

[7] Bourguignon, F. (1989): Family size and social utility: income distribution dominance criteria. Journal of Econometrics, 42, 67-80.

[8] Bourguignon, F. and Fields, G. (1997): Discontinuous losses from poverty, generalized $\mathrm{P}$ measures and optimal transfers to the poor. Journal of Public Economics, 63, 155-175.

[9] Castagnoli, E. and Maccheroni, F. (1998): Generalized stochastic dominance and unanimous preferences. In Generalized Convexity and Optimization for Economic and Financial Decisions, Giorgi, G. and Rossi, F. (eds.), 111-120. Bologna: Pitagora.

[10] Chakravarty, S. R. (1983): Ethically flexible measures of poverty. Canadian Journal of Economics, 16, 74-85. 
[11] Chakravarty, S. R. (1983a): Measures of poverty based on the representative income gap. Sankya, 45B.

[12] Chambaz, C. and Maurin, E. (1998): Atkinson and Bourguignon's dominance criteria: extended and applied to the measurement of poverty in France. Review of Income $\&$ Wealth, 44, 497-515.

[13] Davidson, R. and Duclos, J. Y. (2000): Statistical inference for stochastic dominance and for the measurement of poverty and inequality, Econometrica 68, $1435-1465$.

[14] Donaldson, D. and Pendakur, K. (2004): Equivalent-expenditure functions and expenditure-dependent equivalence scales. Journal of Public Economics, 88, 175208.

[15] Duclos, J. Y. and Makdissi P. (1999): Sequential stochastic dominance and the robustness of poverty orderings, Cahier de recherche 99-05, CRÉFA, Département d'économie, Université Laval.

[16] Duclos, J. Y., Sahn, D. and Younger, S. D. (2003): Robust multidimensional poverty comparisons. Cahier de recherche 03-04, CIRPÉE, Université Laval.

[17] Ebert, U. and Moyes, P. (2003): Equivalence scales reexamined. Econometrica, 71, 319-343.

[18] Fleurbaey, M., Hagneré, C. and Trannoy, A. (2003): Welfare comparisons with bounded equivalence scales. Journal of Economic Theory, 110, 309-336.

[19] Foster, J.; Greer, J. and Thorbecke, D. (1984): A class of decomposable poverty measures. Econometrica, 52, 761-766.

[20] Foster, J. and Shorrocks, A. F. (1988a): Poverty orderings. Econometrica, 56, 173-177.

[21] Foster, J. and Shorrocks, A. F. (1988b): Poverty orderings and welfare dominance. Social Choice and Welfare, 5, 179-198.

[22] Foster, J. and Shorrocks, A. F. (1988c): Inequality and poverty orderings. European Economic Review, 32, 654-662.

[23] Jenkins, S. and Lambert, P. J. (1993): Ranking income distributions when needs differ. Review of Income \& Wealth 39, 337-356.

[24] Jenkins, S. and Lambert, P. J. (1997): Three I's of poverty curves, with an analysis of UK poverty trends. Oxford Economic Papers 49, 317-327. 
[25] Lambert, P. and Ramos, X. (2002): Welfare comparisons: sequential procedures for heterogeneous populations. Economica 69, 549-562.

[26] Müller, A. (1997): Stochastic orders generated by integrals: a unified study. Advances in Applied Probability, 29, 414-428.

[27] Saposnik, R. (1981): Rank-dominance in income distributions. Public Choice, 36, $147-51$.

[28] Sen, A. K. (1976): Poverty: an ordinal approach to measurement. Econometrica, 44, 219-231.

[29] Shorrocks, A. F. (1995): Revisiting the Sen poverty index. Econometrica, 63, 1225-1230.

[30] Shorrocks, A. F. (1998): Deprivation profiles and deprivation indices. In The Distribution of Welfare and Household Production: International Perspectives, Jenkins, S. A., Kaptein, S. A. and van Praag, B. eds. 250-267. London: Cambridge University Press.

[31] Spencer, B. D. and Fisher, S. (1992): On comparing distributions of poverty gaps. Sankya, 54B, 114-126.

[32] Thistle, P. (1989): Ranking Distributions with generalized Lorenz curves. Southern Economics Journal, 56, 1-12.

[33] Zheng, B. (1997): Aggregate poverty measures. Journal of Economic Surveys, 11, 123-162.

[34] Zheng, B. (1999): On the power of poverty orderings. Social Choice and Welfare, 16, 349-371.

[35] Zheng, B. (2000): Poverty orderings. Journal of Economic Surveys, 14, 427-466.

[36] Zoli, C. (2000):Inverse sequential stochastic dominance: rank-dependent welfare, deprivation and poverty measurement. University of Nottingham, Discussion Paper in Economics 00/11. This paper is available at: http://www.nottingham.ac.uk/economics/research/dp/school_dp/dp.00.11.pdf. 


\section{Appendix}

Claim $1 P($.$) satisfies W M$ and $B G P$ if and only if $u_{i}(\gamma)$ satisfies $A 1$.

Proof. $P($.$) satisfies WM iff it is non-decreasing w.r.t. \gamma$ that is iff $u_{i}^{\prime}(\gamma) \geq 0$ for all $\gamma>0$, and all $i=1,2, . . n$. For $F$ and $F^{\prime}$ obtained as in the definition of BGP the change in poverty evaluated according to $P($.$) is$

$$
P(F)-P(G)=q\left[u_{i}(\gamma+\delta)-u_{i}(\gamma)\right], P\left(F^{\prime}\right)-P(G)=q\left[u_{j}(\gamma+\delta)-u_{j}(\gamma)\right] .
$$

Thus we get

$$
P(F)-P\left(F^{\prime}\right) \geq 0 \Longleftrightarrow u_{i}(\gamma+\delta)-u_{i}(\gamma) \geq u_{j}(\gamma+\delta)-u_{j}(\gamma)
$$

for all $\gamma>0$, all $\delta>0$, all $j>i$. Dividing both sides by $\delta>0$ we obtain

$$
\frac{u_{i}(\gamma+\delta)-u_{i}(\gamma)}{\delta} \geq \frac{u_{j}(\gamma+\delta)-u_{j}(\gamma)}{\delta}
$$

letting $\delta \rightarrow 0$ we have $u_{i}^{\prime}(\gamma) \geq u_{j}^{\prime}(\gamma)$ for all $\gamma>0$, all $j>i$, which implies that (16) is satisfied for all $\delta>0$ and all $\gamma>0$, i.e. BGP is satisfied. Combining with $u_{i}^{\prime}(\gamma) \geq 0$ for all $\gamma>0$ and all $i=1,2, . . n$, we get property A1.

Claim $2 P($.$) satisfies WPT and BGTP if and only if u_{i}(\gamma)$ satisfies A2.

Proof. We first note that for all $\gamma>\delta, \varepsilon>0, \delta>0$ and all $j>i$, the effect on $P($. of a regressive transfer occurring in group $i$ is

$$
\Delta P=q\left[u_{i}(\gamma-\delta)-u_{i}(\gamma)\right]+q\left[u_{i}(\gamma+\varepsilon+\delta)-u_{i}(\gamma+\varepsilon)\right] .
$$

Therefore we get

$$
\Delta P \geq 0 \Longleftrightarrow u_{i}(\gamma+\varepsilon+\delta)-u_{i}(\gamma+\varepsilon) \geq u_{i}(\gamma)-u_{i}(\gamma-\delta)
$$

for all $\gamma>\delta$, all $\delta>0, \varepsilon>0$ and all $i$. Dividing both sides by $\delta>0$ and letting $\delta \rightarrow 0$ we obtain

$$
u_{i}^{\prime}(\gamma+\varepsilon) \geq u_{i}^{\prime}(\gamma)
$$

for all $\gamma>0, \varepsilon>0$. That is $u_{i}^{\prime \prime}(\gamma) \geq 0$. This condition implies that (17) is satisfied for all $\gamma>0$ and all $\delta>0$, i.e. WPT is satisfied.

According to BGTP the comparison of the marginal impact of the regressive transfers in groups $i$ and $j>i$ requires that

$$
\delta u_{i}^{\prime}(\gamma)-\delta u_{i}^{\prime}(\gamma+\varepsilon) \leq \delta u_{j}^{\prime}(\gamma)-\delta u_{j}^{\prime}(\gamma+\varepsilon) \text { where } j>i
$$

It follows that for all $\gamma>0, \varepsilon>0$,

$$
\frac{u_{i}^{\prime}(\gamma+\varepsilon)-u_{i}^{\prime}(\gamma)}{\varepsilon} \geq \frac{u_{j}^{\prime}(\gamma+\varepsilon)-u_{j}^{\prime}(\gamma)}{\varepsilon} \geq 0 \text { where } j>i
$$


Taking the limit as $\varepsilon \rightarrow 0$, we get the first part of property A2.

Note that $u_{i}(\gamma+\varepsilon+\delta)-u_{i}(\gamma+\varepsilon)-\left[u_{i}(\gamma)-u_{i}(\gamma-\delta)\right]=\int_{\gamma}^{\gamma+\delta}\left(\int_{\tau}^{\tau+\varepsilon} u_{i}^{\prime \prime}(t) d t\right) d \tau$ it follows that $u_{i}^{\prime \prime}(\gamma) \geq u_{j}^{\prime \prime}(\gamma) \geq 0$ implies that $\int_{\gamma}^{\gamma+\delta}\left(\int_{\tau}^{\tau+\varepsilon} u_{i}^{\prime \prime}(t) d t\right) d \tau \geq \int_{\gamma}^{\gamma+\delta}\left(\int_{\tau}^{\tau+\varepsilon} u_{j}^{\prime \prime}(t) d t\right) d \tau \geq$ 0 as required by WPT and BGTP.

Claim 3 If $P($.$) satisfies A, A 0$ and $W M$ then $p_{i}\left(x, z_{i}\right) \geq p_{i+1}\left(x, z_{i+1}\right) \geq 0$ for all $i=1,2, . . n-1$ and for all $x$ and $p_{i}\left(x, z_{i}\right) \geq p_{i}\left(x^{\prime}, z_{i}\right)$ where $x<x^{\prime}$ for all $i=1,2$,..n.

Proof. Recall that $u_{i}(\gamma):=p_{i}\left(z_{i}-\gamma, z_{i}\right)$. Note that A and WM are equivalent when applied considering either $u_{i}(\gamma)$ or $p_{i}\left(x, z_{i}\right)$. According to A0, $u_{i}(\gamma) \geq u_{i+1}(\gamma) \geq 0$ for all $\gamma>0$, and all $i=1,2, . . n-1$, that is $p_{i}\left(z_{i}-\gamma, z_{i}\right) \geq p_{i+1}\left(z_{i+1}-\gamma, z_{i+1}\right) \geq 0$ for all $\gamma>0$, and all $i=1,2, . . n-1$. While according to WM $u_{i}^{\prime}(\gamma) \geq 0$ for all $\gamma>0$, and all $i=1,2, . . n$. That is $p_{i}^{\prime}\left(z_{i}-\gamma, z_{i}\right) \leq 0$ for all $\gamma>0$, and all $i=1,2, . . n$, where $p_{i}^{\prime}\left(x, z_{i}\right)$ denotes the partial derivative of $p_{i}\left(x, z_{i}\right)$ w.r.t. $x$. This condition implies that $p_{i}\left(z_{i}-\gamma, z_{i}\right) \leq p_{i}\left(z_{i}-\gamma^{\prime}, z_{i}\right)$ for all $\gamma^{\prime} \geq \gamma$, all $z_{i}$ and all $i=1,2, . . n$.

Letting $x:=z_{i}-\gamma$ the previous conditions can be restated as $p_{i}\left(x, z_{i}\right) \geq p_{i+1}\left(z_{i+1}-\right.$ $\left.z_{i}+x, z_{i+1}\right) \geq 0$ for all $x>0$, and $p_{i}\left(x^{\prime}, z_{i}\right) \geq p_{i}\left(x, z_{i}\right)$ for all $x^{\prime} \leq x$, all $z_{i}$ and all $i=1,2, . . n$. Given that $z_{i} \geq z_{i+1}$ then we can substitute $z_{i+1}-z_{i}+x$ for $x^{\prime}$ noting that $x^{\prime} \leq x$.

Putting together the two conditions, we have $p_{i}\left(x, z_{i}\right) \geq p_{i+1}\left(x^{\prime}, z_{i+1}\right) \geq p_{i+1}\left(x, z_{i+1}\right) \geq$ 0 for all $x$,all $z_{i} \geq z_{i+1}$ and all $i=1,2, . . n-1$. This is the equivalent of A0 for comparisons made at income levels.

Note that the converse of the claim is not true. Consider two groups $(i=1,2)$ with $z_{1}>z_{2}$. Let $p_{i}\left(x, z_{i}\right)=\left(z_{2}-x\right)$ if $x \leq z_{2}$ and $p_{i}\left(x, z_{i}\right)=0$ if $x>z_{2}$ for $i=1,2$. Note that $p_{1}\left(x, z_{1}\right)=p_{2}\left(x, z_{2}\right) \geq 0$ but for all values of $\gamma \in\left(0, z_{2}\right]$ we have $u_{1}(\gamma)<u_{2}(\gamma)$ thereby violating A0.

Claim 4 If $P($.$) satisfies A, A 1$ and WPT then $p_{i}^{\prime}\left(x, z_{i}\right) \leq p_{i+1}^{\prime}\left(x, z_{i+1}\right) \leq 0$ for all $i=1,2, . . n-1$ and for all $x$.

Proof. Recall that $u_{i}(\gamma):=p_{i}\left(z_{i}-\gamma, z_{i}\right)$. According to A1, $u_{i}^{\prime}(\gamma) \geq u_{i+1}^{\prime}(\gamma) \geq 0$ for all $\gamma>0$, and all $i=1,2, . . n-1$, that is $p_{i}^{\prime}\left(z_{i}-\gamma, z_{i}\right) \leq p_{i+1}^{\prime}\left(z_{i+1}-\gamma, z_{i+1}\right) \leq 0$ for all $\gamma>0$, and all $i=1,2, . . n-1$. While according to WPT $u_{i}^{\prime \prime}(\gamma) \geq 0$ for all $\gamma>0$, and all $i=1,2, . . n$. That is $p_{i}^{\prime \prime}\left(z_{i}-\gamma, z_{i}\right) \geq 0$ for all $\gamma>0$, and all $i=1,2, . . n$, implying that $p_{i}^{\prime}\left(z_{i}-\gamma, z_{i}\right) \geq p_{i}^{\prime}\left(z_{i}-\gamma^{\prime}, z_{i}\right)$ for all $\gamma^{\prime} \geq \gamma$, all $z_{i}$ and all $i=1,2, . . n$.

Letting $x:=z_{i}-\gamma$ the previous conditions can be restated as $p_{i}^{\prime}\left(x, z_{i}\right) \leq p_{i+1}^{\prime}\left(z_{i+1}-\right.$ $\left.z_{i}+x, z_{i+1}\right) \leq 0$ for all $x>0$, and $p_{i}^{\prime}\left(x, z_{i}\right) \geq p_{i}^{\prime}\left(x^{\prime}, z_{i}\right)$ for all $x^{\prime} \leq x$, all $z_{i}$ and all $i=1,2, . . n$. Given that $z_{i} \geq z_{i+1}$ then we can substitute $z_{i+1}-z_{i}+x$ for $x^{\prime}$ noting that $x^{\prime} \leq x$. Putting together the two conditions, we have $p_{i}^{\prime}\left(x, z_{i}\right) \leq p_{i+1}^{\prime}\left(x^{\prime}, z_{i+1}\right) \leq$ $p_{i+1}^{\prime}\left(x, z_{i+1}\right)$ for all $x$, all $z_{i} \geq z_{i+1}$ and all $i=1,2, . . n-1$.

Note that the converse of the claim is not true. Let $p_{j}\left(x, z_{i}\right)=\left(z_{j}-x\right)^{2}$ if $z_{j} \geq x$, otherwise $p_{j}\left(x, z_{i}\right)=0$ for $j=i, i+1$. Then $p_{i}^{\prime}\left(x, z_{i}\right)=-2\left(z_{i}-x\right)<$ 
$-2\left(z_{i+1}-x\right)=p_{i+1}^{\prime}\left(x, z_{i}\right)$ if $z_{i+1} \geq x$, otherwise $p_{i+1}^{\prime}\left(x, z_{i}\right)=0$ and the condition $p_{i}^{\prime}\left(x, z_{i}\right) \leq p_{i+1}^{\prime}\left(x, z_{i+1}\right)$ is still satisfied. Note that A1 is satisfied with equality, i.e. $u_{i}^{\prime}(\gamma)=u_{i+1}^{\prime}(\gamma)=-2$. It is possible to modify slightly $p_{i}\left(x, z_{i}\right)$ for values of $x \in\left(z_{i+1}, z_{i}\right)$ such that $u_{i}^{\prime}(\gamma)>u_{i+1}^{\prime}(\gamma)$ while the condition $p_{i}^{\prime}\left(x, z_{i}\right) \leq p_{i+1}^{\prime}\left(x, z_{i+1}\right)$ is unaffected given that $p_{i+1}^{\prime}\left(x, z_{i}\right)=0$ for all $x \in\left(z_{i+1}, z_{i}\right)$.

Claim 5 For a fixed $\mathbf{z} \in Z^{n}, P(F) \leq P(G)$ for all $u_{i}$ satisfying $A$ and $A 1$ if and only if $F \succcurlyeq_{R B D(1)[\mathbf{z}]} G$.

Proof. We show only the sufficiency part. The necessity part can be found by rearranging the proofs in Chambaz and Maurin (1998) or Zoli (2000).

Integrating by parts in (4), we have:

$$
\begin{aligned}
\Delta P= & \sum_{i=1}^{n} \int_{0}^{z_{i}} u_{i}^{\prime}\left(z_{i}-x\right)\left[q_{i}^{F} F_{i}(x)-q_{i}^{G} G_{i}(x)\right] d x \\
& +\sum_{i=1}^{n}\left[u_{i}\left(z_{i}-x\right)\left[q_{i}^{F} F_{i}(x)-q_{i}^{G} G_{i}(x)\right]\right]_{0}^{z_{i}} .
\end{aligned}
$$

where $u_{i}^{\prime}$ denotes derivative w.r.t. $z_{i}-x$. The second term sums to zero given that $u_{i}(0)=0$ for all $i$, therefore

$$
\Delta P=\sum_{i=1}^{n} \int_{0}^{z_{i}} u_{i}^{\prime}\left(z_{i}-x\right)\left[q_{i}^{F} F_{i}(x)-q_{i}^{G} G_{i}(x)\right] d x .
$$

Substituting $x=z_{i}-\gamma$, we get

$$
\Delta P=\sum_{i=1}^{n} \int_{0}^{z_{i}} u_{i}^{\prime}(\gamma)\left[\phi_{i}^{F}\left(\gamma, z_{i}\right)-\phi_{i}^{G}\left(\gamma, z_{i}\right)\right] d \gamma .
$$

For all $u_{i}(\gamma)$ satisfying A1, $u_{i}^{\prime}(\gamma)$ can be equivalently defined in terms of functions $\omega_{j}(\gamma)$ for $j=i, \ldots n$, as

$$
u_{i}^{\prime}(\gamma)=\sum_{j=i}^{n} \omega_{j}(\gamma) \text { where } \omega_{j}(\gamma) \geq 0 \text { for all } \gamma \geq 0
$$

Substituting, we have

$$
\Delta P=\sum_{i=1}^{n} \int_{0}^{z_{i}} \sum_{j=i}^{n} \omega_{j}(\gamma)\left[\phi_{i}^{F}\left(\gamma, z_{i}\right)-\phi_{i}^{G}\left(\gamma, z_{i}\right)\right] d \gamma .
$$

Let $\gamma^{*}$ be the highest poverty gap level in all subgroups. After rearranging we get

$$
\Delta P=\int_{0}^{\gamma^{*}} \sum_{k=1}^{n} \omega_{k}(\gamma) \sum_{j=1}^{k}\left[\phi_{j}^{F}\left(\gamma, z_{j}\right)-\phi_{j}^{G}\left(\gamma, z_{j}\right)\right] d \gamma
$$


It follows that, since $\omega_{k}(\gamma) \geq 0$ for all $k=1,2, . . n$ and all $\gamma>0$, the condition

$$
\sum_{j=1}^{k}\left[\phi_{j}^{F}\left(\gamma, z_{j}\right)-\phi_{j}^{G}\left(\gamma, z_{j}\right)\right] \leq 0
$$

for all $i=1,2, . . n$, and for all $\gamma>0$ is sufficient for poverty dominance.

Example 3 Consider two groups $i=1,2$, non-negative incomes, and distribution functions defined as follows: ${ }^{10} F_{1}(x)=0$ and $F_{2}(x)=x \cdot 2 \alpha$ for all $x<1 /(2 \alpha)$, and $F_{1}(x)=F_{2}(x)=1$ for all $x \geq 1 /(2 \alpha) ; G_{1}(x)=G_{2}(x)=x \cdot \beta$ for all $x<1 / \beta$ and $G_{1}(x)=G_{2}(x)=1$ for all $x \geq 1 / \beta$ where $2 \alpha>\beta>0$ and $1 /(2 \alpha)>z_{1}$. Suppose further that $q_{i}^{F}=q_{i}^{G}=1 / 2$ and the poverty lines are $z_{2}=z, z_{1}=c \cdot z$ where $c \geq 1$.
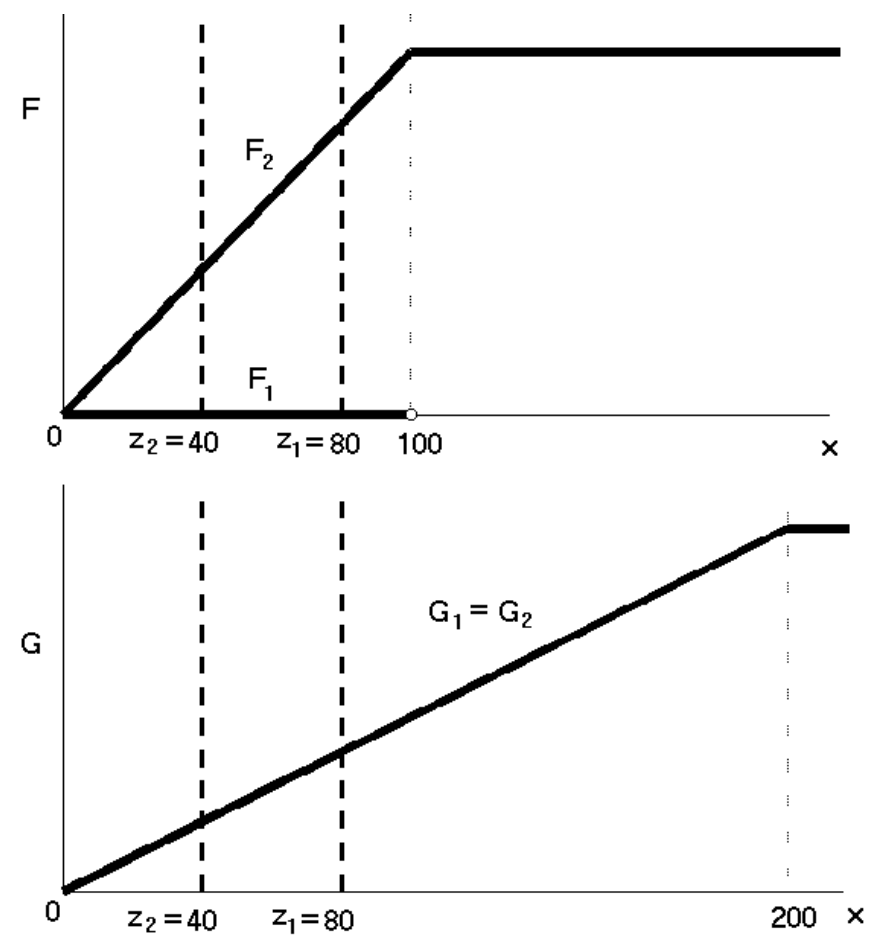

Fig. A1: Ex. $\alpha=\beta=1 / 200, z_{2}=40, z_{1}=80$.

In order to compute the reference-based dominance condition we evaluate $\phi_{i}^{F}\left(\gamma, z_{i}\right)=$ $\frac{1}{2} \cdot F_{i}\left(z_{i}-\gamma\right)$ that is $\phi_{1}^{F}\left(\gamma, z_{1}\right)=0$ for all $\gamma \geq 0, \phi_{2}^{F}\left(\gamma, z_{2}\right)=\left(z_{2}-\gamma\right) \alpha$ for all $\gamma \in\left[0, z_{2}\right]$ and $\phi_{i}^{G}\left(\gamma, z_{i}\right)=\frac{1}{2}\left(z_{i}-\gamma\right) \beta$ for all $\gamma \in\left[0, z_{i}\right], i=1,2$.

The standard (SPD) and reference-based poverty dominance conditions coincide at the first stage: $F$ dominates $G$ given either $F_{1}(x) \leq G_{1}(x)$ for all $x \leq z_{1}$ or $\phi_{1}^{F}\left(\gamma, z_{1}\right) \leq \phi_{1}^{G}\left(\gamma, z_{1}\right)$ for all $\gamma \geq 0$. But at the second stage, the conditions do not coincide. We have $\frac{1}{2} F_{1}(x)+\frac{1}{2} F_{2}(x)=\alpha x$ and $\frac{1}{2} G_{1}(x)+\frac{1}{2} G_{2}(x)=G(x)=x \cdot \beta$. Hence, if $\beta<\alpha$, the second stage of standard dominance (SPD) fails. For the reference-based

\footnotetext{
${ }^{10}$ For simplicity of exposition we consider both continuous and discrete sub-group distributions.
} 
comparison we have $\phi_{1}^{F}\left(\gamma, z_{1}\right)+\phi_{2}^{F}\left(\gamma, z_{2}\right)=\left(z_{2}-\gamma\right) \alpha=(z-\gamma) \alpha$, and $\phi_{1}^{G}\left(\gamma, z_{1}\right)+$ $\phi_{2}^{G}\left(\gamma, z_{2}\right)=\frac{1}{2} \beta\left(z_{1}-\gamma\right)+\frac{1}{2} \beta\left(z_{2}-\gamma\right)=\frac{c+1}{2} \beta z-\beta \gamma$. Suppose that $\beta=\varepsilon \alpha$ where $0<\varepsilon<2$. Then

$$
\begin{aligned}
\phi_{1}^{F}\left(\gamma, z_{1}\right)+\phi_{2}^{F}\left(\gamma, z_{2}\right) & \leq \phi_{1}^{G}\left(\gamma, z_{1}\right)+\phi_{2}^{G}\left(\gamma, z_{2}\right) \\
& \Leftrightarrow(z-\gamma) \alpha \leq \varepsilon \alpha(c+1) z / 2-\varepsilon \alpha \gamma
\end{aligned}
$$

That is, $z \alpha \leq \varepsilon \alpha(c+1) z / 2+(1-\varepsilon) \alpha \gamma$ for all $\gamma$, which requires that $z \alpha \leq \varepsilon \alpha(c+1) z / 2$. Hence

$$
1 \leq \varepsilon \frac{c+1}{2} \Leftrightarrow c \geq \frac{2-\varepsilon}{\varepsilon}
$$

Therefore dominance is satisfied for the reference-based procedure if $c \geq \frac{2-\varepsilon}{\varepsilon}$
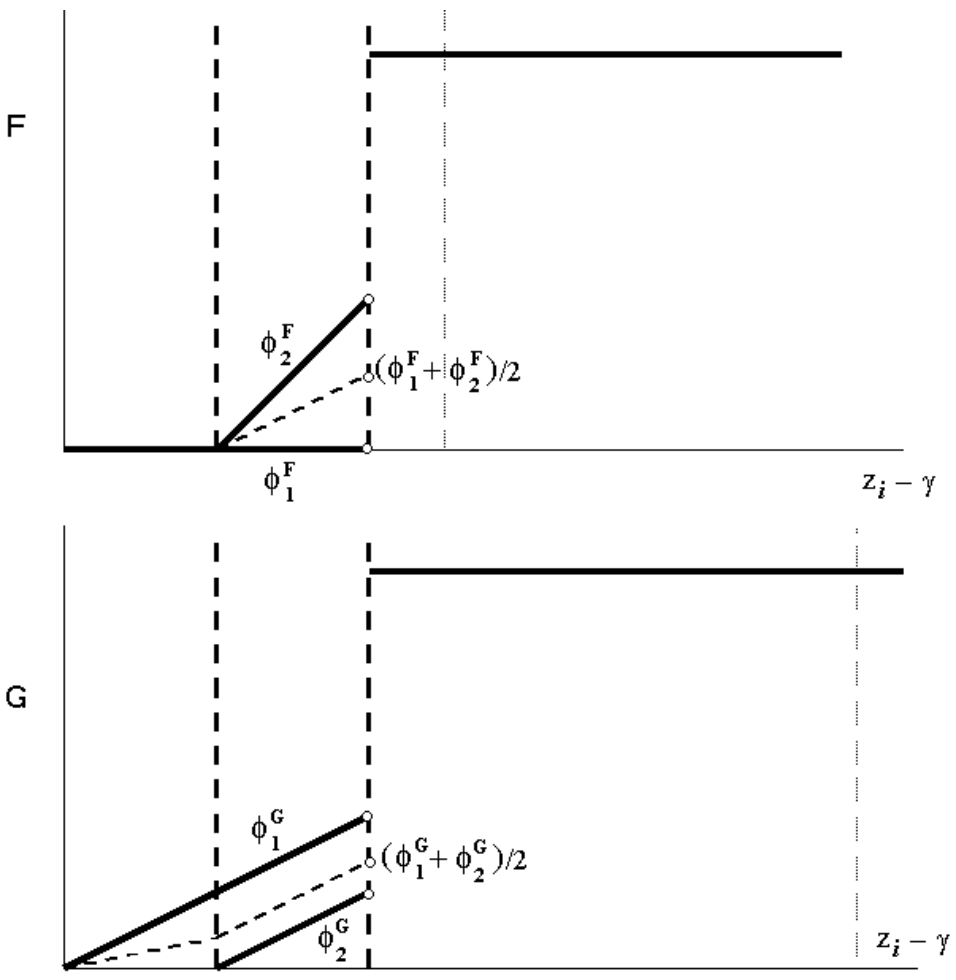

Fig A2: $1^{\text {st }}$ order sequential reference-based dominance

In the above figure we show graphically the dominance comparisons for the same example as in Fig. A1. In this case we get $c=2$, and $\varepsilon=1$. The second stage of the reference-based procedure is checked comparing the dotted lines for both distributions. The dotted line associated with $F$ is below that of $G$, that is $F$ dominates $G$.

Claim 6 For a fixed $\mathbf{z} \in Z^{n}, P(F) \leq P(G)$ for all $u_{i}$ satisfying $A, A 1$ and $A 2$ if and only if $F \succcurlyeq_{R B D(2)[\mathbf{z}]} G$. 
Proof. Again we prove only the sufficiency part (see the proof of Claim 5). For all $u_{i}($.$) satisfying A1 and A2 we have$

$$
u_{i}^{\prime \prime}(\gamma)=\sum_{j=i}^{n} \omega_{j}^{\prime}(\gamma) \text { where } \omega_{j}^{\prime}(\gamma) \geq 0 \text { for all } \gamma>0
$$

Integrating by parts in (22),

$$
\begin{aligned}
\Delta P= & -\int_{0}^{\gamma^{*}} \sum_{k=1}^{n} \omega_{k}^{\prime}(\gamma) \sum_{j=1}^{k}\left(\int_{0}^{\gamma}\left[\phi_{j}^{F}\left(t, z_{j}\right)-\phi_{j}^{G}\left(t, z_{j}\right)\right] d t\right) d \gamma \\
& +\sum_{k=1}^{n} \omega_{k}\left(\gamma^{*}\right)\left(\sum_{j=1}^{k} \int_{0}^{\gamma^{*}}\left[\phi_{j}^{F}\left(t, z_{j}\right)-\phi_{j}^{G}\left(t, z_{j}\right)\right] d t\right) .
\end{aligned}
$$

Noticing that

$$
\omega_{k}\left(\gamma^{*}\right)=\int_{0}^{\gamma^{*}} \omega_{k}^{\prime}(t) d t+\omega_{k}(0)
$$

and substituting, we get, after simplification,

$$
\begin{aligned}
\Delta P= & \sum_{k=1}^{n} \int_{0}^{\gamma^{*}} \omega_{k}^{\prime}(\gamma) \sum_{j=1}^{k}\left(\int_{\gamma}^{\gamma^{*}}\left[\phi_{j}^{F}\left(t, z_{j}\right)-\phi_{j}^{G}\left(t, z_{j}\right)\right] d t\right) d \gamma \\
& +\omega_{k}(0)\left(\sum_{j=1}^{k} \int_{0}^{\gamma^{*}}\left[\phi_{j}^{F}\left(t, z_{j}\right)-\phi_{j}^{G}\left(t, z_{j}\right)\right] d t\right),
\end{aligned}
$$

from which it follows that, since both $\omega_{k}^{\prime}(\gamma) \geq 0$ for all $\gamma>0$ and $\omega_{k}(0) \geq 0$, a sufficient condition for $\Delta P \leq 0$ is

$$
\sum_{j=1}^{k}\left(\int_{\gamma}^{\gamma^{*}}\left[\phi_{j}^{F}\left(t, z_{j}\right)-\phi_{j}^{G}\left(t, z_{j}\right)\right] d t\right) \leq 0
$$

for all $k=1,2, . . n$, and for all $\gamma>0$.

Example 4 The second degree of sequential reference-based poverty dominance is checked in Fig. A2 comparing the areas under the lines $\phi_{1}\left(\gamma, z_{1}\right)$ and $\phi_{1}\left(\gamma, z_{1}\right)+$ $\phi_{2}\left(\gamma, z_{2}\right)$ for $F$ and $G$, for all $\gamma \geq 0$ as in Fig. A3 for the second stage of dominance. Given that by construction in the previous example we have first order dominance 
then the second degree dominance is implied.

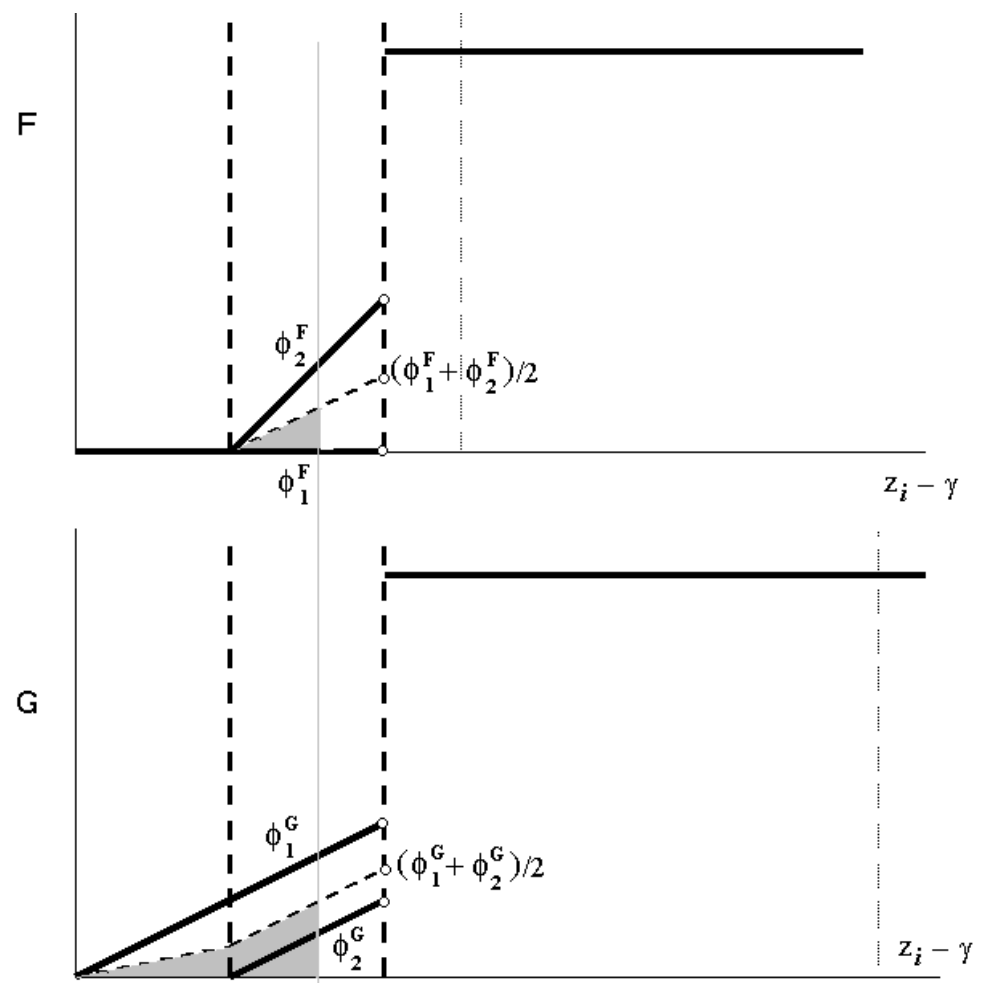

Fig A3: $2^{\text {nd }}$ order sequential reference-based dominance

Claim 7 The following conditions are equivalent:

(i) $\sum_{i=1}^{k}\left[\phi_{i}^{F}\left(\gamma, z_{i}\right)-\phi_{i}^{G}\left(\gamma, z_{i}\right)\right] \leq 0$ for all $\gamma>0$.

(ii) (a) $\gamma^{F^{(k)}}\left(p / Q_{k}^{F}, \mathbf{z}^{k}\right) \leq \gamma^{G^{(k)}}\left(p / Q_{k}^{G}, \mathbf{z}^{k}\right)$ for all $p \in\left[0, \min \left\{Q_{k}^{F}, Q_{k}^{G}\right\}\right]$

and (b) $\sum_{i=1}^{k} q_{i}^{F} \cdot H\left(F_{i}, z_{i}\right) \leq Q_{k}^{G}$, where $Q_{k}^{F}=\sum_{i=1}^{k} q_{i}^{F}$.

Proof. Consider the case of an homogeneous population, with income distribution $F(x)$, inverse distribution $F^{-1}(p)$ and poverty line $z$. The absolute poverty-gap $\gamma^{F}(p, z)$ associated with the $p^{t h}$ quantile of the income distribution is $\gamma^{F}(p, z):=$ $z-F^{*-1}(p)$ where $F^{*-1}(p)$ is the inverse of the income distribution censored at the poverty line, i.e. $F^{*-1}(p)=F^{-1}(p)$ if $p \leq F(z)$, and $F^{*-1}(p)=z$ if $p>F(z)$. Now weight the distribution using a coefficient $Q \in(0,1)$, constructing a distribution $\tilde{F}(x)$ such that

$$
\tilde{F}(x)=\left\{\begin{array}{ll}
Q \cdot F(x) & \text { if } x<z \\
Q \cdot F(x)+(1-Q) & \text { if } x \geq z
\end{array} .\right.
$$

The absolute poverty-gap for distribution $\tilde{F}$ becomes $\gamma^{\tilde{F}}(p, z):=z-\tilde{F}^{*-1}(p)$ where $\tilde{F}^{*-1}(p)=F^{*-1}(p / Q)$ if $p \leq Q$ and $\tilde{F}^{*-1}(p)=z$ if $p \in(Q, 1]$. It follows that $\gamma^{\tilde{F}}(p, z)=z-F^{*-1}(p / Q)=\gamma^{F}(p / Q, z)$ if $p \leq Q$ and $\gamma^{\tilde{F}}(p, z)=0$ if $p \in(Q, 1]$.

Consider two distributions $F, G \in \mathcal{F}$ and the associated transformations $\tilde{F}, \tilde{G}$ obtained from (26) making use of possibly different coefficients $Q^{F}$ and $Q^{G}$. It is 
well known, that $\tilde{F}$ dominates $\tilde{G}$ according to first degree stochastic dominance iff $\tilde{F}$ dominates $\tilde{G}$ in terms of rank-ordered poverty gaps i.e. $\gamma^{\tilde{F}}(p, z) \leq \gamma^{\tilde{G}}(p, z)$ for all $p \in[0,1] .{ }^{11}$

Note that condition (i) is precisely first degree stochastic dominance between $\tilde{F}$ and $\tilde{G}$ obtained from (26) using $Q_{k}^{F}$ and $F^{(k)}\left(t, \mathbf{z}^{k}\right)$ for $\tilde{F}$ and $Q_{k}^{G}$ and $G^{(k)}\left(t, \mathbf{z}^{k}\right)$ for $\tilde{G}$.

Combining the previous considerations, condition (i) is therefore equivalent to $\gamma^{\tilde{F}^{(k)}}\left(p, \mathbf{z}^{k}\right) \leq \gamma^{\tilde{G}^{(k)}}\left(p, \mathbf{z}^{k}\right)$ for all $p \in[0,1]$ where

$$
\begin{aligned}
& \gamma^{\tilde{F}^{(k)}}\left(p, \mathbf{z}^{k}\right)= \begin{cases}\gamma^{F^{(k)}}\left(p / Q_{k}^{F}, \mathbf{z}^{k}\right) & \text { if } p \in\left[0, Q_{k}^{F}\right] \\
0 & \text { if } p \in\left(Q_{k}^{F}, 1\right]\end{cases} \\
& \gamma^{\tilde{G}^{(k)}}\left(p, \mathbf{z}^{k}\right)= \begin{cases}\gamma^{G^{(k)}}\left(p / Q_{k}^{G}, \mathbf{z}^{k}\right) & \text { if } p \in\left[0, Q_{k}^{G}\right] \\
0 & \text { if } p \in\left(Q_{k}^{G}, 1\right]\end{cases}
\end{aligned}
$$

If $Q_{k}^{F}=Q_{k}^{G}$ then we clearly get condition (iia).

If $Q_{k}^{F}<Q_{k}^{G}$ then for $p=Q_{k}^{F}$ the condition is $\gamma^{F^{(k)}}\left(1, \mathbf{z}^{k}\right) \leq \gamma^{G^{(k)}}\left(Q_{k}^{F} / Q_{k}^{G}, \mathbf{z}^{k}\right)$. Condition (iia) clearly is equivalent to $\gamma^{\tilde{F}^{(k)}}\left(p, \mathbf{z}^{k}\right) \leq \gamma^{\tilde{G}^{(k)}}\left(p, \mathbf{z}^{k}\right)$ for all $p \in[0,1]$ given that $\gamma^{\tilde{F}^{(k)}}\left(p, \mathbf{z}^{k}\right)=0$ for all $p>Q_{k}^{F}$.

If $Q_{k}^{F}>Q_{k}^{G}$ then for $p=Q_{k}^{G}$ the condition is $\gamma^{F^{(k)}}\left(Q_{k}^{G} / Q_{k}^{F}, \mathbf{z}^{k}\right) \leq \gamma^{G^{(k)}}\left(1, \mathbf{z}^{k}\right)$, then given that $\gamma^{\tilde{G}^{(k)}}\left(p, \mathbf{z}^{k}\right)=0$ for all $p>Q_{k}^{G}$, in addition of (iia) it has to be also that $\gamma^{F^{(k)}}\left(p, \mathbf{z}^{k}\right)=0$ for all $p>Q_{k}^{G} / Q_{k}^{F}$ in order to guarantee $\gamma^{\tilde{F}^{(k)}}\left(p, \mathbf{z}^{k}\right) \leq \gamma^{\tilde{G}^{(k)}}\left(p, \mathbf{z}^{k}\right)$ for all $p \in[0,1]$.

That is the proportion of poor individuals in the first $k$ groups should be at most $Q_{k}^{G} / Q_{k}^{F}$. Formally $\frac{\sum_{i=1}^{k} q_{i}^{F} H\left(F_{i}, z_{i}\right)}{\sum_{i=1}^{k} q_{i}^{F}} \leq \frac{Q_{k}^{G}}{Q_{k}^{F}}$ that is $\sum_{i=1}^{k} q_{i}^{F} \cdot H\left(F_{i}, z_{i}\right) \leq Q_{k}^{G}$ as in (iib). Clearly if $Q_{k}^{F} \leq Q_{k}^{G}$ this condition is always satisfied.

Lemma $1 \int_{0}^{x}\left[F_{i}(t)-G_{i}(t)\right] d t \leq 0$ for all $x \in\left[0, z_{i}\right]$ if and only if $\int_{0}^{p}\left[\gamma^{F_{i}}\left(t, z_{i}\right)-\right.$ $\left.\gamma^{G_{i}}\left(t, z_{i}\right)\right] d t \leq 0$ for all $p \in[0,1]$.

Proof. The condition $\int_{0}^{x}\left[F_{i}(x)-G_{i}(x)\right] d x \leq 0$ for all $x \in\left[0, z_{i}\right]$ corresponds to second degree stochastic dominance of $F_{i}$ over $G_{i}$. Let $F_{i}^{-1}(p)$ be the left continuous inverse distribution function of $F_{i}(x)$. Consider the distributions of incomes $x^{*}$ that are censored at the poverty line $z_{i}$ (i.e. $x^{*}=x$ if $x<z_{i}$ and $x^{*}=z_{i}$ if $x \geq$ $\left.z_{i}\right)$. For the censored income distributions, generalized Lorenz dominance requires $\int_{0}^{p}\left[F_{i}^{-1}(t)-G_{i}^{-1}(t)\right] d t=\int_{0}^{p}\left(\left[z_{i}-G_{i}^{-1}(t)\right]-\left[z_{i}-F_{i}^{-1}(t)\right]\right) d t \geq 0$ for all $p \in[0,1]$. This is equivalent to $\int_{0}^{p}\left[\gamma^{F_{i}}\left(t, z_{i}\right)-\gamma^{G_{i}}\left(t, z_{i}\right)\right] d t \leq 0$ for all $p \in[0,1]$. The result is obtained recalling that second degree stochastic dominance is equivalent to generalized Lorenz dominance (see Atkinson, 1970, and Thistle, 1989).

\footnotetext{
${ }^{11}$ This is a trivial implication of the equivalence between first degree stochastic dominance and first degree inverse stochastic dominance or rank-dominance (Saposnik, 1981).
} 
Claim 8 The following conditions are equivalent:

(i) $\sum_{i=1}^{k}\left(\int_{\gamma}^{\gamma^{*}}\left[\phi_{i}^{F}\left(t, z_{i}\right)-\phi_{i}^{G}\left(t, z_{i}\right)\right] d t\right) \leq 0$ for all $\gamma>0$.

(ii) $Q_{k}^{F} \cdot \mathcal{P} \mathcal{G}_{F}^{k}\left(p / Q_{k}^{F}\right) \leq Q_{k}^{G} \cdot \mathcal{P} \mathcal{G}_{G}^{k}\left(p / Q_{k}^{G}\right)$ for all $p \in\left[0, \min \left\{Q_{k}^{F}, Q_{k}^{G}\right\}\right]$ and $\sum_{i=1}^{k} q_{i}^{F} H\left(F_{i}, z_{i}\right) \cdot I\left(F_{i}, z_{i}\right) \leq \sum_{i=1}^{k} q_{i}^{G} H\left(G_{i}, z_{i}\right) \cdot I\left(G_{i}, z_{i}\right)$, where $Q_{k}^{F}=\sum_{i=1}^{k} q_{i}^{F}$.

Proof. The second condition requires a clarification. Consider the case of an homogeneous population, with income distribution $F(x)$, inverse distribution $F^{-1}(p)$ and poverty line $z$. The APGP curve is $\mathcal{P} \mathcal{G}_{F}(p)=\int_{0}^{p}\left[z-F^{*-1}(t)\right] d t$ where $F^{*-1}(t)$ is the inverse of the income distribution censored at the poverty line, i.e. $F^{*-1}(p)=F^{-1}(p)$ if $p \leq F(z)$, and $F^{*-1}(p)=z$ if $p>F(z)$. Now weight the distribution using a coefficient $Q \in(0,1)$, constructing a distribution $\tilde{F}(x)$ as in $(26)$

The APGP curve for distribution $\tilde{F}$ becomes $\mathcal{P} \mathcal{G}_{\tilde{F}}(p)=\int_{0}^{p}\left[z-\tilde{F}^{*-1}(t)\right] d t$ where $\tilde{F}^{*-1}(t)=F^{*-1}(t / Q)$ if $t \leq Q$ and $\tilde{F}^{*-1}(t)=z$ if $t \in(Q, 1]$. It follows that $\mathcal{P G}_{\tilde{F}}(p)=$ $\int_{0}^{p}\left[z-F^{*-1}(t / Q)\right] d t$ if $p \leq Q$ and $\mathcal{P G}_{\tilde{F}}(p)=\int_{0}^{Q}\left[z-F^{*-1}(t / Q)\right] d t$ if $p \in(Q, 1]$. Changing the variable to $s=p / Q$, we get $\mathcal{P G}_{\tilde{F}}(p)=Q \int_{0}^{p / Q}\left[z-F^{*-1}(s)\right] d s=Q$. $\mathcal{P G}_{F}(p / Q)$ if $p \leq Q$ and $\mathcal{P G}_{\tilde{F}}(p)=Q \int_{0}^{1}\left[z-F^{*-1}(s)\right] d s=Q \cdot \mathcal{P G}_{F}(1)=Q \cdot H(F, z)$. $I(F, z)$ if $p \in(Q, 1]$.

From Lemma 1, we know that $\tilde{F}$ dominates $\tilde{G}$ according to second degree stochastic dominance iff $\tilde{F}$ dominates $\tilde{G}$ in terms of APGP curves, where $\tilde{F}$ and $\tilde{G}$ could be obtained using different coefficients $Q^{F}$ and $Q^{G}$. Once we consider the APGP curves in our approach, we face distributions of incomes (possibly of differently ranked groups) that are normalized by $Q_{k}^{F}$ and $Q_{k}^{G}$ at the generic stage $k$. The equivalence with APGP dominance is retained. Given that the procedure in which stochastic dominance is constructed does not affect the poverty gaps, we can compare $\mathcal{P} \mathcal{G}_{\tilde{F}}(p)$ and $\mathcal{P G}_{\tilde{G}}(p)$ which turn out to be equivalent to the curves $Q \cdot \mathcal{P} \mathcal{G}_{F}(p / Q)$ and similarly for $G$. In order to check that $\mathcal{P} \mathcal{G}_{\tilde{F}}(p) \leq \mathcal{P G}_{\tilde{G}}(p)$ for all $p \in[0,1]$ it is then sufficient to check that $Q_{k}^{F} \cdot \mathcal{P} \mathcal{G}_{F}^{k}\left(p / Q_{k}^{F}\right) \leq Q_{k}^{G} \cdot \mathcal{P} \mathcal{G}_{G}^{k}\left(p / Q_{k}^{G}\right)$ for all $p \in\left[0, \min \left\{Q_{k}^{F}, Q_{k}^{G}\right\}\right]$ and that $\mathcal{P} \mathcal{G}_{\tilde{F}}(1) \leq \mathcal{P} \mathcal{G}_{\tilde{G}}(1)$.

Suppose that $Q_{k}^{F} \geq Q_{k}^{G}$; then $p \in\left[0, Q_{k}^{G}\right]$. It follows with $p=Q_{k}^{G}$ that $Q_{k}^{F}$. $\mathcal{P} \mathcal{G}_{F}^{k}\left(Q_{k}^{G} / Q_{k}^{F}\right) \leq Q_{k}^{G} \cdot \mathcal{P} \mathcal{G}_{G}^{k}(1)$. While for distribution $G$ we have considered the entire $A P G P$ curve, the associated $A P G P$ curve for distribution $\tilde{G}$ is not yet completed: we are left with a horizontal part since by construction the remaining poverty gaps are zero. Thus for $p>Q_{k}^{G}$ the curve $\mathcal{P} \mathcal{G}_{F}^{k}\left(p / Q_{k}^{F}\right)$ is increasing until the point where $p=Q_{k}^{F}$. Since the curve for distribution $\tilde{G}$ reach its maximum at $Q_{k}^{G} \cdot \mathcal{P} \mathcal{G}_{G}^{k}(1)$ it will be sufficient to check that $Q_{k}^{F} \cdot \mathcal{P} \mathcal{G}_{F}^{k}(1) \leq Q_{k}^{G} \cdot \mathcal{P} \mathcal{G}_{G}^{k}(1)$ in order to guarantee the dominance for $p \in\left(Q_{k}^{G}, Q_{k}^{F}\right]$. Note that $Q_{k}^{F} \cdot \mathcal{P} \mathcal{G}_{F}^{k}(1)=\sum_{i=1}^{k} q_{i}^{F} H\left(F_{i}, z_{i}\right) \cdot I\left(F_{i}, z_{i}\right)$ as in condition (ii).

When $Q_{k}^{F}<Q_{k}^{G}$, dominance for all $p \in\left[0, Q_{k}^{F}\right]$ will be sufficient for $\mathcal{P G}_{\tilde{F}}(p) \leq$ $\mathcal{P G}_{\tilde{G}}(p)$ for all $p \in[0,1]$. In this case, the second condition in (ii) will also be implied: for $p=Q_{k}^{F}$, we will have $Q_{k}^{F} \cdot \mathcal{P} \mathcal{G}_{F}^{k}(1) \leq Q_{k}^{G} \cdot \mathcal{P} \mathcal{G}_{G}^{k}\left(Q_{k}^{F} / Q_{k}^{G}\right) \leq Q_{k}^{G} \cdot \mathcal{P} \mathcal{G}_{G}^{k}(1)$. 
Claim 9 Let $n=2 . P(F) \leq P(G)$ for all $u_{i}$ satisfying $A, A 1$ and A2 and for all $\mathbf{z} \in Z^{2}\left(\mathbf{z}^{-}, \mathbf{z}^{+}\right)$if and only if:

(i) $\int_{\gamma}^{z_{1}^{+}} \Delta \phi_{1}\left(t, z_{1}^{+}\right) d t \leq 0$ for all $\gamma>0$, and and

(iia) $\int_{\gamma}^{z_{1}^{+}} \Delta \phi_{1}\left(t, z_{1}^{+}\right) d t+\int_{\gamma}^{z_{1}^{+}} \Delta \phi_{2}\left(t, z_{2}\right) d t \leq 0$ for all $\gamma>0$ and all $z_{2} \in\left[z_{2}^{-}, z_{2}^{+}\right]$,

(iib) $\int_{\gamma}^{z_{1}} \Delta \phi_{1}\left(t, z_{1}\right) d t+\int_{\gamma}^{z_{1}} \Delta \phi_{2}\left(t, z_{2}^{+}\right) d t \leq 0$ for all $\gamma>0$ and all $z_{1} \in\left[\min \left\{z_{1}^{-}, z_{2}^{+}\right\}, z_{1}^{+}\right]$.

Proof. Recall Remark 1 in the main text. For $k=1$ we get condition (i). Let $d_{1,2}^{+}:=z_{1}^{+}-z_{2}^{+} \geq 0$ and $d_{1,2}^{-}:=\min \left\{0, z_{1}^{-}-z_{2}^{+}\right\} \geq 0$, clearly $d_{1,2}^{+} \geq d_{1,2}^{-}$. For $k=2$, if condition (iia) is satisfied then $\sum_{i=1}^{2} \int_{\gamma}^{\gamma^{*}} \Delta \phi_{i}\left(t, z_{i}\right) d t \leq 0$ for all $z_{1} \geq z_{2}$ such that $z_{1}-z_{2} \geq d_{1,2}^{+} \geq 0$. If condition (iib) is satisfied, $\sum_{i=1}^{2} \int_{\gamma}^{\gamma^{*}} \Delta \phi_{i}\left(t, z_{i}\right) d t \leq 0$ for all $z_{1} \geq z_{2}$ such that $d_{1,2}^{-} \leq z_{1}-z_{2} \leq d_{1,2}^{+}$. Note that it is impossible to have poverty lines such that $z_{1} \geq z_{2}$ and $z_{1}-z_{2}<d_{1,2}^{-}$. This completes all possible vectors $\mathbf{z} \in Z^{2}\left(\mathbf{z}^{-}, \mathbf{z}^{+}\right)$. Therefore conditions (i), (iia) and (iib) are sufficient for poverty dominance. They are also necessary, since they are associated with vectors of poverty lines in $Z^{2}\left(\mathbf{z}^{-}, \mathbf{z}^{+}\right)$.

Claim 10 Let $n=2$, and $j=1,2 . F \succcurlyeq_{S P G D(j)} G$ for all $\mathbf{z} \in Z^{n}\left(\mathbf{z}^{-}, \mathbf{z}^{+}\right)$if and only if:

(i) $\Delta_{1}^{[j]}(x) \leq 0$ for all $x \in\left[0, z_{1}^{+}\right)$;

(ii) $\Delta_{2}^{[j]}(x)+\hat{\Delta}_{1}^{[j]}\left(x ; \mathbf{z}^{-}, \mathbf{z}^{+}\right) \leq 0$ for all $x \in\left[0, z_{2}^{+}\right)$.

Proof. We follow the results in Propositions 1, that we have proved to be equivalent to $\operatorname{SPGD}(1)$ and $\operatorname{SPGD}(2)$, and construct the algorithm starting from stage 1 of the sequential comparisons. First note that letting $\gamma=z_{i}-x$ for $x \in\left[0, z_{i}\right)$ and rearranging $\Delta_{i}^{[1]}(x):=q_{i}^{F} F_{i}(x)-q_{i}^{G} G_{i}(x)$ we get $\Delta_{i}^{[1]}(x)=\Delta \phi_{i}\left(z_{i}-x, z_{i}\right)=\phi_{i}^{F}\left(\gamma, z_{i}\right)-$ $\phi_{i}^{G}\left(\gamma, z_{i}\right)$. Consequently $\Delta_{i}^{[2]}(x):=\int_{0}^{x} \Delta_{i}^{[1]}(t) d t$ is $\Delta_{i}^{[2]}(x)=\int_{0}^{x} \Delta \phi_{i}\left(z_{i}-t, z_{i}\right) d t=$ $\int_{\gamma}^{z_{i}} \Delta \phi_{i}\left(y, z_{i}\right) d y$.

(Stage 1) The condition $\Delta_{1}^{[j]}(x) \leq 0$ for all $x \in\left[0, z_{1}^{+}\right)$is therefore equivalent to the first stage of the conditions in Propositions 1. If dominance is satisfied for all $x \in\left[0, z_{1}^{+}\right)$clearly it is also satisfied for all values of $z_{1} \in\left[z_{1}^{-}, z_{1}^{+}\right]$.

(Stage 2) Consider the second stage where group 2 is introduced. For a given $\gamma>0$ sequential dominance requires that $\Delta_{2}^{[j]}\left(z_{2}-\gamma\right)+\Delta_{1}^{[j]}\left(z_{1}-\gamma\right) \leq 0$ where $z_{2} \leq z_{1}, z_{2} \in\left[z_{2}^{-}, z_{2}^{+}\right]$, and $z_{1} \in\left[z_{1}^{-}, z_{1}^{+}\right]$. Since $z_{2} \in\left[z_{2}^{-}, z_{2}^{+}\right]$we need to consider all $x_{2} \in\left[0, z_{2}^{+}\right)$for the income units in group 2 .

(2a) Suppose that $x_{2}<z_{2}^{-}$.

The minimum $\gamma$ consistent with the level of income $x_{2}$ in group 2 is $\gamma^{-}=z_{2}^{-}-x_{2}$ while the maximum $\gamma$ is $\gamma^{+}=z_{2}^{+}-x_{2}$. Therefore for $\gamma \in\left[\gamma^{-}, \gamma^{+}\right]$we get that the income level $x_{1}$ in group 1 associated with $x_{2}$ in group 2 is $x_{1}=z_{1}-\gamma \in$ $\left[z_{1}-\left(z_{2}^{+}-x_{2}\right), z_{1}-\left(z_{2}^{-}-x_{2}\right)\right]$ that is $x_{1} \in\left[x_{2}+z_{1}-z_{2}^{+}, x_{2}+z_{1}-z_{2}^{-}\right]$. Given that $z_{1} \in\left[z_{1}^{-}, z_{1}^{+}\right]$and $z_{1} \geq z_{2}$ we get that $x_{1} \in\left[x_{2}+\max \left\{0 ; z_{1}^{-}-z_{2}^{+}\right\}, x_{2}+z_{1}^{+}-z_{2}^{-}\right]$. 
(2b) Suppose that $x_{2} \in\left[z_{2}^{-}, z_{2}^{+}\right)$.

The range of poverty gaps consistent with $x_{2}$ is $\gamma \in\left(0, z_{2}^{+}-x_{2}\right]$. The income level $x_{1}$ in group 1 associated with $x_{2}$ in group 2 is $x_{1}=z_{1}-\gamma \in\left[z_{1}-\left(z_{2}^{+}-x_{2}\right), z_{1}\right)$. Given that $z_{1} \in\left[z_{1}^{-}, z_{1}^{+}\right]$and $z_{1} \geq z_{2}$ we get that $x_{1} \in\left[x_{2}+\max \left\{0 ; z_{1}^{-}-z_{2}^{+}\right\}, z_{1}^{+}\right)$.

To summarize: to each $x_{2} \in\left[0, z_{2}^{+}\right)$is associated the set of values for $x_{1}$

$$
x_{1} \in\left[x_{2}+\max \left\{0 ; z_{1}^{-}-z_{2}^{+}\right\} ; z_{1}^{+}+\min \left\{0 ; x_{2}-z_{2}^{-}\right\}\right], x_{1} \neq z_{1}^{+} .
$$

It follows that at the stage 2 of the dominance conditions it should be $\Delta_{2}^{[j]}\left(x_{2}\right)+$ $\Delta_{1}^{[j]}\left(x_{1}\right) \leq 0$ for all $x_{2} \in\left[0, z_{2}^{+}\right)$, and for all $x_{1}$ in $(27)$. For each $x_{2} \in\left[0, z_{2}^{+}\right)$we select the maximum value of $\Delta_{1}^{[j]}\left(x_{1}\right)$ for all $x_{1}$ in $(27)$. That is

$$
\hat{\Delta}_{1}^{[j]}\left(x ; \mathbf{z}^{-}, \mathbf{z}^{+}\right):=\max _{t \in\left[x+\max \left\{0 ; z_{1}^{-}-z_{2}^{+}\right\} ; z_{1}^{+}+\min \left\{0 ; x-z_{2}^{-}\right\}\right], t \neq z_{1}^{+}}\left\{\Delta_{1}^{[j]}(t)\right\} .
$$

Thus the dominance condition can be equivalently stated as

$$
\Delta_{2}^{[j]}(x)+\hat{\Delta}_{1}^{[j]}\left(x ; \mathbf{z}^{-}, \mathbf{z}^{+}\right) \leq 0 \text { for all } x \in\left[0, z_{2}^{+}\right) .
$$

Claim 11 Let $j=1,2$, and $z_{i}^{-}=z^{-} \geq 0$ for all $i=1,2, . . n$. The following statements are equivalent:

(i) $F \succcurlyeq_{S P G D(j)} G$ for all $\mathbf{z} \in Z^{n}\left(z^{-} \mathbf{1}, \mathbf{z}^{+}\right)$,

(ii) $\tilde{\Delta}_{k, k}^{[j]}\left(x_{k}, x_{k}\right) \leq 0$ for all $x_{k} \in\left[0, z_{k}^{+}\right]$and for all $k=1,2, . . n$.

Where $\tilde{\Delta}_{k, k}^{[j]}\left(x_{k}, x_{k}\right)$ is derived recursively from (30) and (31).

Proof. We follow the proof of the previous claim, and consider the third stage where group 3 is merged with the first 2 groups. For any $\gamma>0$ sequential dominance requires that $\sum_{i=1}^{3} \Delta_{i}^{[j]}\left(z_{i}-\gamma\right) \leq 0$. We consider all $x_{3} \in\left[0, z_{3}^{+}\right)$. Dominance for all $\gamma \geq z_{3}^{+}$has been satisfied already in the previous 2 stages (see previous proof).

We follow the same procedure presented for the second stage in the previous proof, and note that given $z_{i}^{-}=z^{-}$for all $i$, then $\max \left\{0 ; z^{-}-z_{3}^{+}\right\}=0$. For any $x_{3} \in\left[0, z_{3}^{+}\right)$we derive the associated values of $x_{2}$ in group 2 and $x_{1}$ in group 1 . They are, for $i=1,2$ :

$$
x_{i} \in\left[x_{3} ; z_{i}^{+}+\min \left\{0 ; x_{3}-z^{-}\right\}\right], x_{i} \neq z_{i}^{+}
$$

with associated functions

$$
\hat{\Delta}_{i}^{[j]}\left(x ; \mathbf{z}^{-}, \mathbf{z}^{+}\right):=\max _{t \in\left[x ; z_{i}^{+}+\min \left\{0 ; x-z^{-}\right\}\right], t \neq z_{i}^{+}}\left\{\Delta_{i}^{[j]}(t)\right\} .
$$

For any $x_{3} \in\left[0, z_{3}^{+}\right)$we identify the set of poverty gaps that are consistent with it. Then, for any admissible value of $x_{2}$ associated with $x_{3}$, i.e. derived by the same 
poverty gap once applied to an appropriate poverty line $z_{2}$, we identify the set of associated values of $x_{1}$.

Case 1: Let $x_{3}<z^{-}$, then $z_{3}-x_{3}=\gamma \in\left[z^{-}-x_{3}, z_{3}^{+}-x_{3}\right]$

Fix $x_{2} \in\left[x_{3} ; x_{3}+z_{2}^{+}-z^{-}\right]$from (28).

(1a) We illustrate the construction of the generic algorithm starting from $x_{2}=x_{3}$.

If $\gamma=z^{-}-x_{3}$, then $z_{2}=z^{-}$. While the minimum $x_{1}$ associated with $\gamma=z^{-}-x_{3}$ is $x_{1}=x_{3}$, and the maximum $x_{1}$ is $x_{1}=z_{1}^{+}-\left(z^{-}-x_{3}\right)=x_{3}+z_{1}^{+}-z^{-}$.

Therefore $\Delta_{3}^{[j]}\left(x_{3}\right)$ is associated with $\Delta_{2}^{[j]}\left(x_{3}\right)$ and to these are associated all the $\Delta_{1}^{[j]}(t)$ for $t \in\left[x_{3} ; z_{1}^{+}-\left(z^{-}-x_{3}\right)\right]$.

Let $z_{3}$ increase, while $x_{3}$ and $x_{2}$ are kept fixed. Then $\gamma=z_{3}-x_{3}$, and $z_{2}=z_{3}$ satisfying $z_{2} \geq z_{3}$. If $z_{2}=z_{3}$ increase then the minimum $x_{1}$ associated with $\gamma=z_{3}-x_{3}$ is $x_{1}=x_{3}$, while the maximum is $x_{1}=x_{3}+z_{1}^{+}-z_{3}<x_{3}+z_{1}^{+}-z^{-}$. Clearly the interval for the values of $\Delta_{1}^{[j]}\left(x_{1}\right)$ associated with $x_{3}=x_{2}<z^{-}$is reduced. Therefore we consider $\Delta_{1}^{[j]}(t)$ for $t \in\left[x ; z_{1}^{+}-\left(z^{-}-x\right)\right]$ for $x=x_{3}=x_{2}$.

(1b) Suppose now that $x_{2} \in\left(x_{3} ; x_{3}+z_{2}^{+}-z^{-}\right]$for $x_{3}<z^{-}$.

This implies that at least $z_{2}=x_{2}+z^{-}-x_{3}$ i.e. $z_{2} \geq z_{3}+x_{2}-x_{3}$ for $z_{3} \in$ $\left[z^{-} ; \min \left\{z_{3}^{+}, z_{2}^{+}-\left(x_{2}-x_{3}\right)\right\}\right]$.

The minimum poverty gap giving this value of $x_{2}$ is given by $\gamma=z^{-}-x_{3}$ while the maximum is given by $\min \left\{z_{2}^{+}-x_{2}, z_{3}^{+}-x_{3}\right\}$. Thus $z_{2}=x_{2}+z^{-}-x_{3}$ if $\gamma=z^{-}-x_{3}$ at one extreme and $z_{2}=x_{2}+\min \left\{z_{2}^{+}-x_{2}, z_{3}^{+}-x_{3}\right\}=\min \left\{z_{2}^{+}, z_{3}^{+}+x_{2}-x_{3}\right\}$ at the other extreme.

In the first case we set $z_{1} \geq x_{2}+z^{-}-x_{3}=z_{2}$ and identify $x_{1}=z_{1}-\gamma=$ $z_{1}-\left(z^{-}-x_{3}\right)$ for all $z_{1} \in\left[x_{2}+z^{-}-x_{3}, z_{1}^{+}\right]$. We get $x_{1} \in\left[x_{2}, z_{1}^{+}-\left(z^{-}-x_{3}\right)\right]$. This is the largest set of values for $x_{1}$ associated with $x_{2}$ given that for values of $\gamma$ larger than $z^{-}-x_{3}$ the upper bound of the interval is reduced.

As a result for any $x_{2} \in\left[x_{3} ; x_{3}+z_{2}^{+}-z^{-}\right]$we have that $x_{1} \in\left[x_{2}, z_{1}^{+}-\left(z^{-}-x_{3}\right)\right]$ if $z^{-}-x_{3}>0$.

Case 2: Let $x_{3} \in\left[z^{-}, z_{3}^{+}\right]$, then $z_{3}-x_{3}=\gamma \in\left(0, z_{3}^{+}-x_{3}\right]$

Fix $x_{2} \in\left[x_{3} ; z_{2}^{+}\right)$from $(28)$.

(2a) We start from $x_{2}=x_{3}$. If $\gamma=z_{3}-x_{3}$, for $z_{3} \in\left(x_{3}, z_{3}^{+}\right]$then $z_{2}=z_{3}$. While the minimum $x_{1}$ associated with $\gamma=z_{3}-x_{3}$ is $x_{1}=x_{3}$ (obtained setting $z_{1}=z_{2}=z_{3}$ ), and the maximum $x_{1}$ is $x_{1}=z_{1}^{+}-\left(z_{3}-x_{3}\right)=x_{3}+z_{1}^{+}-z_{3}$ (obtained setting $z_{1}=z_{1}^{+}$). Letting $z_{3} \rightarrow x_{3}$ we get $x_{1} \rightarrow z_{1}^{+}$.

Therefore for $x=x_{3}=x_{2}$ we consider all $\Delta_{1}^{[j]}(t)$ for $t \in\left[x ; z_{1}^{+}\right)$.

(2b) Suppose now that $x_{2} \in\left(x_{3} ; z_{2}^{+}\right)$for $x_{3} \in\left[z^{-}, z_{3}^{+}\right]$.

The poverty gap values $\gamma$ that are consistent with $x_{2}$ are given by $\gamma \in\left(0, \min \left\{z_{2}^{+}-\right.\right.$ $\left.\left.x_{2}, z_{3}^{+}-x_{3}\right\}\right]$. Thus the associated values of $z_{2}$ are given by $x_{2}+\gamma=z_{2} \in\left(x_{2} ; \min \left\{z_{2}^{+}, z_{3}^{+}+\right.\right.$ $\left.x_{2}-x_{3}\right\}$.

We set $z_{1} \geq z_{2}$ and identify $x_{1}=z_{1}-\gamma=z_{1}-z_{2}+x_{2}$ for all $z_{1} \in\left(x_{2} ; z_{1}^{+}\right]$. For $z_{1}=z_{2}$ we get $x_{1}=x_{2}$, as $z_{1}$ increases also $x_{1}$ increases, reaching the upper bound $x_{1}=z_{1}^{+}-z_{2}+x_{2}$. Letting $z_{2} \rightarrow x_{2}$ we get $x_{1} \in\left[x_{2} ; z_{1}^{+}\right)$as in part (2a). 
To summarize:

For any $x_{3} \in\left[0, z_{3}^{+}\right]$we get $x_{2} \in\left[x_{3} ; z_{2}^{+}+\min \left\{0 ; x_{3}-z^{-}\right\}\right], x_{2} \neq z_{2}^{+}$, and for any $x_{2}$ in this interval, we have that

$$
x_{1} \in\left[x_{2} ; z_{1}^{+}+\min \left\{0 ; x_{3}-z^{-}\right\}\right], x_{1} \neq z_{1}^{+} .
$$

More generally, starting from $x_{k} \in\left[0, z_{k}^{+}\right)$we get for $i<k$ a sequence of intervals for $x_{i}$ given by

$$
x_{i} \in\left[x_{i+1} ; z_{i}^{+}+\min \left\{0 ; x_{k}-z^{-}\right\}\right], x_{i} \neq z_{i}^{+} \text {for } i=1,2, . . k-1 .
$$

For $k>i$ we construct the algorithm letting

$$
\tilde{\Delta}_{i+1, k}^{[j]}\left(x_{i+1}, x_{k} ; \mathbf{z}^{-}, \mathbf{z}^{+}\right):=\Delta_{i+1}^{[j]}\left(x_{i+1}\right)+\hat{\Delta}_{i, k}^{[j]}\left(x_{i+1}, x_{k} ; \mathbf{z}^{-}, \mathbf{z}^{+}\right)
$$

for all $x_{i+1} \in\left[0, z_{i+1}^{+}\right]$, where $\hat{\Delta}_{0, k}^{[j]}\left(x, x_{k} ; \mathbf{z}^{-}, \mathbf{z}^{+}\right)=0$ for all $x$, while for $i=1,2, . . k-1$

$$
\hat{\Delta}_{i, k}^{[j]}\left(x, x_{k} ; \mathbf{z}^{-}, \mathbf{z}^{+}\right):=\max _{t \in\left[x ; z_{i}^{+}+\min \left\{0 ; x_{k}-z^{-}\right\}\right], t \neq z_{i}^{+}}\left\{\tilde{\Delta}_{i, k}^{[j]}\left(t, x_{k} ; \mathbf{z}^{-}, \mathbf{z}^{+}\right)\right\} .
$$

At the stage $k$ of the sequential dominance we need to compare for all $x_{k} \in\left[0, z_{k}^{+}\right]$ the sum of the values of $\Delta_{k}^{[j]}\left(x_{k}\right)$ and all possible combinations of $\Delta_{i}^{[j]}\left(x_{i}\right)$ for all $x_{i}$ consistent with $x_{k}$ derived in the first part of the proof.

We illustrate the procedure followed by the algorithm suppressing $\mathbf{z}^{-}, \mathbf{z}^{+}$from (30) and (31) for notational convenience. If $i=0$, we get $\tilde{\Delta}_{1, k}^{[j]}\left(x_{1}, x_{k}\right):=\Delta_{1}^{[j]}\left(x_{1}\right)$, then $\hat{\Delta}_{1, k}^{[j]}\left(x, x_{k}\right):=\max _{t \in\left[x ; z_{1}^{+}+\min \left\{0 ; x_{k}-z^{-}\right\}\right], t \neq z_{1}^{+}}\left\{\Delta_{1}^{[j]}(t)\right\}$. Making use of $\hat{\Delta}_{1, k}^{[j]}\left(x, x_{k}\right)$ we get $\tilde{\Delta}_{2, k}^{[j]}\left(x_{2}, x_{k}\right)=\Delta_{2}^{[j]}\left(x_{2}\right)+\hat{\Delta}_{1, k}^{[j]}\left(x_{2}, x_{k}\right)$. These are the maximum values that $\Delta_{2}^{[j]}\left(x_{2}\right)+\hat{\Delta}_{1}^{[j]}\left(x_{1}\right)$ can reach for all $x_{2}, x_{1}$ that are consistent with $x_{k}$. Continuing to the next step we get $\tilde{\Delta}_{3, k}^{[j]}\left(x_{3}, x_{k}\right)=\Delta_{3}^{[j]}\left(x_{3}\right)+\hat{\Delta}_{2, k}^{[j]}\left(x_{3}, x_{k}\right)$ giving the maximum values of $\Delta_{3}^{[j]}\left(x_{3}\right)+\Delta_{2}^{[j]}\left(x_{2}\right)+\hat{\Delta}_{1}^{[j]}\left(x_{1}\right)$ for all $x_{3}, x_{2}, x_{1}$ consistent with $x_{k}$. At the last stage we get $\tilde{\Delta}_{k, k}^{[j]}\left(x_{k}, x_{k}\right)$. According to the sequential dominance conditions at stage $k$ it should be that $\tilde{\Delta}_{k, k}^{[j]}\left(x_{k}, x_{k}\right) \leq 0$ for all $x_{k} \in\left[0, z_{k}^{+}\right]$. These conditions have to be satisfied at all stages $k=1,2, . . n$. Note also that the functions in (30) and (31) depend on $x_{k}$ given that it appears in (29).

Claim 12 Let $j=1,2$, and $z_{i}^{-}=0$ for all $i$. The following statements are equivalent:

(i) $F \succcurlyeq_{S P G D(j)} G$ for all $\mathbf{z} \in Z^{n}\left(\mathbf{0}, \mathbf{z}^{+}\right)$,

(ii) $\hat{\Delta}_{i}^{[j]}\left(0 ; \mathbf{0}, \mathbf{z}^{+}\right) \leq 0$ for all $i=1,2, \ldots n$.

Proof. Let $z_{i}^{-}=z^{-}=0$ for all $i$. Note that $\min \left\{0 ; x_{k}-z^{-}\right\}=0$ and apply the algorithm derived in the previous proof. From (29) we get

$$
x_{i} \in\left[x_{i+1} ; z_{i}^{+}\right) \text {for } i=1,2, . . k-1 .
$$


Note that $x_{i}$ depends only on $x_{i+1}$ and not on $x_{k}$ if $i<k-1$. The formula in (30) and (31) can be therefore simplified ruling out the role of $x_{k}$.

The new simplified algorithm is obtained letting for $i=0,1,2, . . n-1$

$$
\tilde{\Delta}_{i+1}^{[j]}\left(x ; \mathbf{z}^{-}, \mathbf{z}^{+}\right):=\Delta_{i+1}^{[j]}(x)+\hat{\Delta}_{i}^{[j]}\left(x ; \mathbf{z}^{-}, \mathbf{z}^{+}\right)
$$

for all $x \in\left[0, z_{i+1}^{+}\right]$, where $\hat{\Delta}_{0}^{[j]}\left(x ; \mathbf{z}^{-}, \mathbf{z}^{+}\right)=0$ for all $x$, while for $i=1,2, . . n$

$$
\hat{\Delta}_{i}^{[j]}\left(x ; \mathbf{z}^{-}, \mathbf{z}^{+}\right):=\max _{t \in\left[x ; z_{i}^{+}\right)}\left\{\tilde{\Delta}_{i}^{[j]}\left(t ; \mathbf{z}^{-}, \mathbf{z}^{+}\right)\right\} .
$$

The derived dominance conditions require that $\tilde{\Delta}_{i}^{[j]}\left(x ; \mathbf{z}^{-}, \mathbf{z}^{+}\right) \leq 0$ for all $x \in$ $\left[0, z_{i}^{+}\right)$and for all $i=1,2, \ldots n$, that is $\hat{\Delta}_{i}^{[j]}\left(0 ; \mathbf{z}^{-}, \mathbf{z}^{+}\right) \leq 0$ for all $i$.

Claim 13 If $q_{i}=q_{i}^{F}=q_{i}^{G}, F \succcurlyeq_{S P G D(2)} G$ for all $z_{1} \geq z_{2} \geq \ldots \geq z_{n}>0$ if and only if $F \succcurlyeq_{B D} G$.

Proof. Part 1: $F \succcurlyeq_{B D} G \Longrightarrow F \succcurlyeq_{S P G D(2)} G$ for all $z_{1} \geq z_{2} \geq \ldots \geq z_{n}>0$.

Let $q_{i}=q_{i}^{F}=q_{i}^{G}$, for a fixed vector $\mathbf{z}$ and a fixed stage $k$ of $S P G D(2)$ we have $\sum_{i=1}^{k}\left(\int_{\gamma}^{\gamma^{*}}\left[\phi_{i}^{F}\left(t, z_{i}\right)-\phi_{i}^{G}\left(t, z_{i}\right)\right] d t\right) \leq 0$ for all $\gamma>0$. Substituting for $\gamma^{*}=z_{1}$ and $\phi_{i}^{F}\left(t, z_{i}\right):=q_{i} F_{i}\left(z_{i}-t\right)$ where $F_{i}(x)=0$ for $x<0$ we get

$$
\sum_{i=1}^{k} q_{i}\left(\int_{\gamma}^{z_{1}}\left[F_{i}\left(z_{i}-t\right)-F_{i}\left(z_{i}-t\right)\right] d t\right) \leq 0
$$

for all $\gamma>0$. Letting $x_{j}=0$ for all $j>k$ in BD condition, we get

$$
\sum_{i=1}^{k} q_{i} \int_{0}^{x_{i}}\left[F_{i}(t)-G_{i}(t)\right] d t \leq 0 .
$$

For any $\gamma$ and $z_{i}$ there exists a value of $x_{i}$ such that $\int_{\gamma}^{z_{1}} F_{i}\left(z_{i}-t\right) d t=\int_{0}^{x_{i}} F_{i}(t) d t$ this is precisely the case for $x_{i}=z_{i}-\gamma$. As $\gamma$ or the vector $\mathbf{z}^{k}=\left(z_{1}, \ldots z_{i}, . . z_{k}\right)$ change there is always a vector with $x_{1} \geq x_{2} \geq \ldots \geq x_{n} \geq 0$ such that the $\operatorname{SPGD}(2)$ conditions at stage $k$ can be obtained as special case of BD conditions. Given that this consideration holds for any $k=1,2, . . n$ then Part 1 is proved.

Part 2: $F \succcurlyeq_{B D} G \Longleftarrow F \succcurlyeq_{S P G D(2)} G$ for all $z_{1} \geq z_{2} \geq \ldots \geq z_{n}>0$.

Consider BD conditions evaluated for a specific vector $x_{1}, x_{2}, . . x_{n}$ s.t. $x_{1} \geq x_{2} \geq$ $\ldots \geq x_{n} \geq 0$. For any $x_{i} \geq 0$ there exist a value of $\gamma>0$ and at least a vector of ranked poverty lines $\mathbf{z}^{n}$ such that $x_{i}=\max \left\{0, z_{i}-\gamma\right\}$ for all $i=1,2$,..n. It follows that

$$
\int_{0}^{x_{i}} F_{i}(t) d t=\int_{\gamma}^{z_{1}} F_{i}\left(z_{i}-t\right) d t
$$

and therefore the BD condition can be obtained as the last stage of an appropriate SPGD(2) condition. Given that this consideration holds for all vectors $x_{1} \geq x_{2} \geq$ $\ldots \geq x_{n} \geq 0$, we have proved Part 2 . 
Results for construction of Map A In order to prove the next claims we will make use of results by Müller (1997) and Castagnoli and Maccheroni (1998) valid for stochastic dominance conditions obtained as unanimous dominance for additively decomposable evaluation functionals. For expositional convenience we rephrase the results in the following Lemma adapting them to our framework. Let $\mathcal{U}$ denote a set of admissible deprivation functions $u_{i}$, and consider the associated dominance condition $F \succcurlyeq_{\mathcal{U}} G$ if and only if $P(F) \leq P(G)$ for all $u_{i} \in \mathcal{U}$. Moreover we will say that a deprivation function $\hat{u}_{i}$ preserves $\succcurlyeq_{\mathcal{U}}$ if $F \succcurlyeq_{\mathcal{U}} G$ implies that $P(F) \leq P(G)$ for $u_{i}=\hat{u}_{i}$.

Lemma 2 Suppose $F \succcurlyeq_{\mathcal{U}} G$ then the deprivation function $\hat{u}_{i}$ preserves $\succcurlyeq_{\mathcal{U}}$ if and only if $\hat{u}_{i}$ belongs to the closure of the convex cone generated by $\mathcal{U}$ and all constant functions.

Remark 3 Let $\mathcal{V}$ denote a set of deprivation functions whose closure is $\mathcal{V}^{*}$ and denote by $C^{*}(\mathcal{U})$ the closure of the convex cone generated by $\mathcal{U}$ and all constant functions. In order to derive dominance condition $F \succcurlyeq_{\mathcal{V}} G$ it is sufficient to identify a set of deprivation functions $\mathcal{U}$ s.t. $\mathcal{U} \subseteq \mathcal{V}^{*}$ and $\mathcal{V} \subseteq C^{*}(\mathcal{U})$. Condition $F \succcurlyeq_{\mathcal{U}} G$ will be sufficient to guarantee $F \succcurlyeq_{\mathcal{V}} G$ since $\mathcal{V} \subseteq C^{*}(\mathcal{U})$ moreover, it will be also necessary since $\mathcal{U} \subseteq \mathcal{V}^{*}$.

Claim 14 For a fixed $\mathbf{z} \in Z^{n}, P(F) \leq P(G)$ for all $u_{i}$ satisfying properties $A, A O$ and $W M$ if and only if $\hat{\Delta}_{i}^{[1]}(0 ; \mathbf{z}) \leq 0$ for all $i=1,2, \ldots n$.

Proof. Necessity: we consider a set of deprivation functions that can be obtained as limits of functions $u_{i}$ satisfying properties A, A0 and WM, thus they belong to the closure of the set of admissible functions considered. Let

$$
\begin{aligned}
& u_{i}\left(z_{i}-x\right):=\left\{\begin{array}{c}
0 \quad \text { for all } x \in\left(z_{i}-\gamma_{i}, z_{i}\right] \\
\alpha_{i}>0 \text { for all } x \in\left[0, z_{i}-\gamma_{i}\right]
\end{array}\right. \\
& \text { where } \gamma_{i} \geq \gamma_{i-1} \text { and } \alpha_{i} \leq \alpha_{i-1} \text { for all } i=2,3, . . n
\end{aligned}
$$

with $\gamma_{i} \leq z_{i}$. Poverty dominance requires that $\Delta P=\sum_{i=1}^{n} \int_{0}^{z_{i}} u_{i}\left(z_{i}-x\right) d\left[\Delta_{i}^{[1]}(x)\right] \leq$ 0 where $\Delta_{i}^{[1]}(x):=q_{i}^{F} F_{i}(x)-q_{i}^{G} G_{i}(x)$. When the functions $u_{i}\left(z_{i}-x\right)$ in (35) are considered then poverty dominance is obtained if and only if:

$$
\Delta P=\sum_{i=1}^{n} \alpha_{i} \cdot \Delta_{i}^{[1]}\left(z_{i}-\gamma_{i}\right) \leq 0
$$

for all $\alpha_{i} \leq \alpha_{i-1}$, and all $\gamma_{i} \geq \gamma_{i-1}$ where $\gamma_{i} \in\left(0, z_{i}\right]$.

Letting $\alpha_{i}=\beta>0$ for all $i=j+1, j+2, . . n$ and $\alpha_{i}=\alpha>\beta$ for all $i=1,2, . . j$ we get $\Delta P=\alpha \sum_{i=1}^{j} \Delta_{i}^{[1]}\left(z_{i}-\gamma_{i}\right)+\beta \sum_{i=j+1}^{n} \Delta_{i}^{[1]}\left(z_{i}-\gamma_{i}\right)$. Letting $\beta \rightarrow 0$ then $\Delta P \leq 0$ requires that

$$
\sum_{i=1}^{j} \Delta_{i}^{[1]}\left(z_{i}-\gamma_{i}\right) \leq 0 \text { for all } \gamma_{i} \geq \gamma_{i-1}>0 \text { for all } j=1,2, . . n .
$$


Any income level in group $i$ is compared with all (not-lower) incomes in the lower index groups associated with the same or lower poverty gap levels. For a fixed vector of ranked poverty lines $\mathbf{z} \in Z^{n}$ we can summarize the condition in (36) making use of the following algorithm: for $i=0,1,2, . . n-1$ let

$$
\tilde{\Delta}_{i+1}^{[1]}(x ; \mathbf{z}):=\Delta_{i+1}^{[1]}(x)+\hat{\Delta}_{i}^{[1]}\left(x+\delta_{i} ; \mathbf{z}\right) \text { for all } x \in\left[0, z_{i+1}\right),
$$

where $\hat{\Delta}_{0}^{[1]}(x ; \mathbf{z}):=0$ for all $x, z_{0}:=z_{1}$ and $\delta_{i}:=z_{i}-z_{i+1}$ while for $i=1,2, . . n$ we define

$$
\hat{\Delta}_{i}^{[1]}(x ; \mathbf{z}):=\max _{t \in\left[x ; z_{i}\right)}\left\{\tilde{\Delta}_{i}^{[1]}(t ; \mathbf{z})\right\} .
$$

Condition (36) requires that $\hat{\Delta}_{i}^{[1]}(0 ; \mathbf{z}) \leq 0$ for all $i=1,2, . . n$.

Sufficiency: Making use of Lemma 2 it is sufficient to point out that the set of all $u_{i}$ satisfying properties $\mathrm{A}, \mathrm{A} 0$ and WM belongs to the closure of the convex cone generated by the functions in (35). Recalling that any monotonic continuous function can be derived as the limit of a series of step functions [See Chapter 1 in Asplund and Bungart (1966)], those in (35) can lead to all poverty deprivation functions that are non-decreasing in $\gamma$ moreover by construction the deprivation functions of less needy groups are not above those of needier groups at each poverty gap level.

Claim 15 For a fixed $\mathbf{z} \in Z^{n}, P(F) \leq P(G)$ for all $u_{i}$ satisfying properties $A, A 1$ and WPT if and only if $\hat{\Delta}_{i}^{[2]}(0 ; \mathbf{z}) \leq 0$ for all $i=1,2, \ldots$.

Proof. Considering $\Delta P$ obtained in (19) we have

$$
\Delta P=\sum_{i=1}^{n} \int_{0}^{z_{i}} u_{i}^{\prime}\left(z_{i}-x\right) d\left[\Delta_{i}^{[2]}(x)\right] .
$$

where $\Delta_{i}^{[2]}(x):=\int_{0}^{x} \Delta_{i}^{[1]}(t) d t$ and $u_{i}^{\prime}$ denotes derivative w.r.t. $z_{i}-x$. Let $v_{i}(\gamma):=$ $u_{i}^{\prime}\left(z_{i}-x\right)$ where $\gamma=z_{i}-x$. Then according to A1 we have $v_{i}(\gamma) \geq v_{i+1}(\gamma) \geq 0$ for all $\gamma>0$, and all $i=1,2, . . n-1$, and property $\mathrm{A}$ is implied by $\mathrm{A} 1$ and $u_{i}(0)=0$, while WPT requires that $v_{i}(\gamma)$ is non-decreasing in $\gamma$. The basis for the class of admissible functions $v_{i}\left(z_{i}-x\right)$ is defined in (35). Considering this set of functions we can derive the necessary and sufficient conditions for unanimous dominance $\Delta P=$ $\sum_{i=1}^{n} \int_{0}^{z_{i}} v_{i}\left(z_{i}-x\right) \cdot d\left[\Delta_{i}^{[2]}(x)\right] \leq 0$ for all $v_{i}(\gamma)$ satisfying A1 and WPT just substituting $\Delta_{i}^{[2]}(x)$ for $\Delta_{i}^{[1]}(x)$ within the proof of Claim 14 .

We generalize the algorithms applied in Propositions 8 and 9 introducing a vector $\boldsymbol{\alpha}$ of parameters $\alpha_{i} \in[0,1]$ for $i=1,2, \ldots n-1$ that allow to shift at each stage $i$ of the between groups comparisons (involving groups $i$ and $i+1$ ) the focus from poverty gaps (for $\alpha_{i}=1$ ) to incomes (for $\alpha_{i}=0$ ). 
For an ordered vector of poverty lines $\mathbf{z} \in Z^{n}$ we consider $j=1,2$ and for $i=0,1,2, . . n-1$ we let

$$
\tilde{\Delta}_{i+1}^{\alpha[j]}(\boldsymbol{\alpha} ; x ; \mathbf{z}):=\Delta_{i+1}^{[j]}(x)+\hat{\Delta}_{i}^{\alpha[j]}\left(\boldsymbol{\alpha} ; x+\alpha_{i} \cdot \delta_{i} ; \mathbf{z}\right) \text { for all } x \in\left[0, z_{i+1}\right),
$$

where $\hat{\Delta}_{0}^{[j]}(\boldsymbol{\alpha} ; x ; \mathbf{z}):=0$ for all $x, z_{0}:=z_{1}, \alpha_{0}:=\alpha_{1}$ and $\delta_{i}:=z_{i}-z_{i+1}$ while for $i=1,2, . . n$ we define

$$
\hat{\Delta}_{i}^{\alpha[j]}(\boldsymbol{\alpha} ; x ; \mathbf{z}):=\max _{t \in\left[x ; z_{i}\right)}\left\{\tilde{\Delta}_{i}^{\alpha[j]}(\boldsymbol{\alpha} ; t ; \mathbf{z})\right\}
$$

Note that dominance conditions $\hat{\Delta}_{i}^{[j]}(0 ; \mathbf{z}) \leq 0$ presented in Claim 14 coincide with $\hat{\Delta}_{i}^{\alpha[j]}(\mathbf{1} ; 0 ; \mathbf{z}) \leq 0$ while $\hat{\Delta}_{i}^{[j]}\left(0 ; \mathbf{0}, \mathbf{z}^{+}\right) \leq 0$ derived in Proposition 6 coincide with $\hat{\Delta}_{i}^{\alpha[j]}\left(\mathbf{0} ; 0 ; \mathbf{z}^{+}\right) \leq 0$.

The dominance conditions based on $\hat{\Delta}_{i}^{\alpha[j]}(\mathbf{0} ; x ; \mathbf{z})$ provide the counterpart within the income approach of those derived in Propositions 8 and 9 following the poverty gap approach. Direct modifications of the proofs of Propositions 8 and 9 where income levels are substituted for poverty gap levels allow to derive the following results.

Claim 16 For a fixed $\mathbf{z} \in Z^{n}, P(F) \leq P(G)$ for all $u_{i}$ satisfying properties $A, A 0^{*}$, and $W M$ if and only if $\hat{\Delta}_{i}^{\alpha[1]}(\mathbf{0} ; x ; \mathbf{z}) \leq 0$ for all $i=1,2, \ldots n$.

Claim 17 For a fixed $\mathbf{z} \in Z^{n}, P(F) \leq P(G)$ for all $u_{i}$ satisfying properties $A, A 1^{*}$ and WPT if and only if $\hat{\Delta}_{i}^{\alpha[2]}(\mathbf{0} ; x ; \mathbf{z}) \leq 0$ for all $i=1,2, \ldots n$.

These results are essentially straightforward generalizations of the result of Bourguignon (1989).

The (horizontal) implications in Map A, can also be derived noticing that:

Remark 4 If $\alpha_{i} \geq \hat{\alpha}_{i}$ for all $i=1,2, . . n-1$ then $\tilde{\Delta}_{i}^{\alpha[j]}(\boldsymbol{\alpha} ; x ; \mathbf{z}) \leq \tilde{\Delta}_{i}^{\alpha[j]}(\hat{\boldsymbol{\alpha}} ; x ; \mathbf{z})$ for all $x \geq 0, \mathbf{z} \in Z^{n}, i=1,2,$. n. As a special case: $\tilde{\Delta}_{i}^{\alpha[j]}(\mathbf{1} ; x ; \mathbf{z}) \leq \tilde{\Delta}_{i}^{\alpha[j]}(\mathbf{0} ; x ; \mathbf{z})$.

The reason is that if $\delta_{i}>0$ then according to $(40)$ condition $\tilde{\Delta}_{i+1}^{[j]}\left(\alpha_{i+1} ; x ; \mathbf{z}\right) \leq$ $\tilde{\Delta}_{i+1}^{[j]}\left(\hat{\alpha}_{i+1} ; x ; \mathbf{z}\right)$ coincides with

$$
\max _{t \in\left[x+\alpha_{i} \cdot \delta_{i} ; z_{i}\right)}\left\{\tilde{\Delta}_{i}^{\alpha[j]}(\boldsymbol{\alpha} ; t ; \mathbf{z})\right\} \leq \max _{t \in\left[x+\hat{\alpha}_{i} \cdot \delta_{i} ; z_{i}\right)}\left\{\tilde{\Delta}_{i}^{\alpha[j]}(\hat{\boldsymbol{\alpha}} ; t ; \mathbf{z})\right\}
$$

which is always satisfied for all $i=1,2, . . n$ if $\boldsymbol{\alpha} \geq \hat{\boldsymbol{\alpha}}$ given that $x+\hat{\alpha}_{i} \cdot \delta_{i} \leq x+\alpha_{i} \cdot \delta_{i}$. 
Results for construction of Map B We move now to derive some results summarized in Map B. We extend the results on intermediate dominance requiring conditions that are robust to changes in poverty lines, both within the poverty gap framework and within the income framework.

We consider vectors of ranked poverty lines belonging to $Z^{n}\left(\mathbf{0}, \mathbf{z}^{+}\right)$. Next claim is an equivalent version of Proposition 10:

Claim 18 Let $j=1,2 . \hat{\Delta}_{i}^{\alpha[j]}(\mathbf{1} ; 0 ; \mathbf{z}) \leq 0$ for all $i=1,2, \ldots$ n and for all $\mathbf{z} \in Z^{n}\left(\mathbf{0}, \mathbf{z}^{+}\right)$ if and only if $\hat{\Delta}_{i}^{\alpha[j]}\left(\mathbf{0} ; 0 ; \mathbf{z}^{+}\right) \leq 0$ for all $i=1,2, \ldots n$.

Proof. We derive the result by induction starting from comparisons involving incomes in the first two groups and then extending the analysis to the other groups.

Consider a vector $\mathbf{z} \in Z^{n}\left(\mathbf{0}, \mathbf{z}^{+}\right)$such that $z_{1}=z_{2}$ and select an income value $x_{2}$ in group 2 s.t. $x_{2}<z_{2}$. Then, according to $\tilde{\Delta}_{2}^{\alpha[j]}(\mathbf{1} ; x ; \mathbf{z})$ in $(40)$ and $(41)$ all income levels $x_{1}$ in group 1 that are comparable to $x_{2}$ belong to the range $\left[x_{2}, z_{1}\right)$. Suppose $z_{1}^{+}>z_{2}$. If we let $z_{1}$ increase till $z_{1}^{+}$then the values for $x_{1}$ in group 1 that are comparable to $x_{2}$ belong to the range $\left[x_{2}+z_{1}-z_{2}, z_{1}\right)$. According to $\tilde{\Delta}_{2}^{\alpha[j]}(\mathbf{1} ; x ; \mathbf{z})$ the value $\Delta_{2}^{[j]}\left(x_{2}\right)$ should be added to the maximum of $\Delta_{1}^{[j]}\left(x_{1}\right)$ for all the values of $x_{1}$ within the admissible ranges $\left[x_{2}+z_{1}-z_{2}, z_{1}\right)$ for $z_{2} \leq z_{1} \leq z_{1}^{+}$. Considering the maximum over each one of these ranges will coincide with taking the maximum of $\Delta_{1}^{[j]}\left(x_{1}\right)$ for all values in $\left[x_{2}, z_{1}\right)$. Thus for each $x_{2}<z_{2} \leq z_{2}^{+}$in order to derive a dominance condition that is satisfied for all $z_{2} \leq z_{1} \leq z_{1}^{+}$it is necessary to consider $\hat{\Delta}_{1}^{\alpha[j]}\left(\mathbf{0} ; x_{2} ; \mathbf{z}^{+}\right)$leading to the condition $\hat{\Delta}_{2}^{\alpha[j]}\left(\mathbf{0} ; 0 ; \mathbf{z}^{+}\right) \leq 0$.

Consider the dominance at the third stage. For each value $x_{3}<z_{3}$ in group 3 and for a fixed $z_{3} \leq z_{3}^{+}$we need to add to $\Delta_{3}^{[j]}\left(x_{3}\right)$ the maximum of $\Delta_{2}^{[j]}\left(x_{2}\right)+$ $\hat{\Delta}_{1}^{\alpha[j]}\left(\mathbf{0} ; x_{2} ; \mathbf{z}^{+}\right)$for all $x_{2} \in\left[x_{3}+z_{2}-z_{3}, z_{2}\right)$. Since the condition should hold for all $z_{3} \in\left[z_{2} ; z_{3}^{+}\right]$then we will have a set of ranges for the values of $x_{2}$ that are comparable to $x_{3}$. The maximum of $\Delta_{2}^{[j]}\left(x_{2}\right)+\hat{\Delta}_{1}^{\alpha[j]}\left(\mathbf{0} ; x_{2} ; \mathbf{z}^{+}\right)$over all these ranges will coincide with the maximum evaluated over $\left[x_{3}, z_{2}\right)$ thereby leading to the definition of $\hat{\Delta}_{2}^{\alpha[j]}\left(\mathbf{0} ; x_{3} ; \mathbf{z}^{+}\right)$thus inducing the condition $\hat{\Delta}_{3}^{\alpha[j]}\left(\mathbf{0} ; 0 ; \mathbf{z}^{+}\right) \leq 0$. Similar considerations apply to comparisons involving all the remaining groups in sequence. The proof is completed noticing that $\hat{\Delta}_{1}^{\alpha[j]}\left(\mathbf{0} ; 0 ; \mathbf{z}^{+}\right)=\hat{\Delta}_{1}^{\alpha[j]}\left(\mathbf{1} ; 0 ; \mathbf{z}^{+}\right)$.

Next results allow to prove the (horizontal) equivalence relations in Map B.

Remark 5 Let $j=1,2$. If $z_{i} \leq \hat{z}_{i}$ for all $i=1,2$,..n then $\tilde{\Delta}_{i}^{\alpha[j]}(\mathbf{0} ; x ; \mathbf{z}) \leq \tilde{\Delta}_{i}^{\alpha[j]}(\mathbf{0} ; x ; \hat{\mathbf{z}})$ for all $x \in\left[0, z_{i}\right)$, all $i=1,2, . . n$.

The remark is a direct implication of the fact that according to (40) condition $\tilde{\Delta}_{i+1}^{\alpha[j]}(\mathbf{0} ; x ; \mathbf{z}) \leq \tilde{\Delta}_{i+1}^{\alpha[j]}(\mathbf{0} ; x ; \hat{\mathbf{z}})$ for all $x \in\left[0, z_{i+1}\right)$ coincides with

$$
\max _{t \in\left[x ; z_{i}\right)}\left\{\tilde{\Delta}_{i}^{\alpha[j]}(\mathbf{0} ; t ; \mathbf{z})\right\} \leq \max _{t \in\left[x ; \hat{z}_{i}\right)}\left\{\tilde{\Delta}_{i}^{\alpha[j]}(\mathbf{0} ; t ; \hat{\mathbf{z}})\right\}
$$


which is always satisfied for all $i=1,2, . . n-1$ if $z_{i} \leq \hat{z}_{i}$. Note that the statement in the remark confines the admissible range of $x$ for the comparison of functions $\tilde{\Delta}_{i}^{\alpha[j]}$ to values below the lower poverty line associated with group $i$. But the construction of functions $\tilde{\Delta}_{i}^{\alpha[j]}(\mathbf{0} ; x ; \mathbf{z})$ and $\tilde{\Delta}_{i}^{\alpha[j]}(\mathbf{0} ; x ; \hat{\mathbf{z}})$ is derived according to (40) and (41) thus the domain of $\tilde{\Delta}_{i}^{\alpha[j]}(\mathbf{0} ; x ; \hat{\mathbf{z}})$ includes also values of $x$ lying within the range $\left(z_{i}, \hat{z}_{i}\right)$.

An immediate corollary can be derived from the application of the remark.

Corollary $\mathbf{5}$ Let $j=1,2 . \hat{\Delta}_{i}^{\alpha[j]}(\mathbf{0} ; 0 ; \mathbf{z}) \leq 0$ for all $i=1,2, \ldots$ and for all $\mathbf{z} \in$ $Z^{n}\left(\mathbf{0}, \mathbf{z}^{+}\right)$if and only if $\hat{\Delta}_{i}^{\alpha[j]}\left(\mathbf{0} ; 0 ; \mathbf{z}^{+}\right) \leq 0$ for all $i=1,2, \ldots n$.

Proof. $\hat{\Delta}_{i}^{\alpha[j]}\left(\mathbf{0} ; 0 ; \mathbf{z}^{+}\right) \leq 0$ for all $i=1,2, \ldots n$ is a necessary condition. If it holds then, according to Remark 5 also for all $z_{i} \leq z_{i}^{+}$we have $\tilde{\Delta}_{i}^{\alpha[j]}(\mathbf{0} ; x ; \mathbf{z}) \leq \tilde{\Delta}_{i}^{\alpha[j]}\left(\mathbf{0} ; x ; \mathbf{z}^{+}\right) \leq 0$ for all $x \in\left[0, z_{i}\right)$, all $i=1,2, . n$. That is we obtain the sufficiency part of the statement i.e. $\hat{\Delta}_{i}^{\alpha[j]}(\mathbf{0} ; 0 ; \mathbf{z}) \leq 0$ for all $i$, and for all $\mathbf{z} \in Z^{n}\left(\mathbf{0}, \mathbf{z}^{+}\right)$.

An implication of the result is:

Corollary 6 Let $j=1,2$. The following statements are equivalent:

(i) $\hat{\Delta}_{i}^{\alpha[j]}(\mathbf{1} ; 0 ; \mathbf{z}) \leq 0$ for all $i=1,2, \ldots n$ and for all $\mathbf{z} \in Z^{n}\left(\mathbf{0}, \mathbf{z}^{+}\right)$.

(ii) $\hat{\Delta}_{i}^{\alpha[j]}(\mathbf{0} ; 0 ; \mathbf{z}) \leq 0$ for all $i=1,2, \ldots n$ and for all $\mathbf{z} \in Z^{n}\left(\mathbf{0}, \mathbf{z}^{+}\right)$.

These are the (horizontal) equivalence relations in Map B. 